\title{
The Intrusiveness of Sensor-Suit Components on the Postures Associated with Performing Repeated Whole-Body Manual Lifting Tasks
}

Lauren Amanda Visnansky

West Virginia University

Follow this and additional works at: https://researchrepository.wvu.edu/etd

\section{Recommended Citation}

Visnansky, Lauren Amanda, "The Intrusiveness of Sensor-Suit Components on the Postures Associated with Performing Repeated Whole-Body Manual Lifting Tasks" (2011). Graduate Theses, Dissertations, and Problem Reports. 4810.

https://researchrepository.wvu.edu/etd/4810

This Thesis is protected by copyright and/or related rights. It has been brought to you by the The Research Repository @ WVU with permission from the rights-holder(s). You are free to use this Thesis in any way that is permitted by the copyright and related rights legislation that applies to your use. For other uses you must obtain permission from the rights-holder(s) directly, unless additional rights are indicated by a Creative Commons license in the record and/ or on the work itself. This Thesis has been accepted for inclusion in WVU Graduate Theses, Dissertations, and Problem Reports collection by an authorized administrator of The Research Repository @ WVU. For more information, please contact researchrepository@mail.wvu.edu. 
The Intrusiveness of Sensor-Suit Components on the Postures Associated with Performing Repeated Whole-Body Manual Lifting Tasks

Lauren Amanda Visnansky

Thesis submitted to the

College of Engineering and Mineral Resources

at West Virginia University

in partial fulfillment of the requirements

for the degree of

Master of Science

in

Industrial Engineering

Kevin Rider, Ph.D., Chair

Frank Buczek, Jr., Ph.D.

Ashish Nimbarte, Ph.D.

Department of Industrial and Management Systems Engineering

Morgantown, West Virginia

2011

Keywords: Posture, Lifting, Manual Handling, Mass, Banding, Clo

Copyright 2011 Lauren A. Visnansky 


\section{ABSTRACT \\ The Intrusiveness of Sensor-Suit Components on the Postures Associated with Performing Repeated Whole-Body Manual Lifting Tasks}

\section{Lauren Amanda Visnansky}

Ergonomics can be defined as the responsibility to ensure that the demands of a job do not exceed the capabilities of a worker (Garg, Chaffin, and Herrin, 1978). Evaluating physiological demands on workers, particularly individuals performing highly varied tasks or monitoring their work in the field, can be a complex problem. Using on-person sensors to record kinematic and physiological measurements throughout extended is one proposed method by which to collect data necessary to evaluate the demands placed on the workers. In order to assess the efficacy of the data that would be collected, it is critical to evaluate the intrusive effects of the onperson sensors on the manner in which the work is performed. For the purpose of this study, various outfit ensembles, consisting of combinations of Clo, Mass, and Banding, were analyzed in order to determine whether or not they affect the posture while individuals perform a repetitive lifting task. Thirty-two paid volunteers participated in this study. Each subject was randomly assigned one of eight experimental ensembles. VICON MX-13 near-infrared cameras were used to capture whole-body kinematics. Subjects were asked to perform a cyclic work protocol that consisted of six twenty-minute cycles, including five-minutes each of 30 groundto-waist lifts and lowers, 30 standing arm lifts and lowers, continuous walking on a treadmill, and a rest period. A $2^{3}$ factorial, between-subjects design was used, producing 8 experimental ensemble conditions for combinations of Clo, Mass, and Banding. Statistical analyses were performed with a stepwise regression and general linear model (GLM). The bilateral angles for the hips, knees, and ankles were the dependent variables; independent variables were Banding, Clo, Mass, Part, Phase, and Cycle. From the stepwise regression ( $\alpha=0.10$ to remove), Part and Phase were removed from the model. From the GLM, the adjusted $R^{2}$ values indicated that $a$ good fit existed between the variables in the model. ANOVA results indicated that the main effects and all interactions effects of the ensemble conditions were significant, but the significance varied across lower body joints. Results indicate that Banding and Clo have significant effects on posture, but their effects are less than the nominally fatiguing aspect of the tasks performed. As expected, adding mass to the subjects caused significant changes to their posture over time, suggesting elevated levels of fatigue. Future studies should include other populations, fitness and experience constraints, and tasks with a lower physiological burden. 


\section{DEDICATION}

This thesis is dedicated to my grandfather, Robert Batto, and in memory of my grandmother, Mildred Batto. I could not have become the person who I am without them. 


\section{ACKNOWLEDGEMENTS}

Foremost, I would like to thank Dr. Rider, my advisor and mentor. He has been there since day one, and has gone above and beyond to help me complete my work. His continual support and encouragement have allowed me to push through some of the most difficult times, and to come out a stronger, better person. I truly would not have been able to finish my thesis without him. It has been refreshing to see a faculty member so committed to the development and success of his students.

To my committee members, Dr. Buczek and Dr. Nimbarte, of whom I owe much thanks. I appreciate the time and dedication that they gave to me so that I could accomplish my goals. I am grateful to Dr. Buczek for his patient and dedication to my research, and to Dr. Nimbarte for his willingness and selflessness to be a part of my committee.

I would like to offer special thanks to all of those individuals who have helped me along the way, and have been in full support of me while completing my thesis. Words cannot express how grateful I am for them. To Kristin Goode, an extra thank you for the time and effort that she dedicated to aiding in my research, and Erik Sinsel, for his assistance with my data collection.

Much thanks to my parents, John and Kristine Wolbert, for their never-ending support, love, and encouragement. They have played such a vital role in my life, and I cannot thank them enough for all that they have done. Everything that they have done for me was done with my best interests in mind. They have only ever wanted to see me happy, and have done everything in their power to make that happen. I could not ask for better parents.

Finally, to my husband, Andy, for being an amazing source of support and his unconditional love. He has been with me through the good and the bad, and has made even the bumpiest ride smoother. It is impossible to imagine making the journey through my graduate

work and life without him. He has been a driving force behind me in all that I have done. I am so fortunate to have someone like this by my side. 


\section{TABLE OF CONTENTS}

ABSTRACT

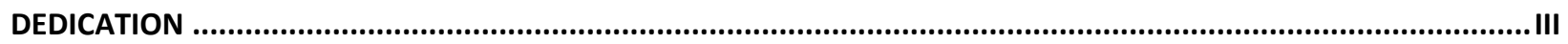

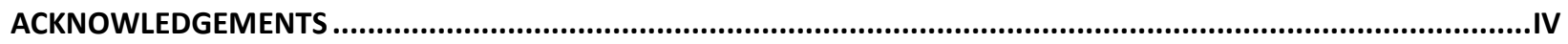

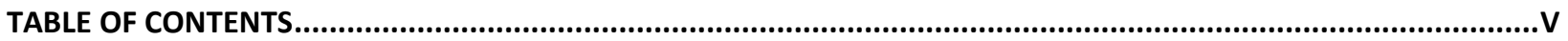

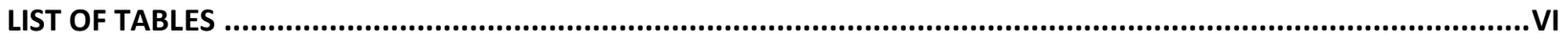

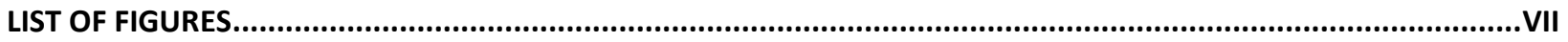

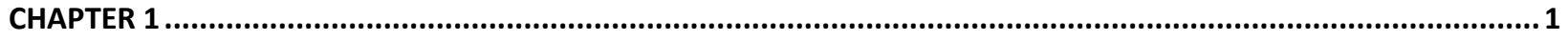

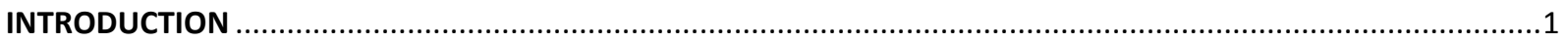

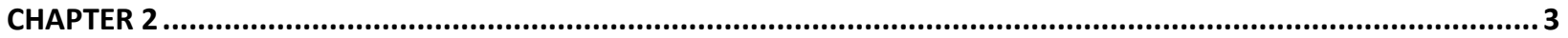

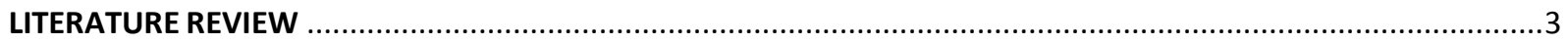

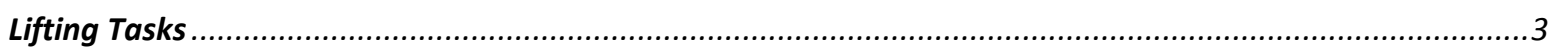

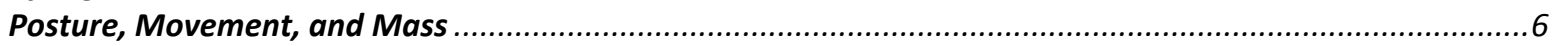

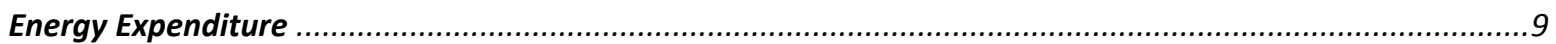

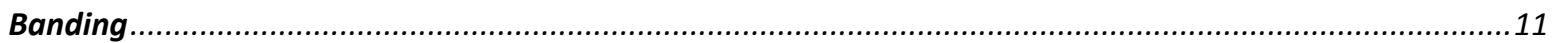

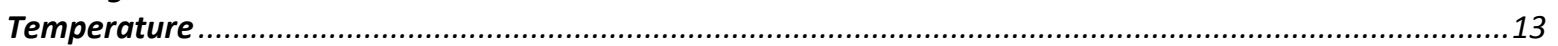

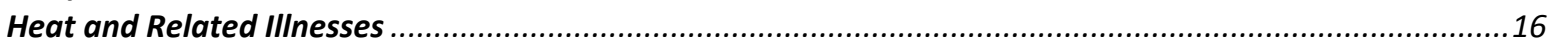

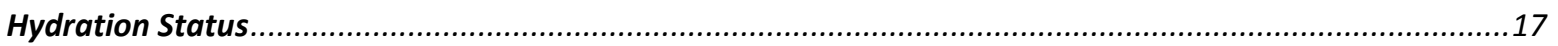

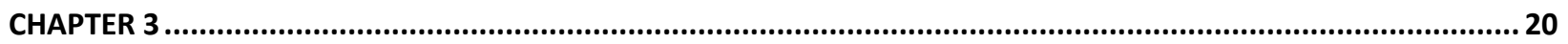

METHODS …

Subjects

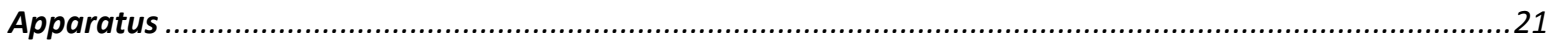

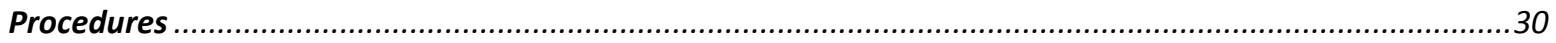

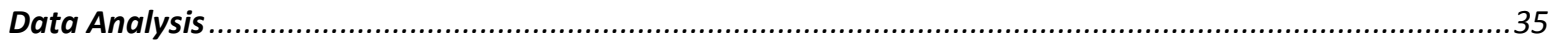

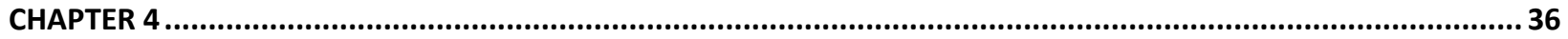

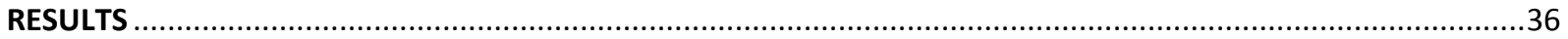

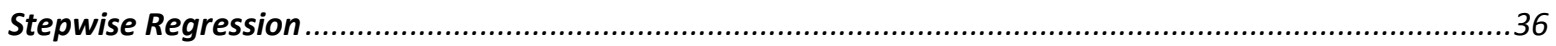

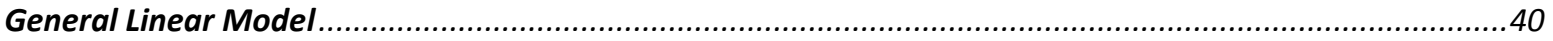

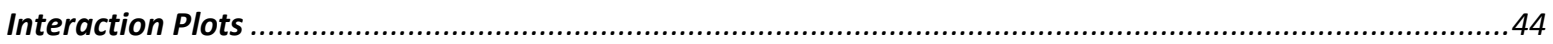

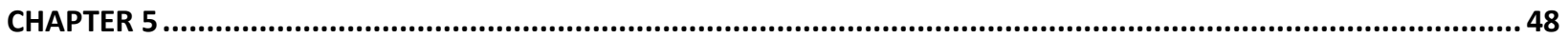

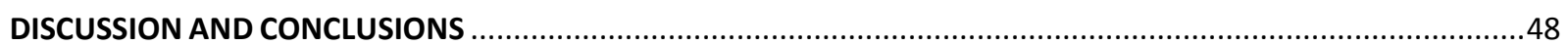

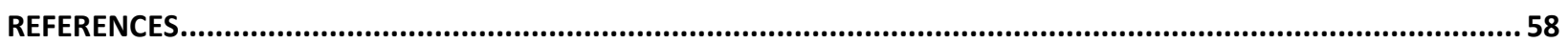

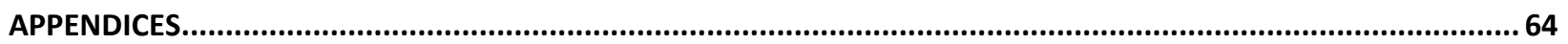

APPENDIX A. INSTITUTIONAL REVIEW BOARD HUMAN RESEARCH SUBJECTS PROTOCOL STATEMENT ....................................64

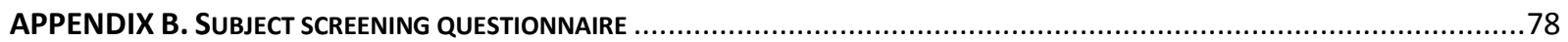

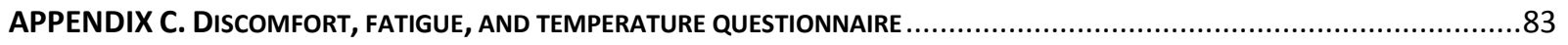

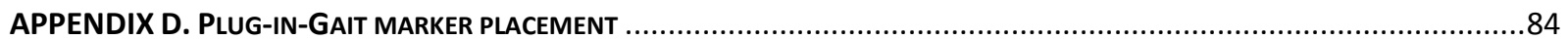

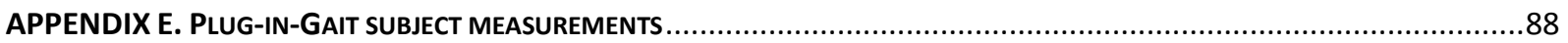

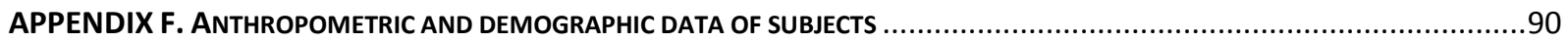

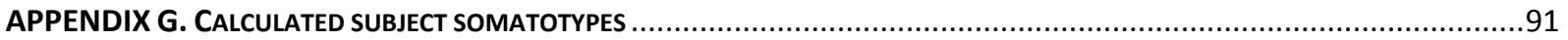




\section{LIST OF TABLES}

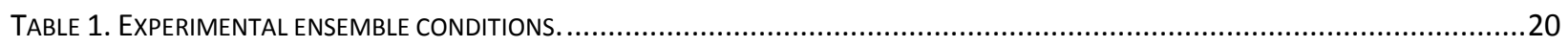

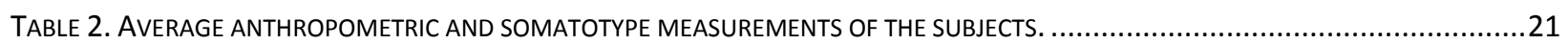

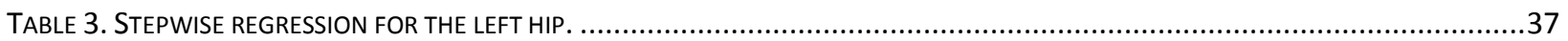

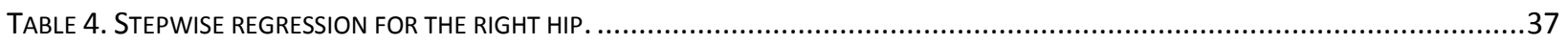

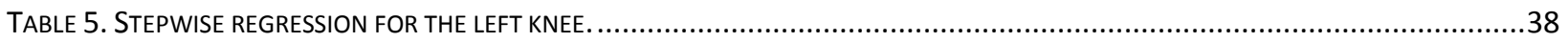

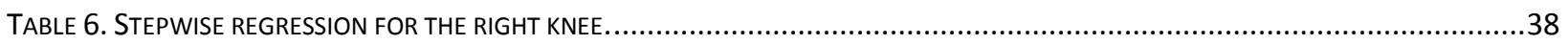

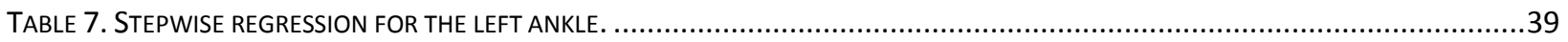

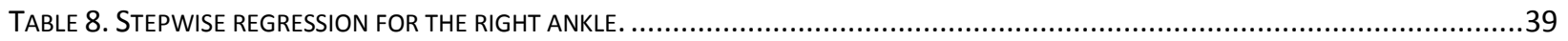

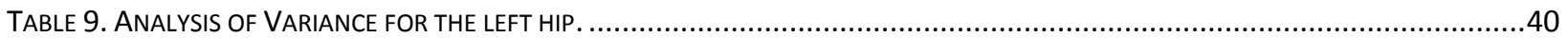

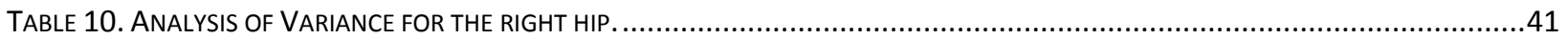

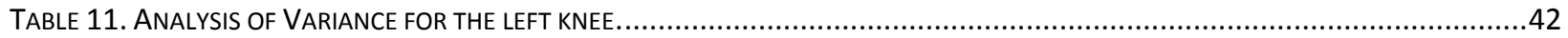

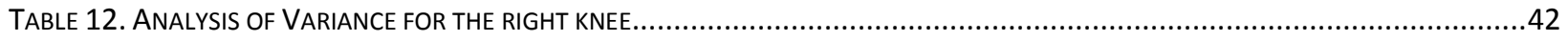

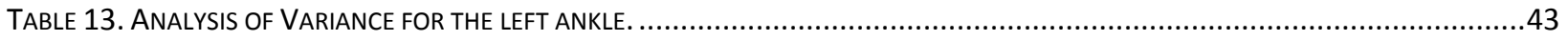

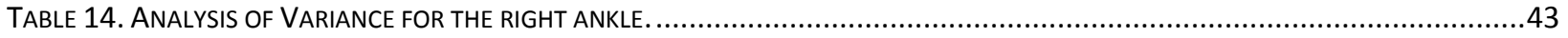




\section{LIST OF FIGURES}

FIGURE 1. PLUG-IN-GAIT SPATIAL MOdEL (LIU, LIAN, \& LIU, 2008) FOR MARKER PLACEMENT..................................................22

FIGURE 2. VICON MARKER AND JVC VIDEO RECORDING CAMERAS, KISTLER FORCE PLATES, AND BOX PLACEMENTS..........................23

FIGURE 3. MACHINED “BOX” USED AT FLOOR LIFTING STATION, WITHOUT CALIBRATED WEIGHTS...............................................24

Figure 4. Placement of dermal patches on body at locations of 1) Left SCAPUla, 2) Left thigh, 3) Left SHANK, AND 4) RiGHT FOREARM, MODIFIED FROM MODEL BY MITCHELL AND WYNDHAM (1969) ............................................................25

FIGURE 5. DISCOMFORT, FATIGUE, AND TEMPERATURE SURVEY ADMINISTERED AT QUESTIONNAIRE STATION, MODIFIED FROM WIKER,

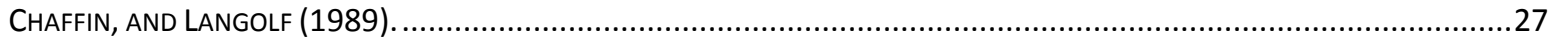

FIGURE 6. VELCRO ${ }^{\circledR}$ BAND (WITH SPRINGS) AND FOAM BARRIER FROM TOP TO BOTTOM, RESPECTIVELY ........................................28

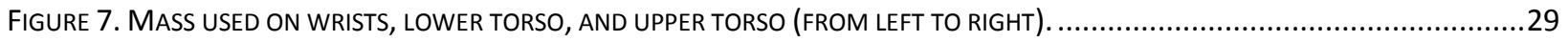

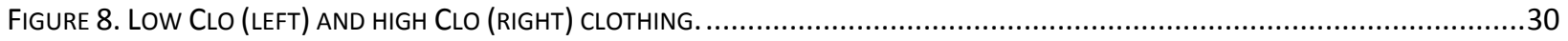

FiguRE 9. EXAMPLE OF CLOTHING ENSEMBLES, FROM LEFT TO RIGHT, WITH SUBJECT IN LOW CLO AND HIGH CLO WITH MASS AND BANDS.

.32

FIGURE 10. CYCLIC WORK PROTOCOL FOLLOWED FOR STUDY; THE ORDER OF ARM LIFT AND TREADMILL STATIONS WAS INTERCHANGED FOR

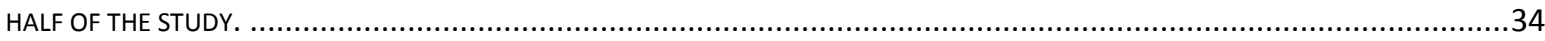

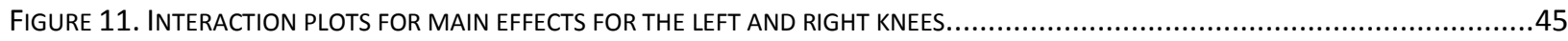

FIGURE 12. INTERACTION PLOTS FOR TWO-WAY INTERACTIONS FOR THE LEFT AND RIGHT KNEES............................................46

FIGURE 13. INTERACTION PLOTS FOR THREE-WAY INTERACTIONS FOR THE LEFT AND RIGHT KNEES..........................................47

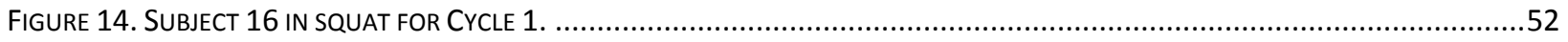

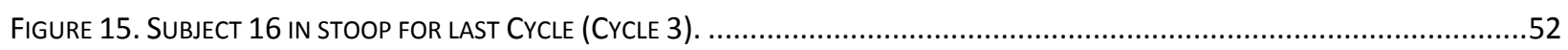

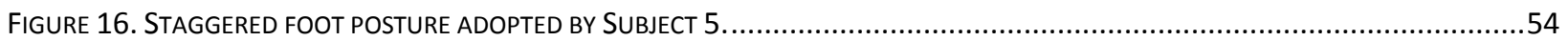




\section{CHAPTER 1}

\section{INTRODUCTION}

In the field of ergonomics, the capabilities and limitations of humans are studied, and the observations are applied towards improving jobs and tasks. Ergonomic evaluations are also used to ensure that the demands of a job do not exceed the capabilities of a worker (A. Garg, Chaffin, \& Herrin, 1978). Of particular concern is that of developing a better job design, which reduces the risk of injuries and illnesses in the workplace, and improves overall performance of the job. However, it is difficult to measure the stresses of the workers performing jobs in the field. To accomplish this, there is interest in developing a sensor suit that can be placed on workers. The suit would be able to record the information needed to assess the job, and it would be necessary for it to not interfere with worker performance. In this study, outfit ensembles were analyzed to determine whether or not they have an effect on posture while performing lifting tasks. The ensembles used in this study included combinations of Clo, Mass, and Banding, with high and low conditions for each (a $2^{3}$ design), and the goal was to analyze the data and determine if the Clo, Mass, and Banding, whether alone or with interactions, intruded upon posture. These ensembles were chosen so as to mimic the sensor suit components that would be placed on an individual in the field for analyzing posture, where Clo, Mass and Banding could be clothing, battery packs, and tape bandages to secure sensors, respectively. The postures that were analyzed were those of stoop/squat lifts; lifts where subjects were asked to lift a box from the floor to waist height, hold, and then return to the floor. 
In this study, it was hypothesized that the ensemble conditions with added Banding, Clo, and Mass would adversely affect the lifting strategies of the subjects. Under these conditions, subjects were expected to transition to a more stooped posture, due to its lesser physiological demand. 


\section{CHAPTER 2}

\section{LITERATURE REVIEW}

The scope of this literature review was to include research that had previously been done on the various aspects of this study. While the postures used during the performance of a lifting task were of primary concern, it was also necessary to review research that pertained to the ensembles, equipment, and protocol being used.

\section{Lifting Tasks}

Lifting tasks are one the many types of activities that comprise the group of manual materials handling $(\mathrm{MMH})$ jobs. Manual materials handling jobs are prevalent in the workplace, and demand that a certain focus be put on them, so as to minimize the risk of injuries and illnesses with which they are associated. The hazards that are associated with lifting typically tend to be characterized by either biomechanical, which focuses on loads of force on the musculoskeletal system, or metabolic criteria, which deals with endurance capacities of the body (A. Garg \& Herrin, 1979). When performing a lifting task, the two postures generally of consideration are that of a stoop (being at the waist) or squat (bending at the knees). Research by Garg and Herrin (1979) found that, in terms of the biomechanics, when a load was placed closer to the body, only small differences in the compressive forces between a stoop and squat existed at the $L_{5} / S_{1}$ disc of the back; however, the squat was able to minimize the tension of the erector spinae muscle. With the load placed further away from the body, the stoop was found to minimize compressive forces at $L_{5} / S_{1}$. As far as the metabolic analysis for the two lifting postures were concerned, the stoop was found to result in lower metabolic expenditure rates, 
as well as lower heart rates. Also, in comparing the squat to the stoop, the squat would require more energy than the stoop posture, therefore making a larger contribution to physical fatigue.

Many musculoskeletal disorders have been attributed to $\mathrm{MMH}$, especially those involving lifting and lifts with repetitions (Sanchez \& Grieve, 1992). In situations requiring repetitive handling, both cardiovascular and muscular fatigue may develop, thus being the limiting factor as far as worker acceptability of the task is concerned. Additionally, in the overall context of manual handling, there remains debate over the level of spinal compressive force which may be regarded as being safe (Nicholson, 1989). Epidemiological studies have frequently reported on lifting and its association with low back pain (Lavender, Li, Andersson, \& Natarajan, 1999). Back pain is the most expensive industrial injury, and it has been estimated that approximately $80 \%$ of the general population will have a complaint of low back pain and disability in the course of their lives. Not only can improper body mechanics cause or exacerbate most episodes of acute low back pain, but twisting can change the dynamics of spinal loading and place undue stress on soft tissues and weight-bearing surfaces (Neal, 1997).

Symmetric activities are less stressful than asymmetric when performing a full stoop (Gallagher, 1991). It has been reported that asymmetric lifting tasks are physically more difficult than symmetric lifting (Hattori et al., 1996). Trunk asymmetry has been found to create hazardous conditions for lower back structures and reduces lifting capability; it is also harmful to back structures, and affects lifting performance. Even during sagittal-symmetric lifting, lateral bending and twisting moments act on the spine, and these moments increase with either heavier weights or with faster lifts (Lavender, et al., 1999). Psychophysical tests have determined that the maximal acceptable load a worker can lift is significantly lower from 
asymmetric positions than from symmetrical positions (Gagnon, Plamondon, Gravel, \& Lortie, 1996). Ratings of perceived exertion (RPE) in the lower back and the lower body in asymmetric lifting have suggested higher stress than those in symmetric lifting; for many body sites in asymmetric lifting, the RPEs indicated that they were more stressful than those in symmetric lifting (Hattori, et al., 1996).

According to Garg and Saxena (1979), there is no clear evidence to suggest that there is any advantage to be gained from the use of the squat method of lifting from physiological, biomechanical, or epidemiological considerations. Based upon a biomechanical analysis, the squat method could not be recommended on the basis that it would create lower compressive forces at the L5/S1 disc (Arun Garg \& Saxena, 1979). Other researchers have concluded that lifting with the lumbar spine in lordosis is the safest and most effective way to perform a lift, as this posture can reduce the intervertebral disc pressure (Schenk, Doran, \& Stachura, 1996). In a study by Hattori et al. (1996), stoop lifting was presumed to place a greater load on the lower back than squat lifting. Additionally, the postural factor has a significant effect on height at peak force, which is higher in squat lifting than in stoop lifting; during squat lifting, subjects were observed to have first begun to straighten their knees and then lift the box using their legs and upper bodies (Hattori, et al., 1996). When the moment at the L5/S1disc increased, the corresponding knee joint moment decreased; this indicated that as the external weight load increased, the lifting technique changed more to a back lift than a leg lift (Gagnon, et al., 1996). Leg lifting with a squat position is metabolically more demanding, possibly leading to more fatigue related injuries (Arun Garg \& Saxena, 1979). The metabolic cost is lower with a straightknee (stoop) than with a bent-knee (squat) method, indicating that these changes in efforts and 
positions also affect task efficiency (Gagnon, et al., 1996). While the metabolic cost is the greatest for a squat, it is the smallest for a freestyle lift (an individual's preferred lifting style); therefore, based on subjective estimates of physical fatigue, work can be performed if the workers are allowed to use the freestyle lifting technique (Arun Garg \& Saxena, 1979).

Safe loads for lifting are difficult to establish, and strength is affected by body posture (Sanchez \& Grieve, 1992). Work by Garg and Saxena (1979) have shown that the maximum workloads that are acceptable to the workers were significantly affected by both lifting frequency and technique. Lifting weights at higher frequencies has been shown to be more demanding metabolically than lifting heavier weights at lower frequencies, and the frequency has also been related to an increased incidence of low back pain. Also, the higher the frequency of lifting heavy objects on a job, the greater the frequency and severity of back, musculoskeletal, and contact injuries. Maximum acceptable workloads were found to be the largest for the freestyle lifting method, and the maximum acceptable workload is not only significantly affected by lifting frequency, but also technique. Therefore, an optimum physiological lifting frequency was determined to be around 9 lifts/min for lifting from floor to $0.5 \mathrm{~m}$ height (Arun Garg \& Saxena, 1979).

\section{Posture, Movement, and Mass}

In the workplace, MMH tasks involve the bending of the torso (M.J. Jorgensen, Marras, \& Gupta, 2003). With the lifting and lowering in these tasks, as the torso flexes forward in the sagittal plane, the extensor muscle group of the spine at the lower lumbar levels becomes closer to the spine (Michael J. Jorgensen, Marras, Gupta, \& Waters, 2003). It has been suggested that torso flexion alters the muscle geometry of the lumbar back muscles (M.J. 
Jorgensen, et al., 2003), and that as the torso flexes forward, the sagittal plane moment arms of the lumbar erector mass decrease (Michael J. Jorgensen, et al., 2003). In order to maintain control of balance, control of the upright trunk is crucial (McGibbon \& Krebs, 2001).

Research has suggested that underlying components of the organization of postural strategies could include global postural goals such as an upright trunk, center of mass equilibrium, head stability, and their weighted combinations (Buchanan \& Horak, 2001). Body posture has been found to influence blood pressure and heart rate, with an upright posture having a higher heart rate and diastolic pressure compared to supine and seated positions (Steptoe, 2000). When considering the effect of exercise on posture, exercise can impair postural stability by interfering with the efficacy of visual input to postural control. This impairment of stability has been found to exist when on a treadmill; after running on a treadmill, balance may become impaired as a result of the conflict of information between somatosensory and visual input. Even exercise of a moderate intensity can deteriorate the visual contribution to postural stability, and running tends to disturb postural stability more so than walking, possibly due to more excessive head movement and disturbance of vestibular and visual information centers (Derave, Tombeux, Cottyn, Pannier, \& De Clercq, 2002).

The stance of an individual can greatly affect the posture of an individual, as research has shown significant differences to exist between wide and narrow standing stances. Perturbations in narrow stance have been shown to result in larger lateral flexion of the trunk that is accompanied by trunk rotation and flexion, which may be related to coupling of rotation in active, purposeful trunk motion. Additionally, no significant changes in body center of mass displacement due to large changes in center of pressure have been found, despite more trunk 
displacement that occurs in narrow stance. On the other hand, in wide stance, there is less trunk motion to control and a relaxation of the horizontal force constraint, due to increased stability and passive stiffness of the musculoskeletal system. Although there are differences that exist between extreme stances, the center of mass of the body is effectively regulated in that the center of mass is not displaced to any greater degree in narrow versus wide stance (Henry, Fung, \& Horak, 2001).

Posture and movements can be affected by additional body mass, as is experienced with obese individuals and pregnant women (Gilleard \& Smith, 2007; Jensen, Doucet, \& Treitz, 1996; Paul, Sallé, \& Frings-Dresen, 1996). Obese subjects have been found to have difficulties with balance, possibly making these individuals more cautious in moving forward to the end of range for a movement. In the obese, trunk forward flexion motion has shown to be restricted in both sitting and standing. Furthermore, a higher body mass index (BMI) has been correlated to an increase in angular displacement of the thoracic segment and decrease in the thoracolumbar spine range of motion, which indicated that an increase in adiposity can lead to even further motion restriction (Gilleard \& Smith, 2007).

However, excessive anterior trunk tissue was not shown to alter pelvis segment forward flexion motion. A strategy that may have been used to minimize obstruction of the pelvis during forward flexion tasks was that of a wider stance (Gilleard \& Smith, 2007). In pregnant women, changes in segment mass and mass distribution may impact loads on various joints (Jensen, et al., 1996; Paul, et al., 1996). During the course of the second and third trimesters, lower trunk moments of inertia increased at a greater rate than all other segments, while no significant differences were found between the remaining segments (Jensen, et al., 1996). Additionally, 
the hip joint load will increase due to the increase in segment mass, forward shift of the trunk center of mass, and the postural changes due to pregnancy (Paul, et al., 1996).

\section{Energy Expenditure}

Energy is the capacity for performing work (Jackson \& Kinney, 1978), and energy expenditure has been defined as being the rate that heat is produced by the body (SartonMiller, 2006). Energy expenditure is of importance since the process of adapting a work task to be consistent with the capacity of the worker is dependent upon the ability to determine the caloric cost of the work (Balogun, 1988). Furthermore, an accepted standard reference for physical activity is that of the energy expenditure due to physical activity (Bouten, Koekkoek, Verduin, Kodde, \& Janssen, 1997). Research by Abitbol (1988) found that respiratory and heart rates, as well as temperature, could be used as indicators of energy expenditure; temperature was also found to be an indicator of oxygen consumption. Other research found that energy expenditure, heart rate, and electrical activity of muscles during work could be used as indicators in establishing the physical workload involved for a given job or task (Vezina, Tierney, \& Messing, 1992). Once physical work is performed, energy demand increases. In performing an evaluation of a physical workload, the changes in heat production and energy liberation for muscle activity are important to consider. The heart rate of an average adult is 72 beats per minute under normal resting conditions, and a person can be safely engaged in work strenuous enough to increase the heart rate to 170 beats per minute; however, it has been recommended that the combined stress of the task and environment should not create enough strain that the heart rate exceeds 110 beats per minute (Salokhe \& Mamansari, 1995). 
Metabolic expenditure rates are important to consider when looking at the amount of work that can be done by an individual, particularly when it is desired for a task to be performed at maximum intensity, but without the development of excessive fatigue (A. Garg, et al., 1978). For a young, healthy male, the rate that was found to be acceptable for physical work done continuously during an eight-hour work day was that of $5.2 \mathrm{Kcal} / \mathrm{min}$ (A. Garg, et al., 1978; A. Garg \& Herrin, 1979). However, while $5.2 \mathrm{Kcal}$ was found to be the acceptable metabolic rate, Garg et al. (1978) were interested in predicting the energy requirements for various tasks. Assuming that $\mathrm{MMH}$ tasks could be broken into simpler tasks, models were able to be created for the overall, more complex task.

Energy expenditure is an important consideration when interested in postures, and the position and time within a given posture exert a significant effect on energy expenditure (Jackson \& Kinney, 1978). Standing is more expensive energetically than sitting or lying in a supine posture (Roefs, Schrama, \& Schouten, 1996), and when the body is upright with the weight of the body evenly distributed between both feet, minimal energy expenditure is required to maintain the balance (Savage, Toth, \& Ades, 2007). If the trunk of the body is flexed, the rate of rate of energy expenditure will be higher with the increasing trunk flexion, due to the additional muscle activity required to hold the trunk and head against gravity and maintain a standing balance (Saha, Gard, Fatone, \& Ondra, 2007). The addition of a load, such as in a MMH task, will also have an impact upon energy expenditure, as a load center of mass that is kept as close to the body center of mass as possible will result in a lower energy cost, and tends to keep the body in an upright posture, similar to that of unloaded walking (Knapik, Reynolds, \& Harman, 2004). When walking, the energy expenditure is twice as much as when in 
a supine posture (Abitbol, 1988); however, if walking on a treadmill, the energy expenditure may be altered if the side bars of the treadmill are used (Jaegers, Vos, Rispens, \& Hof, 1993).

Body weight is another consideration in the rate of energy expenditure. Research on obesity has found body weight to be the most important factor for the increased oxygen cost of muscular work, which is due to an increased cost of keeping the body stable in the upright position, as well as the cost of moving the heavy limbs (Freyschuss \& Melcher, 1978). This is also able to be applied to pregnant women, as the gain in body weight during pregnancy contributes to an increase in energy cost in performing work; also during pregnancy, basal energy expenditure has been shown to increase by 13 to 37 percent. In pregnant women, although heart rate and energy expenditure change with pregnancy, the relationship between heart rate and energy expenditure do not (Blackburn \& Calloway, 1985).

\section{Banding}

Bandaging has been used for various applications, including clinical use (Botti, Williamson, Steen, McTaggart, \& Reid, 1998), the treatment of wounds (Bale \& Harding, 1985), fractures (Eagen, SooHoo, \& Cracchiolo, 2006), and deep vein thrombosis (Brady et al., 2007). Compression also has been applied to parts of the body for other applications, including the use of compression stockings and hosiery for daily use (Kraemer et al., 2000; Watanuki \& Murata, 1994), or even tourniquets for medical procedures (Hagenouw, Bridenbaugh, van Egmond, \&

Stuebing, 1986). A concern when applying any of these treatments is that the individual will experience discomfort.

Bale and Harding (1985) reported that in the treatment of wounds, when a silastic foam dressing was used on patients, it was reported that comfort was felt with this type of dressing, 
as opposed to that of a paraffin gauze dressing, which can adhere to the wound, and thereby making removal uncomfortable. Evidence from clinical use has shown that pressure bandaging caused much discomfort in the patients to whom it has been applied. In patients having undergone coronary angiography, the application of pressure bandaging (applied over the femoral puncture site, and wrapped around the leg, lower abdomen, and lower back) resulted in a higher incidence of back and leg pain. Patients with the pressure bandages experienced more discomfort and required more medication than those without; it was thought that pressure bandages may also result in a generalized perception of discomfort, thereby reducing the patients' tolerance to pain or discomfort (Botti, et al., 1998).

Compression dressing has also been applied under a fiberglass cast, so as to accommodate for swelling and tissue expansion associated with a fracture. Typical casts have not been found to be suitable immediately after surgery since they are unable to accommodate significant swelling. A Jones dressing, which is a bulky, well-padded compression bandage (Kawabata, Obara, Komiyama, \& Narumi, 2001), is used by some surgeons to avoid the postoperative problems caused by swelling and increased pressure beneath casts. While some studies have suggested that the only cast that is safe to use in the immediate postoperative period for ankle fractures is a cast that has been split and spread, the approach using compression dressing under a fiberglass cast has seemed to accommodate the swelling in all patients, and there were found to be no complaints of excessive pain (Eagen, et al., 2006).

Compressive stockings have been worn to reduce the significant amounts of fatigue and body discomfort experienced at the end of the workday, associated with long periods of standing (Kraemer, et al., 2000), as well as to reduce deep vein thrombosis (Brady, et al., 2007). 
In wearing the compressive stockings or hosiery to lessen workday fatigue and discomfort, significant reductions were found in the size of the ankle and calf, but none in the thigh circumference (Kraemer, et al., 2000). However, another study reported that the subjects complained of discomfort due to the high pressure that was applied to the skin by wearing the stockings (Watanuki \& Murata, 1994). For the use of the stockings for deep vein thrombosis, some research has suggested that the condition is not reduced or improved by compression of the thigh or calf. Additionally, It has been reported that patients with deep vein thrombosis will refuse to wear the compressive stockings if they are found to be uncomfortable (Brady, et al., 2007).

Hagenouw et al. (1986) have found that the use of tourniquets results in intolerable pain at the site of application, as only half of the experiments were able to be concluded. Tourniquet-induced pain during spinal or epidural anesthesia has been noted to occur $60-90$ minutes after inflation of the tourniquet. With half the experiments being terminated due to intolerable pain at the site of the tourniquet, it is possible that the true incidence of tourniquetinduced pain during otherwise satisfactory regional anesthesia is higher than typically thought (Hagenouw, et al., 1986).

\section{Temperature}

Core body temperature is one of the most tightly regulated human physiological parameters (Lenhardt, 2003), and can influence exercise performance, making it essential to monitor when studying the temperature regulation of the human body during exercise (Byrne \& Lim, 2007). While it has been reported that exercise intensity and the inability to dissipate metabolic heat are the most influential factors affecting the rise in core temperature (Laursen 
et al., 2006), it has also been reasoned that the rise in core temperature during exercise was not due to an inability to dissipate the increased metabolic heat load, but rather a change in regulatory adjustments of body temperature (Gisolfi, 1983). While a more rapid rise in core temperature is consistent with the concept that increase in body temperature is the critical factor that limits exercise in hot ambient conditions (Rowland, Garrison, \& Pober, 2007), core temperature has also been found to rise with increasing intensities of steady-state exercise, independent of ambient temperature (Gisolfi, 1983).

Since it is desirable to monitor core temperature, many locations have been proposed on which to perform the measurements. If a core temperature site is unable to be used for a particular clinical setting, appropriate sites may include that of the bladder, rectum, axilla, and skin. Additionally, the tympanic membrane has become a preferred site in thermoregulatory research. Of the various locations that can be used for core temperature measurements, rectal temperature monitoring has been found to be the most accurate and precise under steadystate conditions (Cattaneo et al., 2000; Howe \& Boden, 2007; Lenhardt, 2003). However, rectal temperature should be used cautiously, as it typically lags behind that measured in core sites during extreme thermal perturbations, becoming less accurate as rapid changes in core temperature occur (Lenhardt, 2003; Woodrow et al., 2006).

Although literature lacks consensus about the best site for the measurement of core temperature (Woodrow, et al., 2006), the pulmonary artery and the esophagus have been found to be preferred sites for monitoring core temperature. Although the pulmonary artery temperature is usually not practical to measure, it has been considered to be ideal for core temperature (Woodrow, et al., 2006), as it is the most representative location since the 
observed temperature is a result of the convective mixing of blood from the entire body (Byrne \& Lim, 2007; Shellock \& Rubin, 1982). Other research has suggested that the esophagus provides for the most valid measurement of core temperature (Sookram et al., 2004), and that it is the preferred site for this measurement, since it provides an indirect measure of the temperature of the arterial blood leaving the heart (Edwards, Belyavin, \& Harrison, 1978).

A convenient method for monitoring core temperature is that of an ingestible telemetric temperature sensor (pill), which not only represents a valid index of core temperature, but also provides the additional benefit of the subject being unaware of its presence once it has been swallowed (Byrne \& Lim, 2007). The use of a telemetric temperature sensor is also of value when body temperature must be closely monitored (Newsom, Bolgos, Colby, \& Nemzek, 2004). However, while the intestinal temperature being monitored by telemetry is more receptive to changes than that of rectal temperature, one possible issue with an ingestible sensor is its ability to migrate. Since it may be able to move along the gastrointestinal tract, the possibility of temperature gradients could have an effect on the temperature measurement (Byrne \& Lim, 2007).

Skin temperature can be monitored by performing measurements at various sites. Ramanathan (1964) developed a simple weighting formula using only four sites (chest, arm, and leg) to determine mean surface temperature; previous systems considered in designing this formula were that of the Hardy-Dubois system and Burton's simple formula. Not only were whole limbs taken into consideration with the four-point system, but also the weighting for each segment allowed for a simple computation (Ramanathan, 1964). Research by Mitchell and Wyndham (1969) confirmed that Ramanathan's simple weighting formula with only four points 
of measurement and single-digit coefficients provided a fairly good rating, and recommended it where simplicity and a large amount of measurements are required. However, an optimal method for the calculation of the weighted mean skin surface temperature was Winslow's 15point system (D. Mitchell \& C. H. Wyndham, 1969).

\section{Heat and Related IIInesses}

Hot and humid environments can significantly add to the challenge that physical exercise imposes on the human thermoregulatory system, since heat exchange between the body and environment is substantially impaired under these conditions (Wendt, van Loon, \& van Marken Lichtenbelt, 2007). A high ambient temperature can dramatically reduce endurance exercise performance (Rowland, et al., 2007), and heat exposure limits should take into consideration the cumulative effects of heat stress and ergonomics stressors on cardiac response, so as to prevent cumulative fatigue and possible long-term health effects (Brabant, Bédard, \& Mergler, 1989).

Excessive heat exposure can lead to profuse sweating, which is then paired with inadequate fluid and electrolyte intake. After this, muscle spasm or muscle cramps can result, which are one of the earliest signs of heat illness. Typically, exertional heat illness is the result of increased heat production and impaired heat dissipation (Howe \& Boden, 2007); also, dehydration is a major modifiable risk factor for and key precursor to heat illness (Coris, Ramirez, \& Van Durme, 2004; Howe \& Boden, 2007). Dehydration is able to be determined by both inadequate fluid intake and excessive fluid loss primarily by means of sweating (Howe \& Boden, 2007). During exercise, if the core temperature rises due to hot and humid weather conditions or dehydration, there is an increased risk of developing heat illness (Wendt, et al., 
2007). Being able to immediately identify and provide effective treatment to the milder forms of heat illness are crucial to the prevention of heat stroke and potentially related fatalities (Coris, et al., 2004; Howe \& Boden, 2007). Malaise, fatigue, and dizziness are often signs of heat exhaustion, and if not treated, it has the potential to progress to heat stroke (Howe \& Boden, 2007).

While exertional heat stroke is entirely preventable (Howe \& Boden, 2007), if it should occur, it needs to be understood that heat stroke is a medical emergency and that if effective treatment is delayed or withheld through non-recognition, mortality rates of up to $80 \%$ may be recorded (Kielblock, Van Rensburg, \& Franz, 1986). An inconsequential diagnostic criterion for heat stroke is the presence or absence of sweating, and a major determinant for the outcome of heat stroke is the duration of hyperthermia (Howe \& Boden, 2007). Heat stroke represents a condition in which elevated body temperatures are casually related to tissue damage of an irreversible nature (Kielblock, et al., 1986), and treatment consists of reducing the heat as quickly as possible, and monitoring for any complications related to heat exposure (Howe \& Boden, 2007).

\section{Hydration Status}

It has been suggested that the maintenance of a normal state of hydration is thought to be critical for the prevention of heat-related illness, since dehydration is believed to play a role in hyperthermia (Godek, Godek, \& Bartolozzi, 2005). The level of dehydration has been found to be involved with a higher rectal temperature, thereby increasing risk of heat illness (Morante \& Brotherhood, 2007). An additional rise in core temperature during dehydration may be explained by inadequate internal heat transfer and the reduced heat dissipation because of 
reduced evaporation during transient phase of sweating (Candas, Libert, Brandenberger, Sagot, \& Kahn, 1988). Dehydration has been shown to have a negative impact on not only exercise, but also physiological function, and when exercising in the heat, consuming fluids can be quite valuable (Byrne, Lim, Chew, \& Ming, 2005). Adequate hydration can help by reducing the risk of profound heat stress and dehydration that can occur in individuals working in hot environments (Meyers, Horrigan, \& Lotz, 1995). Temperature regulation and work performance in the heat are critically dependent on the state of body hydration (Meyers, et al., 1995), and the state of body hydration has a profound influence upon both the ability to regulate internal body temperature, and maintain circulatory stability during prolonged heat exposure or extended periods of continuous exercise (Nadel, Fortney, \& Wenger, 1980). In order to avoid dehydration, sweat loss must be matched by fluid consumption once sweating becomes the primary means of heat dissipation (Wendt, et al., 2007). If the body fluid losses due to profuse sweating during prolonged exercise in the heat are not compensated, large increases in the heart rate and core temperature will be induced (Candas, et al., 1988). The main goal of fluid replacement during prolonged exercise under heat stress is the prevention of heat illness due to the detrimental effects of dehydration on body temperature regulation and cardiovascular function; during military physical activity in the heat, Army recommendations for the replacement of fluids advocate the ingestion of plain water (Byrne, et al., 2005). Howe and Boden (2007) recommend the consumption of 16 ounces of water or sports drink 1 hour before exertion, and continued hydration with 4 to 8 ounces of fluid every 15 to 20 minutes for the duration of the exertion to manage fluids. 
Hypohydration is a critical consideration for individuals performing physical activity, as this reduction in total body water can increase physiologic strain, decrease physical as well as cognitive performance, attenuate resistance exercise performance, reduce cardiac output during exercise, and increase the susceptibility to heat injury or illness (Judelson et al., 2007; Meyers, et al., 1995; Montain, Sawka, Latzka, \& Valeri, 1998; Nadel, et al., 1980). When in a state of hypohydration, the performance in prolonged exercise may be affected due to a decreased sweat rate and an increased core temperature (Rico-Sanz et al., 1996). In one study, hypohydration was found to significantly attenuate performance of an isotonic, multirepetition, multi-set exercise routine that was typical of conventional resistance exercise, while it was found to have little demonstrable effect on single, maximal-effort strength and power (Judelson, et al., 2007). Other research has found heat acclimation state and aerobic fitness to be factors having an influence upon the magnitude of thermal penalty associated with hypohydration during exercise heat stress (Montain, et al., 1998). Hyperhydration, on the other hand, while it has been shown to cause reductions in heart rate, no changes have been found in internal body temperature or in the cutaneous blood flow response to body heating during exercise (Nadel, et al., 1980). Research by Rico-Sanz et al. (1996) found that, in soccer players, there was a reduction in thermal stress during the match as a function of environmental heat stress index in the hyperhydration condition, and that there was no significant effect of hyperhydration on fatigability during the performance of one intense set of knee flexion and extension. 


\section{CHAPTER 3}

\section{METHODS}

A $2^{3}$ factorial, between-subjects design was used, producing 8 experimental ensemble conditions for levels of Clo, Mass, and Banding, as shown in Table 1 (where + and - represent the high and low conditions, respectively), with an individual subject exposed only to one ensemble combination.

Table 1. Experimental ensemble conditions.

\begin{tabular}{lccc}
\hline & CLO & Mass & Banding \\
\hline \hline Ensemble 1 & - & - & - \\
Ensemble 2 & - & - & + \\
Ensemble 3 & - & + & - \\
Ensemble 4 & - & + & + \\
Ensemble 5 & + & - & - \\
Ensemble 6 & + & - & + \\
Ensemble 7 & + & + & - \\
Ensemble 8 & + & + & + \\
\hline
\end{tabular}

Kinematic recordings of the hip, knee, and ankle for complete ground-lift and waist-toground exertion sequences recorded during the five-minute exertion epochs over the course of the two-hour period served as the dependent variables for this study. Independent variables were Subject, Banding, Clo, Mass, Cycle, Part, and Phase. Cycle was defined as being one of the six twenty-minute cycles comprising the two-hour period, and is discussed in detail later. Part was one of three lifts that was extracted from the beginning $\left(1^{\text {st }}\right.$ lift), middle $\left(15^{\text {th }}\right.$ lift), and end $\left(28^{\text {th }}\right.$ lift) of each 5 -mintute recording. Phase was one of the four motion components for each lift (lowering to get the box, lifting the box, lowering the box to the floor, and ascending without the box). Anatomical joint angles for the ankle, knee, and hip were determined from each Part (beginning, middle, and end) to examine changes with exposure duration. This 
sampling provided an opportunity to detect duration-based changes while reducing kinematic analysis burden to $18 \mathrm{lift} /$ lower sequences during a full two-hour experiment. All hypotheses were tested using Type I errors of 0.05 or less.

\section{Subjects}

Thirty-two subjects (22 men, 10 women; average age of 27.53 years, SD 7.27), who reported that they were in good health with no history of low back pain, participated in this study on a paid and informed consent basis. All subjects received $\$ 10$ per hour for their participation in the two-hour study. See Table 2 for subject anthropometric information.

Table 2. Average anthropometric and somatotype measurements of the subjects.

\begin{tabular}{rcc}
\hline & Average & $\begin{array}{c}\text { Standard } \\
\text { Deviation }\end{array}$ \\
\hline \hline Height $(\mathrm{cm})$ & 173.34 & 32.63 \\
Stretch Stature $(\mathrm{cm})$ & 173.37 & 32.68 \\
Body Mass $(\mathbf{k g})$ & 74.51 & 18.81 \\
Triceps Skinfold $(\mathrm{mm})$ & 16.31 & 7.71 \\
Subscapular Skinfold $(\mathrm{mm})$ & 16.52 & 6.51 \\
Supraspinale Skinfold $(\mathrm{mm})$ & 13.44 & 7.19 \\
Medial Calf Skinfold $(\mathrm{mm})$ & 11.38 & 6.02 \\
Humerus Breadth $(\mathrm{cm})$ & 6.70 & 1.37 \\
Femur Breadth $(\mathrm{cm})$ & 11.42 & 8.18 \\
Upper Arm Girth $(\mathrm{cm})$ & 30.73 & 6.47 \\
Calf Girth $(\mathrm{cm})$ & 36.66 & 7.03 \\
\hline
\end{tabular}

\section{Apparatus}

Kinematic and Kinetic Recording Apparatus. Eight VICON MX-13 near-infrared cameras recorded the locations of 39 retro-reflective markers that were positioned on subjects using the Plug-in-Gait spatial model shown in Figure 1, and described elsewhere (Bell, Pedersen, \& Brand, 
1990; Davis, Ounpuu, Tyburski, \& Gage, 1991; Ramakrishnan, Kadaba, \& Wootten, 1987; Ramakrishnan, Masiello, \& Kadaba, 1991; Ramakrishnan, Wootten, \& Kadaba, 1989).

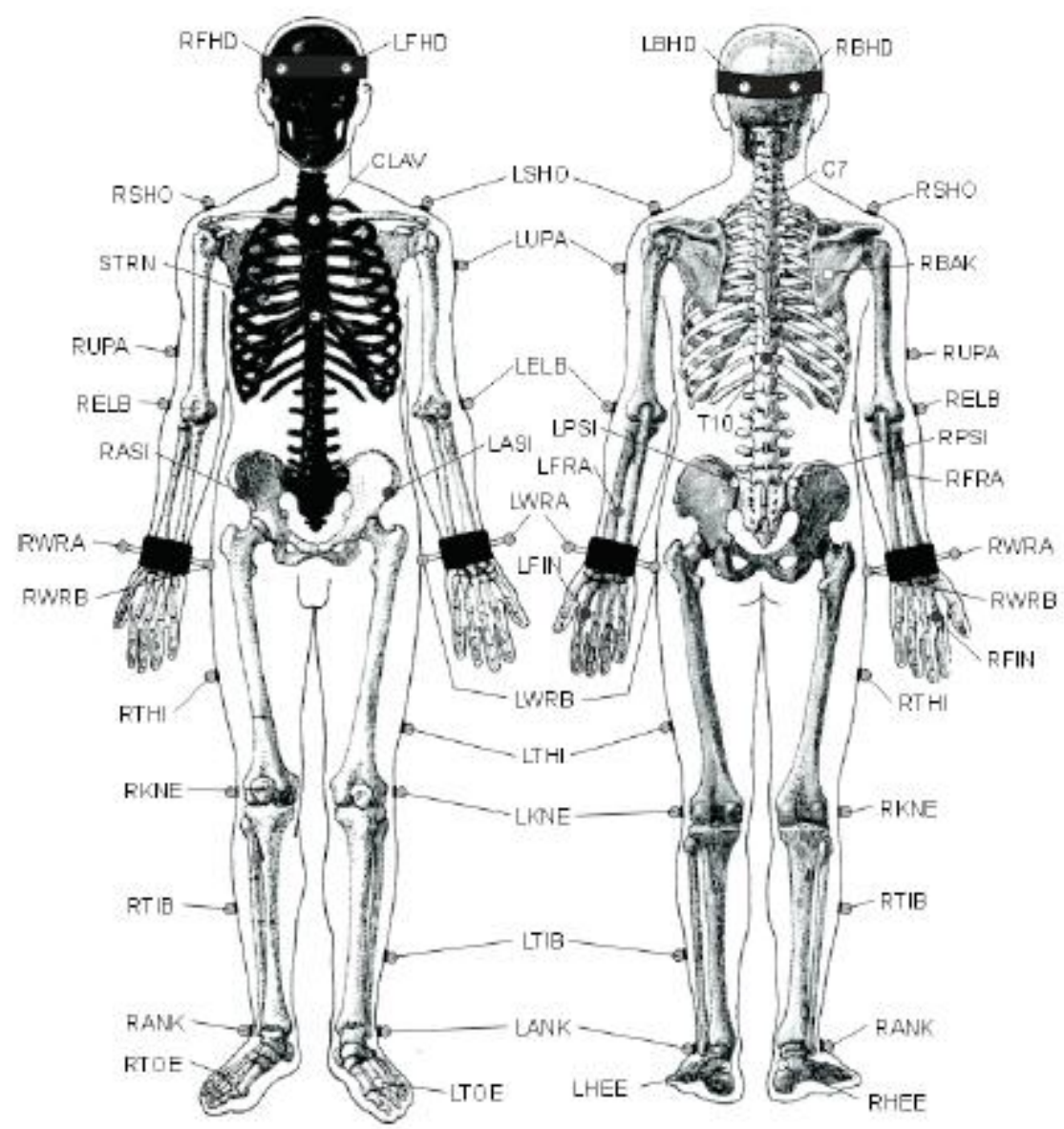

Figure 1. Plug-in-Gait spatial model (Liu, Lian, \& Liu, 2008) for marker placement.

VICON Nexus 1.1 software handled control of cameras and analog data capture and processing. The MX-13 cameras were distributed about subjects and used to capture subject three-dimensional kinematics at $120 \mathrm{~Hz}$. See Figure 2 for marker and recording camera placements.

A JVC TK-C140U Digital Color Video Camera provided video records of subjects performing experimental tasks, with a sagittal view. Two Kistler (Type 9286AA) force plates were used to guide foot placement. The force plates also recorded the ground reaction forces, 
moments, and centers of mass and pressure, but this data was beyond the scope of this study, and will not be discussed.

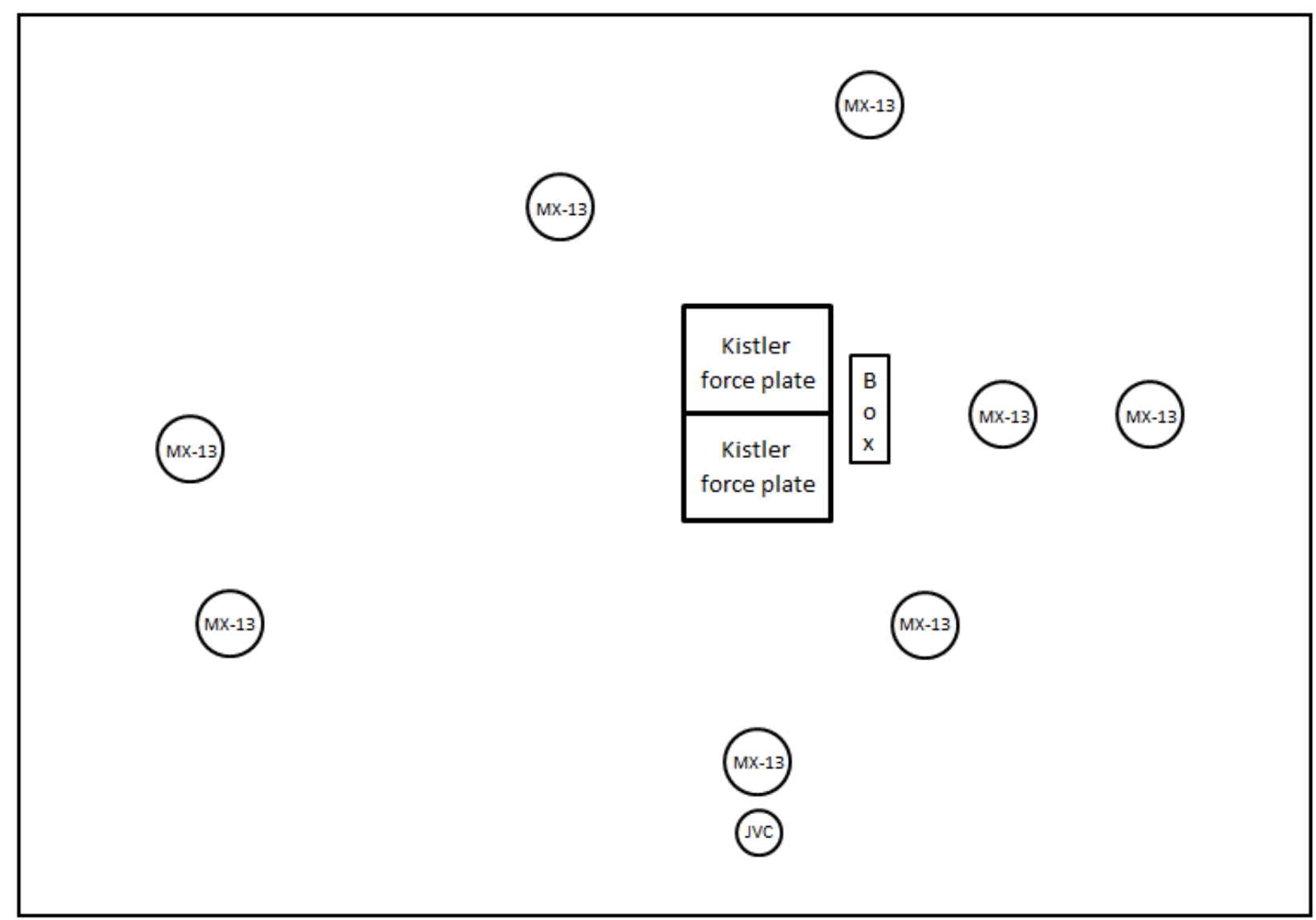

Figure 2. VICON marker and JVC video recording cameras, Kistler force plates, and box placements.

Arm Lifting Station. Two shelves, located $79 \mathrm{~cm}$ and $139 \mathrm{~cm}$ above the floor, were positioned perpendicularly to create a cyclic arm lift workstation. A $9.1 \mathrm{~kg}$ machined box with extended side handles was used for the load for the cyclic arm lifts. Subjects turned $90^{\circ}$ to lift the box from one shelf to the other. A computer program was written to create a lift and lower metronome to control lifting and lowering rates and workloads at six exertions per minute.

Walking Station. A NordicTrack (Model E 3200) treadmill was used to allow subjects to walk at a continuous rate of $4.8 \mathrm{~km} / \mathrm{hr}$ with no inclination. 
Lifting Station. For the floor lift station, subjects were instructed to stand on the force plates, with one foot on each. No lifting technique was specified, to ensure that the method chosen by each of the subjects was not constrained. Listening to instructions that had been previously recorded, subjects were told to pick up the box $(7.3 \mathrm{~kg})$ that was on the floor and bring it up to waist height and hold. When the recording produced a chime, they lowered the box and placed it back on the floor, and returned to a standing position. While at this station, subjects repeated the lifts and lowers until the recording instructed them to proceed to the next station. The box used at this station can be seen in Figure 3 .

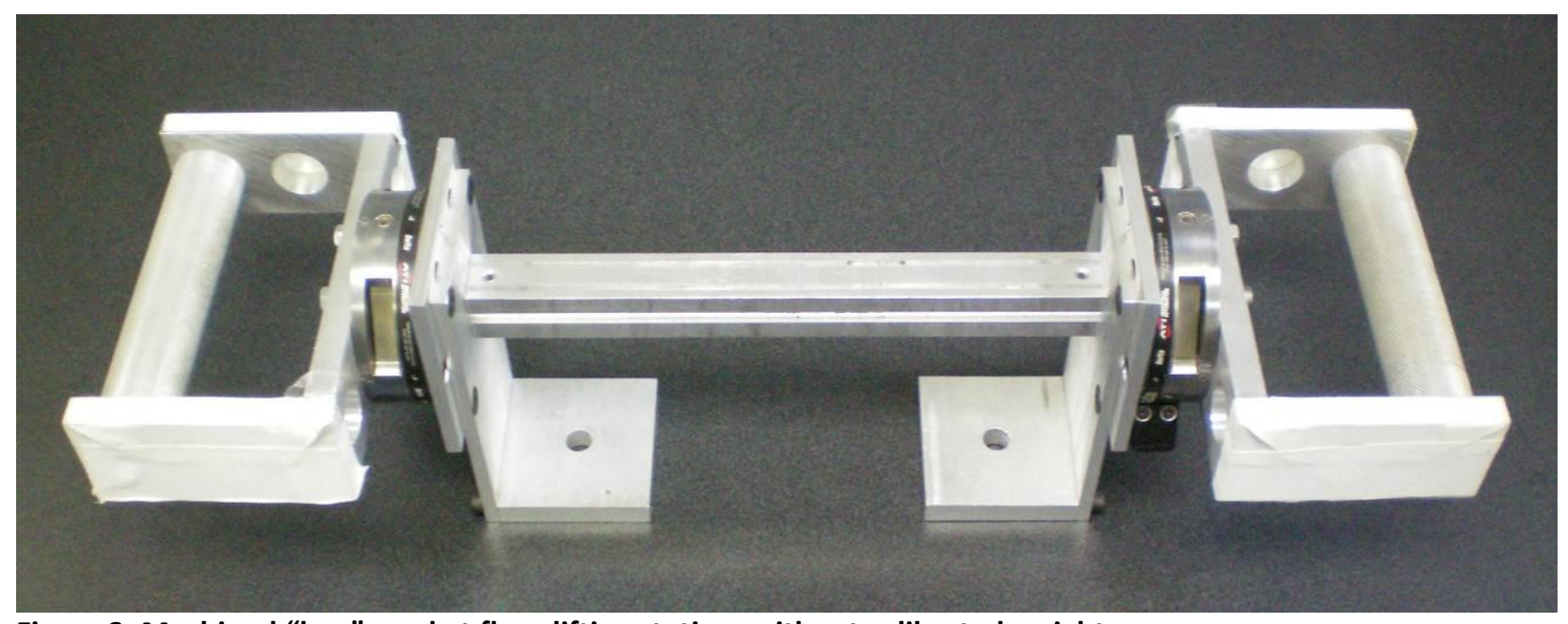

Figure 3. Machined "box" used at floor lifting station, without calibrated weights.

Physiological Recording. A VitalSense ${ }^{\circledast}$ Integrated Physiological Monitoring System, manufactured by Mini Mitter ${ }^{\circledR}$, was used to record skin surface temperatures and body core temperatures using telemetry as described elsewhere (Menze, McMullen, White, \& Dougherty, 1996; Duncan Mitchell \& C. H. Wyndham, 1969; Newsom, et al., 2004; Puhakka, Anttonen, Niskanen, \& Ryhänen, 1994; Ramanathan, 1964). The VitalSense ${ }^{\circledR}$ Monitor functioned at 40.86 $\mathrm{MHz}$ and had transmittals with a Baud rate of $57.6 \mathrm{k}$; Jonah ${ }^{\mathrm{TM}}$ body core temperature oral capsules were swallowed by mouth and VitalSense ${ }^{\circledast}$ dermal patches were placed on each 
subject at the left scapula, left thigh over the rectus femoris, left shank over the tibialis anterior, and right lower arm as shown in Figure 4. Recordings of the four adhesively attached dermal patches were combined to estimate body skin temperature as recommended by Ramanathan (1964) and Mitchell and Wyndham (1969). VitalSense ${ }^{\circledR}$ temperature transmitter capsules were swallowed and dermal thermistor patches were then activated, and individual sensor telemetry transmitter codes were recorded after activation.

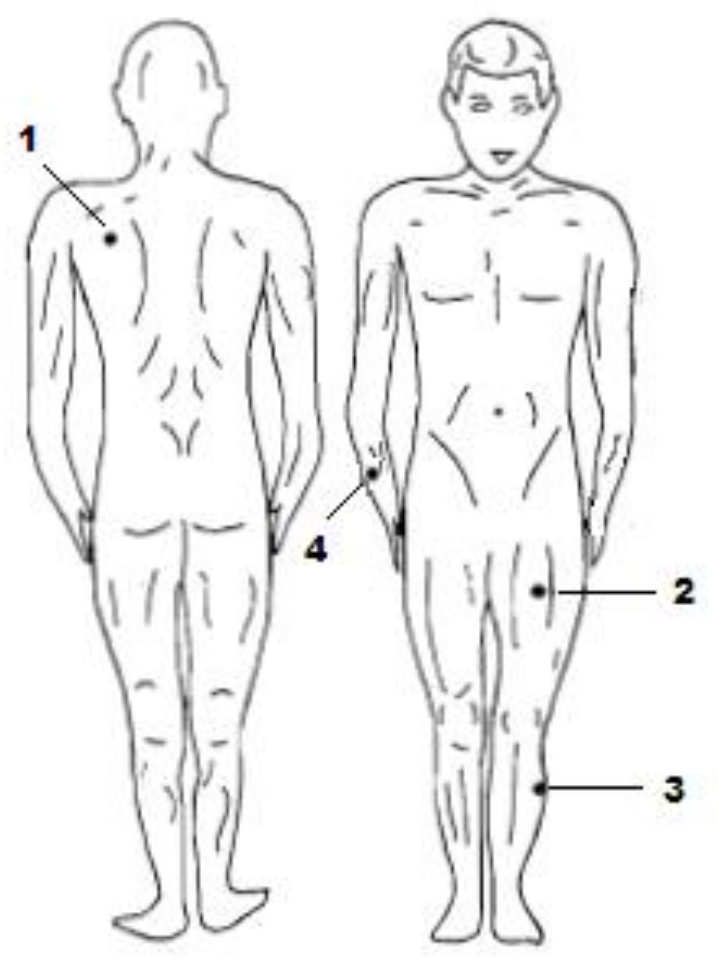

Figure 4. Placement of dermal patches on body at locations of 1) left scapula, 2) left thigh, 3) left shank, and 4) right forearm, modified from model by Mitchell and Wyndham (1969).

Polar $^{\circledast}$ heart rate monitoring telemetry chest bands and receiver wrist watches were used to record subjects' heart rates. Heart rates were averaged over 5-second intervals, and were recorded continuously throughout the experimental period with synchronized timestamped records. The live thermal and heart rate data were used for the termination criteria. The recorded data will not be discussed further, as it was outside of the scope of this research. 
Psychophysical Assessments. Cross-modal matching stimulus sheets were created to allow subjects to create numeric and visual line lengths for gauging systemic fatigue and discomfort, and regional discomfort for body regions shown in Figure 5 (Wiker, Chaffin and Langolf, (1989). Regional ratings were numeric ranging from 0 to 10 , with 0 indicating no sensations of discomfort and 10 being extreme or unbearable discomfort. Global ratings of discomfort and fatigue were gauged on line scales that ranged from "None" (no feeling of discomfort or fatigue) to "Extreme" (extreme or intolerable levels of sensation, forcing cessation of further exposure). Following the guidance of Haagen (1949), the subjects were given only minimum and maximum adjective anchors of "None" and "Extreme", respectively.

Thermal comfort ratings were obtained using a modified Fanger Scale (Fanger, 1967, 1970). The anchor adjectives bounded ratings from "Comfortable" to "Hot." Comfortable was defined as comfortable, while hot was defined as intolerable sensation of body heat leaving one with a sensation of illness and imminent fainting. 

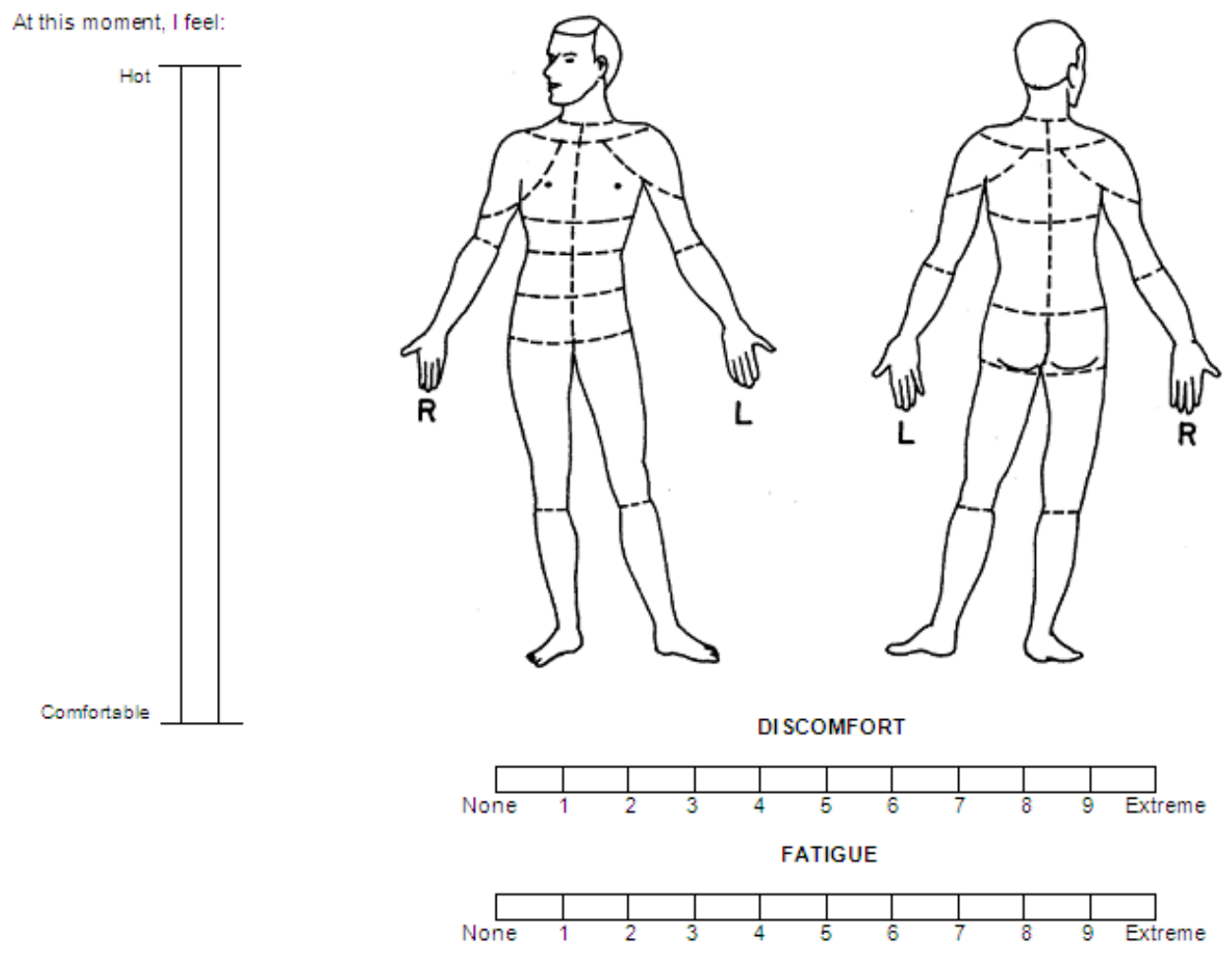

Figure 5. Discomfort, fatigue, and temperature survey administered at questionnaire station, modified from Wiker, Chaffin, and Langolf (1989).

Banding. To simulate the effects of using tape bands that may be used to secure data sensors on a person's body, $5.08 \mathrm{~cm}$ wide polymer Velcro ${ }^{\circledR}$ bands that were coupled using Velcro ${ }^{\circledR}$ fasteners were created. A very thin $(<1 \mathrm{~mm})$ sheet of breathable foam was placed underneath the band to increase surface friction to resist band migration during testing and increase comfort. Bands were applied with bilateral symmetry to the midpoint of the upper arm, thigh, and shank links. Marks were drawn on the subjects' skin, indicating the original location of the bands to ensure that the bands did not migrate during experimental testing.

In order to standardize and control compression pressures of the banding during muscle contraction, a set of 3 small springs were used to fasten the banding. Figure 6 depicts a constructed band and the foam barrier. The springs were produced by Lee Spring Company 
with a spring constant of $0.16 \mathrm{~kg} / \mathrm{cm}$. The springs served to allow expansion of the circumference of the bands without a noticeable material change in the initial contract pressures of bands against the underlying skin.

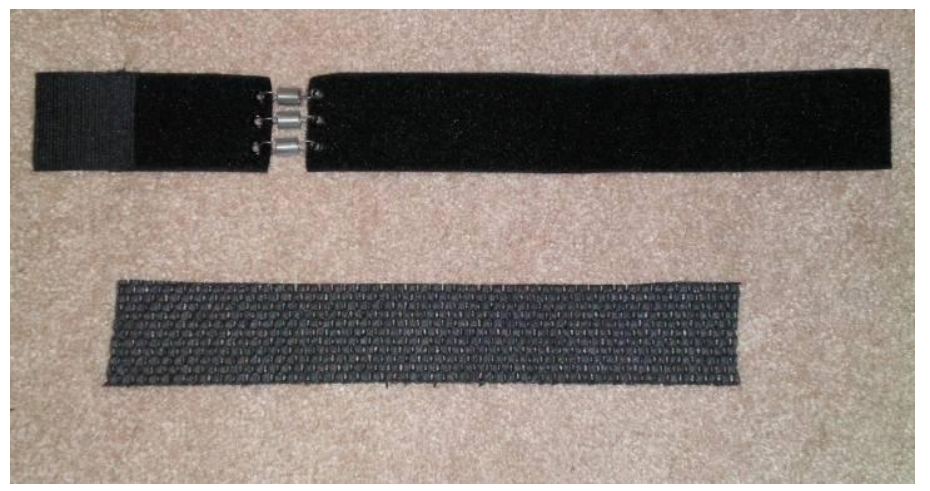

Figure 6. Velcro ${ }^{\circledast}$ band (with springs) and foam barrier from top to bottom, respectively.

Mass Elements. In the high mass experimental condition, small amounts of mass were added to the upper (below the clavicle, approximately over the pectoralis major) and lower (at the waist) regions of the torso and the wrists. Two small metal scuba diving weights of 0.45 and $0.91 \mathrm{~kg}$ were applied bilaterally to the upper and lower torso, respectively, and $0.45 \mathrm{~kg}$ weight bands were added to both wrists, which are shown in Figure 7. Weights coupled to the torso used "suspender" pockets to position the torso masses at the figured regions for each subject's anthropometry. These ensembles were designed to simulate the addition of instrumentation or sensor mass to the body. 


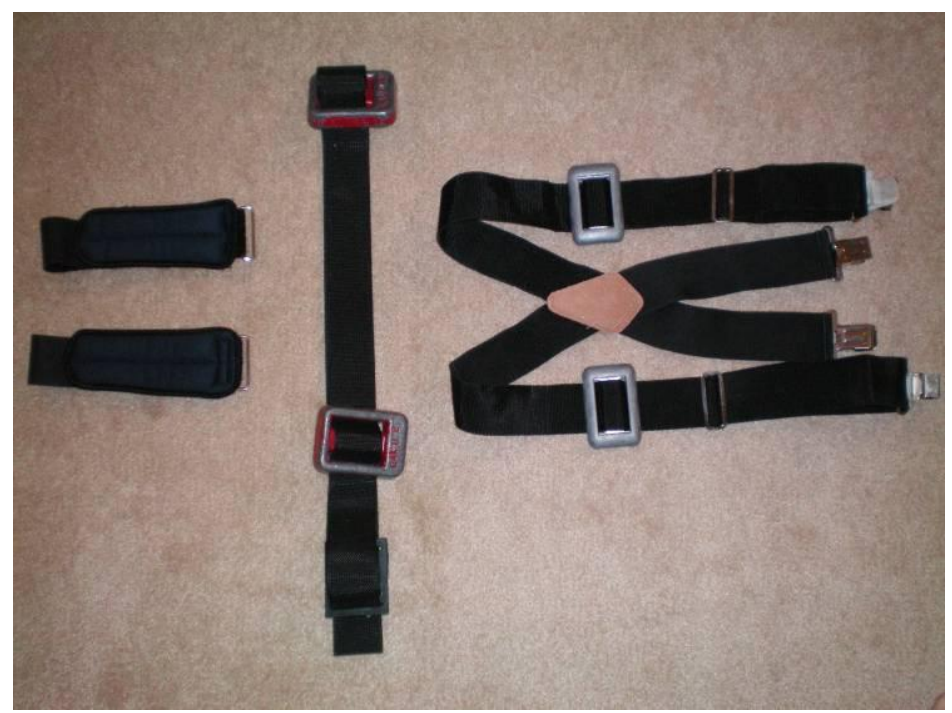

Figure 7. Mass used on wrists, lower torso, and upper torso (from left to right).

Clo Ensembles. The standard amount of insulation required to keep a resting person warm in a windless room at $70^{\circ} \mathrm{F}\left(21.1^{\circ} \mathrm{C}\right)$ is equal to one Clo. The Clo levels were manipulated by changing both the amount of body coverage and thickness of textile material.

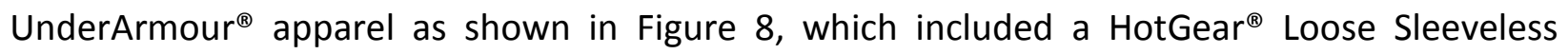
Shooter Shirt (sleeveless shirt) and HotGear ${ }^{\circledR}$ Micro Short (shorts) for the low Clo condition, and a ColdGear ${ }^{\circledR}$ Mock (long sleeved shirt) and ColdGear ${ }^{\circledR}$ Action Legging (long pants) for the high Clo condition. Based on clothing insulation values from ASHRAE Standard 55P, and the adjustment for air flow, the low and high Clo ensembles (including undergarments, socks, and shoes) were estimated to be 0.17 and 0.86 , respectively (ASHRAE, 2003). 


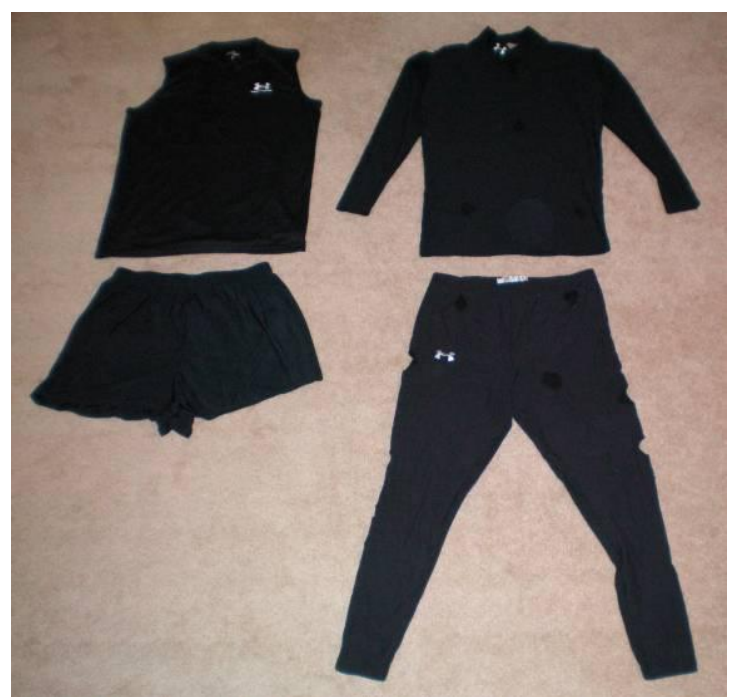

Figure 8. Low Clo (left) and high Clo (right) clothing.

\section{Procedures}

Preliminary Measurements. Upon completion of the informed consent, subjects underwent a series of anthropometric measurements that included body mass, standing height, standing leg length, knee width, ankle width, shoulder offset, elbow width, wrist width, and hand thickness.

Somatotype classification measurements were taken, and included stretch stature, body mass, triceps skinfold, subscapular skinfold, supraspinale (suprailiac) skinfold, medial calf skinfold, biepicondylar breadth of the humerus, biepicondylar breadth of the femur, upper arm girth (with the elbow flexed and tensed), and calf girth, following specific guidance provided elsewhere (Carter, 2002). Each somatotypic measurement was made twice and average values were used. The Heath-Carter (2002) method was used to classify somatotype using combined magnitudes of endomorphy, mesomorphy, and ectomorphy scores. The formulae for computing those classifications are

$$
\text { endomorphy }=-0.7182+0.1451(X)-0.00068\left(X^{2}\right)+0.0000014\left(X^{3}\right)
$$


where $X=$ (sum of triceps, subscapular, and supraspinale skinfolds) multiplied by (170.18/higher in $\mathrm{cm}$ ),

$$
\text { mesomorphy }=0.858 \times \text { humerus breadth }+0.601 \times \text { femur breadth }+0.188 \times
$$
corrected arm girth $+0.161 \times$ corrected calf girth - height $\times 0.131+4.5$

Based upon the height-weight ratio (HWR), one of three equations is used to calculate ectomorphy. If the HWR is greater than or equal to 40.75 , then

$$
\text { ectomorphy }=0.732 \times \mathrm{HWR}-17.63
$$

If the HWR is less that 40.75 , but greater than 38.35 , then

$$
\text { ectomorphy }=0.463 \times \mathrm{HWR}-17.63
$$

If HWR is equal to or less than 38.35 , then

$$
\text { ectomorphy }=0.1
$$

The HWR was computed by dividing the height by the cube root of the mass (Carter, 2002).

Experimental Ensembles. Given the $2^{3}$ factorial design of the experiment, each subject was assigned one of eight experimental ensembles to don, along with a Polar heart rate monitor telemetry band that was placed on the chest just above the xiphoid process, and heart rate receiver watch. If a subject had been assigned an ensemble which included weights, they were put on in the process of changing into the assigned clothing; weights on suspenders were put on after the Polar heart band, underneath the shirt, with the weights positioned under the clavicle yet slightly high on the Pectoralis major.

Weights on the diving belt were placed on the subject after having completely changed into his assigned clothing, and applied on top of the clothing and adjusted to fit around the subject's natural waistline; wrist weights were also applied on top of the clothing, and adjusted to fit around the subject's wrists, but as not to prohibit movement. If the subject had been 
assigned to an ensemble that included bands, they were applied after the subject had changed into his clothing if wearing the low Clo outfit and before if the subject was to wear the high Clo outfit.

Thirty-nine retro-reflective markers were placed on the subject's body using an asymmetric marker set used by the Plug-in-Gait VICON Nexus spatial model, a derivative of the Helen Hayes marker set in which lower extremity wands were absent (Liu, et al., 2008; van Hoof, 2008).

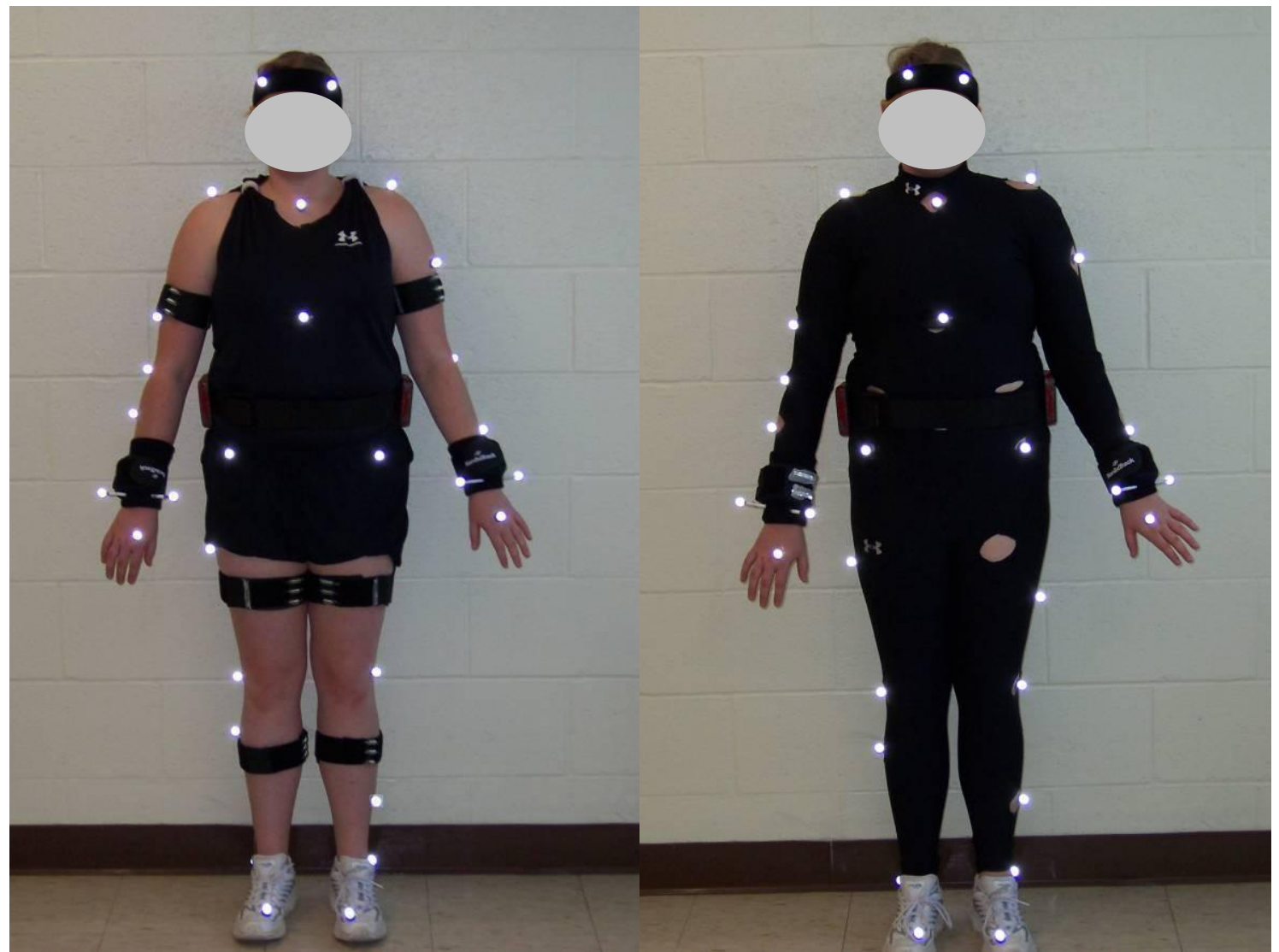

Figure 9. Example of clothing ensembles, from left to right, with subject in low Clo and high Clo with Mass and bands.

Subject Stretch and Warm-Up Protocol. After donning experimental ensembles and testing the telemetry systems, all subjects performed a series of passive stretches demonstrated and guided by a DVD recording of sports therapists demonstrating lower and 
upper extremity, and torso and neck stretch routines that were prescribed for preparation for exercise or physical work. Upon completion of the five-minute stretch, subjects received specific instructions for performing the scheduled whole-body box lifting, standing arm lifting, and treadmill walking tasks, as well as psychophysical and physiological recording and assessment methods that were completed at the Rest/Questionnaire station.

Cyclic Work Protocol. Subjects were asked to perform a two-hour cyclic work protocol that consisted of six twenty-minute cycles, depicted in Figure 10, which consisted of the following:

- Standing and seated rest while completing psychophysical ratings and providing telemetry feeds of body skin and core temperatures and heart rates

- 30 uniformly spaced standing ground-to-waist lifts and lowers

- 30 uniformly spaced standing arm lifts and lowers

- Continuous walking on a treadmill at $4.8 \mathrm{~km} / \mathrm{hr}$ 


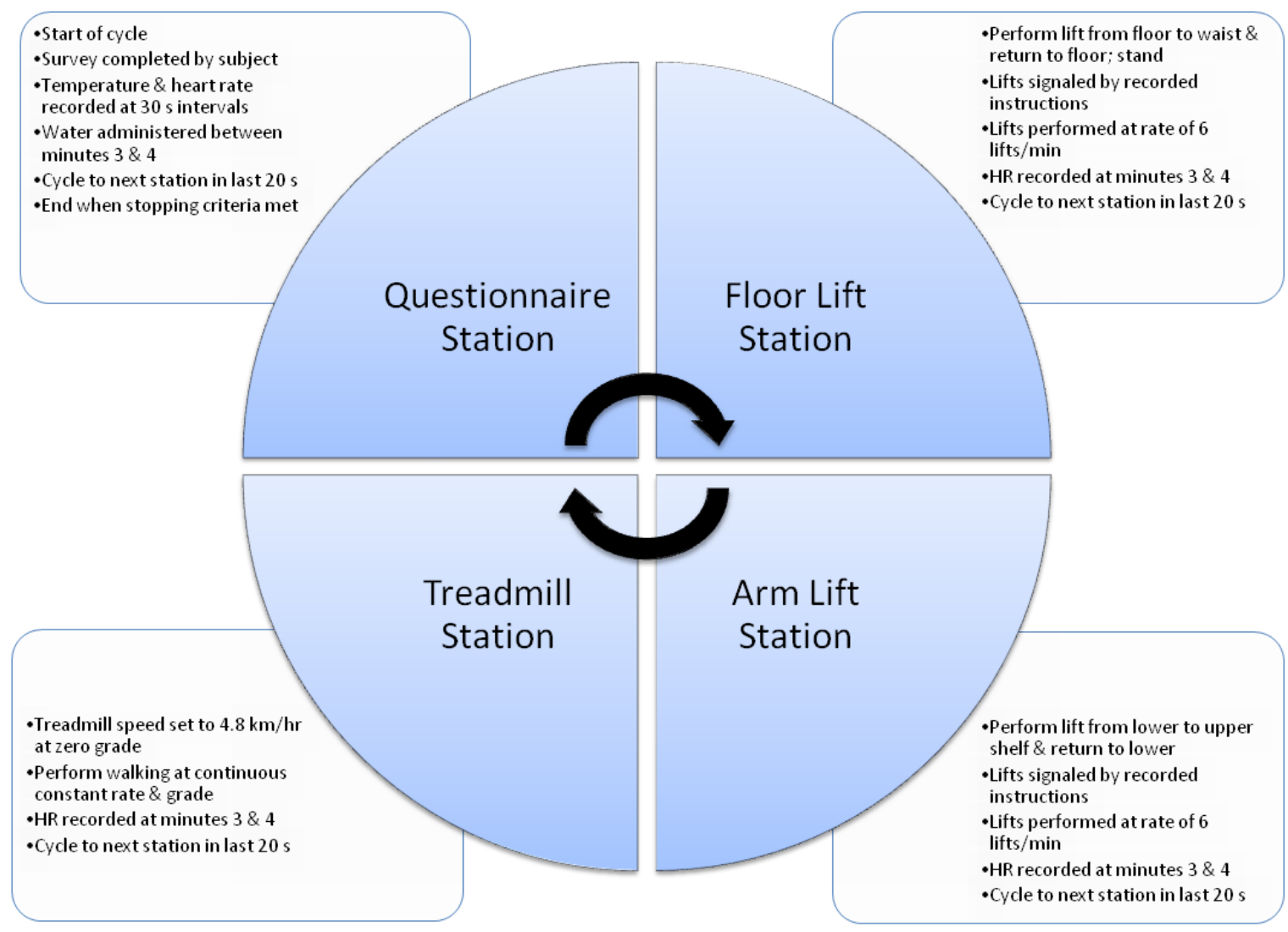

Figure 10. Cyclic work protocol followed for study; the order of arm lift and treadmill stations was interchanged for half of the study.

Subjects were provided with, and drank, a cup of water after providing ratings and telemetry recordings and before the start of the next cycle. Heart rates were visually recorded for subjects at the $3^{\text {rd }}$ and $4^{\text {th }}$ minutes during each 5-minute epoch, while 30 -second samples of body core and skin sensor temperatures were obtained throughout the five-minute rest epoch. The order of standing ground and standing arm lifts was uniformly alternated for all subjects between each 20-minute cycle. Rotation to the next work station occurred during the last 20 seconds of each 5-minute epoch. The combined predicted metabolic energy expenditure for a 20-minute cycle was estimated to be 4.42 and $3.37 \mathrm{Kcal} / \mathrm{min}$ for a $70 \mathrm{Kg}$ male and $50 \mathrm{Kg}$ female, respectively (Garg, Herrin \& Chaffin, 1978). 
Termination Criteria. Subjects stopped testing after completing the sixth complete work cycle after two hours of cyclic work, or when any of the following conditions were exceeded:

- Psychophysical ratings of either fatigue, discomfort, or thermal comfort exceeded $60 \%$ of psychophysical reporting range

- Heart rate exceeded $60 \%$ of the estimated cardiac reserve based upon resting and estimated maximum heart rates (i.e., Maximum Heart Rate $=220-$ Age)

- $\quad$ Core body temperature was greater than $39^{\circ} \mathrm{C}$.

\section{Data Analysis}

Minitab (version 16) was used to perform the statistical analyses. A backward stepwise regression was performed for each dependent variable to evaluate the significance of the independent variables. The initial model included all of the variables, and the variables were removed beginning with the least significant; the alpha level to remove a variable was set at 0.10. A general linear model (GLM) was then performed with the remaining significant variables. 


\section{CHAPTER 4}

\section{RESULTS}

Due to the physiological limitations of the subjects under the experimental conditions, only 13 (out of 32 ) were able to complete all six cycles. Since subjects did not complete the same number of cycles, only the first and last cycles were analyzed. Additionally, four subjects were unable to complete the study past the first cycle. As this study is principally focused on postural changes over time, these subjects were removed from the dataset, and were not considered in the analysis. Stepwise regression and a general linear model (GLM) were used to perform the statistical analyses (Minitab, version 16). The bilateral angles for the hips, knees, and ankles were the dependent (or response) variables. The independent variables were Subject, Banding, Clo, Mass, Part, Phase, and Cycle.

Main and interaction effects are defined statistically as showing a "significant trend" for $p<0.10$, being "significant" for $p<0.05$, and "highly significant" for $p<0.01$.

\section{Stepwise Regression}

Results for the stepwise regression for the hips (left and right) are provided in Table 3 and Table 4; the results for the left and right knees are in Table 5 and Table 6, and the results for the ankles are given in Table 7 and Table 8. After performing the backward regression, Part and Phase were found to be insignificant for all dependent variables; they were removed from the model, and will not be discussed further. Banding, Clo and Mass were found to be significant and were included in the GLM to investigate their main and interaction effects. Although 
Banding was determined to be insignificant for the knees and ankles, it was significant for the other dependent variables and was retained for the GLM.

Table 3. Stepwise regression for the left hip.

\begin{tabular}{|c|c|c|c|}
\hline Step & 1 & 2 & 3 \\
\hline Constant & 114.5 & 113.4 & 112.2 \\
\hline Subject & -0.851 & -0.848 & -0.849 \\
\hline T-value & -9.96 & -9.95 & -9.97 \\
\hline P-value & 0.000 & 0.000 & 0.000 \\
\hline Banding & 6.4 & 6.5 & 6.5 \\
\hline T-value & 4.18 & 4.23 & 4.24 \\
\hline P-value & 0.000 & 0.000 & 0.000 \\
\hline CLO & 3.4 & 3.4 & 3.4 \\
\hline T-value & 2.21 & 2.19 & 2.19 \\
\hline P-value & 0.027 & 0.029 & 0.029 \\
\hline Mass & 6.3 & 6.3 & 6.2 \\
\hline T-value & 3.79 & 3.77 & 3.75 \\
\hline P-value & 0.000 & 0.000 & 0.000 \\
\hline Cycle & -0.72 & -0.72 & -0.72 \\
\hline T-value & -2.37 & -2.37 & -2.39 \\
\hline P-value & 0.018 & 0.018 & 0.017 \\
\hline Part & -0.54 & & \\
\hline T-value & -0.59 & & \\
\hline P-value & 0.558 & & \\
\hline Phase & -0.52 & -0.53 & \\
\hline T-value & -0.76 & -0.78 & \\
\hline P-value & 0.447 & 0.438 & \\
\hline$S$ & 16.1 & 16.1 & 16.1 \\
\hline R-Sq & 22.92 & 22.86 & 22.76 \\
\hline R-Sq(adj) & 21.7 & 21.82 & 21.89 \\
\hline Mallows Cp & 8.0 & 6.3 & 4.9 \\
\hline
\end{tabular}

Table 4. Stepwise regression for the right hip.

\begin{tabular}{|lrrr|}
\hline Step & $\mathbf{1}$ & $\mathbf{2}$ & $\mathbf{3}$ \\
Constant & 112 & 110.6 & 108.1 \\
\hline \hline Subject & -0.64 & -0.641 & -0.636 \\
T-value & -6.6 & -6.61 & -6.56 \\
P-value & 0.000 & 0.000 & 0.000 \\
\hline Banding & 8.6 & 8.6 & 8.8 \\
T-value & 4.92 & 4.94 & 5.01 \\
P-value & 0.000 & 0.000 & 0.000 \\
\hline CLO & 3.9 & 3.9 & 3.8 \\
T-value & 2.23 & 2.23 & 2.17 \\
P-value & 0.027 & 0.026 & 0.031 \\
\hline Mass & 5.7 & 5.6 & 5.5 \\
T-value & 2.99 & 2.96 & 2.92 \\
P-value & 0.003 & 0.003 & 0.004 \\
\hline Cycle & -1.07 & -1.08 & -1.07 \\
T-value & -3.11 & -3.13 & -3.12 \\
P-value & 0.002 & 0.002 & 0.002 \\
\hline Part & -1.2 & -1.2 & \\
T-value & -1.16 & -1.18 & \\
P-value & 18.5 & 18.3 & 18.3 \\
\hline Phase & 0.247 & 0.239 & \\
T-value & -0.63 & & \\
P-value & -0.8 & & \\
\hline & 0.424 & & \\
\hline S & & & \\
R-Sq & & & \\
R-Sq(adj) & & & \\
Mallows Cp & & & \\
\hline & & & \\
\hline
\end{tabular}


Table 5. Stepwise regression for the left knee.

\begin{tabular}{|c|c|c|c|c|}
\hline Step & 1 & 2 & 3 & 4 \\
\hline Constant & 119.2 & 117.3 & 115.4 & 112 \\
\hline Subject & -1.3 & -1.3 & -1.29 & -1.29 \\
\hline T-value & -6.64 & -6.65 & -6.62 & -6.59 \\
\hline P-value & 0.000 & 0.000 & 0.000 & 0.000 \\
\hline Banding & -3.1 & -3.1 & & \\
\hline T-value & -0.87 & -0.87 & & \\
\hline P-value & 0.382 & 0.386 & & \\
\hline CLO & 13.7 & 13.7 & 13.9 & 13.7 \\
\hline T-value & 3.84 & 3.85 & 3.92 & 3.88 \\
\hline P-value & 0.000 & 0.000 & 0.000 & 0.000 \\
\hline Mass & 15.3 & 15.2 & 14.7 & 14.6 \\
\hline T-value & 3.99 & 3.98 & 3.9 & 3.88 \\
\hline P-value & 0.000 & 0.000 & 0.000 & 0.000 \\
\hline Cycle & -6.06 & -6.07 & -6.08 & -6.08 \\
\hline T-value & -8.72 & -8.74 & -8.76 & -8.76 \\
\hline P-value & 0.000 & 0.000 & 0.000 & 0.000 \\
\hline Part & -1.8 & -1.9 & -1.8 & \\
\hline T-value & -0.87 & -0.88 & -0.83 & \\
\hline P-value & 0.387 & 0.380 & 0.407 & \\
\hline Phase & -0.8 & & & \\
\hline T-value & -0.53 & & & \\
\hline P-value & 0.598 & & & \\
\hline$S$ & 36.9 & 36.9 & 36.9 & 36.8 \\
\hline R-Sq & 23.7 & 23.65 & 23.52 & 23.4 \\
\hline R-Sq(adj) & 22.49 & 22.62 & 22.66 & 22.72 \\
\hline Mallows Cp & 8.0 & 6.3 & 5.0 & 3.7 \\
\hline
\end{tabular}

Table 6. Stepwise regression for the right knee.

\begin{tabular}{|lrrrr|}
\hline Step & $\mathbf{1}$ & $\mathbf{2}$ & $\mathbf{3}$ & $\mathbf{4}$ \\
Constant & 117.3 & 119.3 & 115 & 108.5 \\
\hline \hline Subject & -1.16 & -1.17 & -1.17 & -1.16 \\
T-value & -5.69 & -5.74 & -5.75 & -5.68 \\
P-value & 0.000 & 0.000 & 0.000 & 0.000 \\
\hline Banding & 3.3 & & & \\
T-value & 0.89 & & & \\
P-value & 0.373 & & & \\
\hline CLO & 16.6 & 16.4 & 16.4 & 16.1 \\
T-value & 4.47 & 4.42 & 4.43 & 4.34 \\
P-value & 0.000 & 0.000 & 0.000 & 0.000 \\
\hline Mass & 10.7 & 11.2 & 11 & 10.9 \\
T-value & 2.68 & 2.84 & 2.79 & 2.75 \\
P-value & 0.008 & 0.005 & 0.005 & 0.006 \\
\hline Cycle & -6.38 & -6.37 & -6.38 & -6.38 \\
T-value & -8.8 & -8.79 & -8.81 & -8.78 \\
P-value & 0.000 & 0.000 & 0.000 & 0.000 \\
\hline Part & -3.1 & -3.2 & -3.3 & \\
T-value & -1.41 & -1.47 & -1.49 & \\
P-value & 0.158 & 0.143 & 0.136 & \\
\hline Phase & -1.8 & -1.8 & & \\
T-value & -1.1 & -1.11 & & \\
P-value & 0.272 & 0.266 & & \\
\hline & & & & \\
\hline S & 38.5 & 38.5 & 38.5 & 38.5 \\
R-Sq & 22.73 & 22.6 & 22.38 & 21.99 \\
R-Sq(adj) & 21.52 & 21.55 & 21.51 & 21.29 \\
Mallows Cp & 8.0 & 6.8 & 6.0 & 6.3 \\
\hline & & & & \\
\hline
\end{tabular}


Table 7. Stepwise regression for the left ankle.

\begin{tabular}{|c|c|c|c|c|}
\hline Step & 1 & 2 & 3 & 4 \\
\hline Constant & 39.44 & 38.29 & 36.87 & 35.81 \\
\hline Subject & -0.363 & -0.36 & -0.361 & -0.356 \\
\hline T-value & -4.85 & -4.82 & -4.83 & -4.77 \\
\hline P-value & 0.000 & 0.000 & 0.000 & 0.000 \\
\hline Banding & -2.1 & -2 & -2 & \\
\hline T-value & -1.54 & -1.5 & -1.49 & \\
\hline P-value & 0.124 & 0.133 & 0.138 & \\
\hline CLO & 4.6 & 4.6 & 4.6 & 4.7 \\
\hline T-value & 3.4 & 3.36 & 3.37 & 3.49 \\
\hline P-value & 0.001 & 0.001 & 0.001 & 0.001 \\
\hline Mass & 5 & 4.9 & 4.9 & 4.6 \\
\hline T-value & 3.42 & 3.39 & 3.36 & 3.17 \\
\hline P-value & 0.001 & 0.001 & 0.001 & 0.002 \\
\hline Cycle & -1.98 & -1.98 & -1.99 & -2 \\
\hline T-value & -7.47 & -7.47 & -7.49 & -7.52 \\
\hline P-value & 0.000 & 0.000 & 0.000 & 0.000 \\
\hline Part & -0.58 & & & \\
\hline T-value & -0.72 & & & \\
\hline P-value & 0.473 & & & \\
\hline Phase & -0.58 & -0.58 & & \\
\hline T-value & -0.95 & -0.97 & & \\
\hline P-value & 0.341 & 0.332 & & \\
\hline$S$ & 14.1 & 14.1 & 14.1 & 14.1 \\
\hline $\mathrm{R}-\mathrm{Sq}$ & 17.84 & 17.74 & 17.57 & 17.16 \\
\hline R-Sq(adj) & 16.54 & 16.63 & 16.65 & 16.42 \\
\hline Mallows Cp & 8.0 & 6.5 & 5.5 & 5.7 \\
\hline
\end{tabular}

Table 8. Stepwise regression for the right ankle.

\begin{tabular}{|lrrrr|}
\hline Step & $\mathbf{1}$ & $\mathbf{2}$ & $\mathbf{3}$ & $\mathbf{4}$ \\
Constant & 35.87 & 34.71 & 35.67 & 33.2 \\
\hline \hline Subject & -0.137 & -0.138 & -0.142 & -0.136 \\
T-value & -1.76 & -1.77 & -1.82 & -1.75 \\
P-value & 0.080 & 0.078 & 0.069 & 0.081 \\
\hline Banding & 1.6 & 1.6 & & \\
T-value & 1.13 & 1.14 & & \\
P-value & 0.258 & 0.253 & & \\
\hline CLO & 2.6 & 2.6 & 2.5 & 2.4 \\
T-value & 1.84 & 1.84 & 1.77 & 1.68 \\
P-value & 0.067 & 0.066 & 0.078 & 0.094 \\
\hline Mass & 2.9 & 2.8 & 3.1 & 3 \\
T-value & 1.88 & 1.85 & 2.04 & 1.99 \\
P-value & 0.061 & 0.065 & 0.042 & 0.047 \\
\hline Cycle & -2.09 & -2.09 & -2.08 & -2.08 \\
T-value & -7.55 & -7.57 & -7.55 & -7.52 \\
P-value & 0.000 & 0.000 & 0.000 & 0.000 \\
\hline Part & -1.19 & -1.2 & -1.26 & \\
T-value & -1.41 & -1.43 & -1.5 & \\
P-value & 0.160 & 0.155 & 0.136 & \\
\hline Phase & -0.49 & & & \\
T-value & -0.78 & & & \\
P-value & 0.436 & & & \\
\hline & 13.49 & 13.37 & 13.12 & 12.68 \\
\hline S & 12.13 & 12.2 & 12.14 & 11.9 \\
R-Sq & 6.6 & 5.9 & 6.2 \\
R-Sq(adj) & & & \\
Mallows Cp & & & & \\
\hline & & & & \\
\hline
\end{tabular}




\section{General Linear Model}

After performing the stepwise regression, the final model was established. The independent variables of Subject, Banding, Clo, Mass, and Cycle, as well as interactions, were entered into the GLM. For all joints, Subject and Cycle were found to be highly significant.

\section{Hip}

From the ANOVA tables generated for the GLM (Table 9 and Table 10), Banding and all interactions were found to be significant for both the left and right hips. Mass was found to be statistically significant for only the left hip. The adjusted $R^{2}$ values for the left and right hip were 0.6614 and 0.5906 , respectively.

Table 9. Analysis of Variance for the left hip.

\begin{tabular}{|c|c|c|c|c|c|c|}
\hline Source & DF & Seq SS & Adj SS & Adj MS & F-value & P-value \\
\hline Subject & 19 & 78796.1 & 73518.5 & 3869.4 & 34.59 & 0.000 \\
\hline Banding & 1 & 8284.4 & 847.8 & 847.8 & 7.58 & 0.006 \\
\hline CLO & 1 & 881.2 & 121.8 & 121.8 & 1.09 & 0.297 \\
\hline Mass & 1 & 2884.1 & 336.3 & 336.3 & 3.01 & 0.084 \\
\hline Banding*CLO & 1 & 2416.8 & 3032.0 & 3032.0 & 27.1 & 0.000 \\
\hline Banding*Mass & 1 & 532.5 & 2700.0 & 2700.0 & 24.13 & 0.000 \\
\hline CLO*Mass & 1 & 5179.6 & 5327.5 & 5327.5 & 47.62 & 0.000 \\
\hline Banding*CLO*Mass & 1 & 599.0 & 442.0 & 442.0 & 3.95 & 0.047 \\
\hline Cycle & 1 & 2016.8 & 2016.8 & 2016.8 & 18.03 & 0.000 \\
\hline Error & 424 & 47435.1 & 47435.1 & 111.9 & & \\
\hline Total & 451 & 149025.5 & & & & \\
\hline \multicolumn{7}{|c|}{$S=10.5771 \quad R-S q=68.17 \% \quad R-S q(a d j)=66.14 \%$} \\
\hline
\end{tabular}


Table 10. Analysis of Variance for the right hip.

\begin{tabular}{|c|c|c|c|c|c|c|}
\hline Source & DF & Seq SS & Adj SS & Adj MS & F-value & P-value \\
\hline Subject & 19 & 77317.3 & 71246.5 & 3749.8 & 23.26 & 0.000 \\
\hline Banding & 1 & 11944.7 & 1421.5 & 1421.5 & 8.82 & 0.003 \\
\hline CLO & 1 & 956.8 & 109.7 & 109.7 & 0.68 & 0.410 \\
\hline Mass & 1 & 3772.3 & 317.2 & 317.2 & 1.97 & 0.161 \\
\hline Banding*CLO & 1 & 1525.1 & 3410.7 & 3410.7 & 21.16 & 0.000 \\
\hline Banding*Mass & 1 & 288.4 & 2854.3 & 2854.3 & 17.71 & 0.000 \\
\hline CLO*Mass & 1 & 6206.9 & 6554.5 & 6554.5 & 40.66 & 0.000 \\
\hline Banding*CLO*Mass & 1 & 2654.0 & 2142.8 & 2142.8 & 13.29 & 0.000 \\
\hline Cycle & 1 & 4558.6 & 4558.6 & 4558.6 & 28.28 & 0.000 \\
\hline Error & 424 & 68343.7 & 68343.7 & 161.2 & & \\
\hline Total & 451 & 177567.9 & & & & \\
\hline & $960 R$ & $q=61.51$ & $\% \mathrm{R}-\mathrm{Sq}(\mathrm{a}$ & ) $=59.06 \%$ & & \\
\hline
\end{tabular}

Knee

The main effect of Mass and all two-way and three-way interactions were found to be significant, bilaterally. Clo was significant for the right knee, and demonstrated a significant trend for the left. Banding was significant for the left knee, but insignificant for the right. The adjusted $R^{2}$ values for the left and right knees were 0.62483 and 0.5685 , respectively. The ANOVA tables are provided in Table 11 and Table. 
Table 11. Analysis of Variance for the left knee.

\begin{tabular}{|c|c|c|c|c|c|c|}
\hline Source & DF & Seq SS & Adj SS & Adj MS & F-value & P-value \\
\hline Subject & 19 & 360817.0 & 310398.0 & 16337.0 & 26.44 & 0.000 \\
\hline Banding & 1 & 18.0 & 7730.0 & 7730.0 & 12.51 & 0.000 \\
\hline CLO & 1 & 14994.0 & 2309.0 & 2309.0 & 3.74 & 0.054 \\
\hline Mass & 1 & 12498.0 & 11457.0 & 11457.0 & 18.54 & 0.000 \\
\hline Banding*CLO & 1 & 24417.0 & 5430.0 & 5430.0 & 8.79 & 0.003 \\
\hline Banding*Mass & 1 & 12519.0 & 26514.0 & 26514.0 & 42.92 & 0.000 \\
\hline CLO*Mass & 1 & 6585.0 & 7338.0 & 7338.0 & 11.88 & 0.001 \\
\hline Banding*CLO*Mass & 1 & 10008.0 & 5988.0 & 5988.0 & 9.69 & 0.002 \\
\hline Cycle & 1 & 88423.0 & 88423.0 & 88423.0 & 143.12 & 0.000 \\
\hline Error & 424 & 261954.0 & 261954.0 & 618.0 & & \\
\hline Total & 451 & 792234.0 & & & & \\
\hline \multicolumn{7}{|c|}{$=24.8559 \mathrm{R}-\mathrm{Sq}=66.93 \% \mathrm{R}-\mathrm{Sq}$} \\
\hline
\end{tabular}

Table 12. Analysis of Variance for the right knee.

\begin{tabular}{|c|c|c|c|c|c|c|}
\hline Source & DF & Seq SS & Adj SS & Adj MS & F-value & P-value \\
\hline Subject & 19 & 304765.0 & 275987.0 & 14526.0 & 17.83 & 0.000 \\
\hline Banding & 1 & 4321.0 & 1048.0 & 1048.0 & 1.29 & 0.257 \\
\hline CLO & 1 & 22369.0 & 5542.0 & 5542.0 & 6.8 & 0.009 \\
\hline Mass & 1 & 7352.0 & 5704.0 & 5704.0 & 7 & 0.008 \\
\hline Banding*CLO & 1 & 28241.0 & 4232.0 & 4232.0 & 5.19 & 0.023 \\
\hline Banding*Mass & 1 & 11825.0 & 26051.0 & 26051.0 & 31.98 & 0.000 \\
\hline CLO*Mass & 1 & 4488.0 & 5353.0 & 5353.0 & 6.57 & 0.011 \\
\hline Banding*CLO*Mass & 1 & 19242.0 & 13023.0 & 13023.0 & 15.99 & 0.000 \\
\hline Cycle & 1 & 103442.0 & 103442.0 & 103442.0 & 126.97 & 0.000 \\
\hline Error & 424 & 345423.0 & 345423.0 & 815.0 & & \\
\hline Total & 451 & 851467.0 & & & & \\
\hline & $425 \mathrm{R}$ & $-S q=59.43$ & $\%$ R-Sq(ac & dj) $=56.85 \%$ & & \\
\hline
\end{tabular}

\section{Ankle}

ANOVA tables for the ankles are shown in Table 13 and Table 14. For both ankles, Mass and all two-way interactions were significant. The main interaction of Banding was only found to be significant for the left ankle. The three-way interaction between Banding, Clo, and Mass, 
showed a significant trend for only the right ankle. The adjusted $\mathrm{R}^{2}$ values for the left and right ankle were 0.5954 and 0.4095 , respectively.

Table 13. Analysis of Variance for the left ankle.

\begin{tabular}{|c|c|c|c|c|c|c|}
\hline Source & DF & Seq SS & Adj SS & Adj MS & F-value & P-value \\
\hline Subject & 19 & 50967.4 & 45797.8 & 2410.4 & 25.06 & 0.000 \\
\hline Banding & 1 & 170.2 & 1257.4 & 1257.4 & 13.07 & 0.000 \\
\hline CLO & 1 & 1497.6 & 83.7 & 83.7 & 0.87 & 0.351 \\
\hline Mass & 1 & 1029.9 & 1004.0 & 1004.0 & 10.44 & 0.001 \\
\hline Banding*CLO & 1 & 1287.0 & 740.7 & 740.7 & 7.7 & 0.006 \\
\hline Banding*Mass & 1 & 116.1 & 1128.1 & 1128.1 & 11.73 & 0.001 \\
\hline CLO*Mass & 1 & 2360.8 & 2402.3 & 2402.3 & 24.98 & 0.000 \\
\hline Banding*CLO*Mass & 1 & 66.6 & 1.0 & 1.0 & 0.01 & 0.917 \\
\hline Cycle & 1 & 8943.1 & 8943.1 & 8943.1 & 92.98 & 0.000 \\
\hline Error & 424 & 40783.3 & 40783.3 & 96.2 & & \\
\hline Total & 451 & 107221.8 & & & & \\
\hline & $749 R$ & $q=61.9$ & $\mathrm{R}-\mathrm{Sq}(\mathrm{a}$ & $=59.54 \%$ & & \\
\hline
\end{tabular}

Table 14. Analysis of Variance for the right ankle.

\begin{tabular}{|c|c|c|c|c|c|c|}
\hline Source & DF & Seq SS & Adj SS & Adj MS & F-value & P-value \\
\hline Subject & 19 & 30492.5 & 25901.8 & 1363.3 & 9.43 & 0.000 \\
\hline Banding & 1 & 1194.8 & 28.9 & 28.9 & 0.2 & 0.655 \\
\hline CLO & 1 & 483.3 & 6.7 & 6.7 & 0.05 & 0.830 \\
\hline Mass & 1 & 496.8 & 761.0 & 761.0 & 5.26 & 0.022 \\
\hline Banding*CLO & 1 & 5120.2 & 2294.5 & 2294.5 & 15.87 & 0.000 \\
\hline Banding*Mass & 1 & 77.5 & 879.6 & 879.6 & 6.08 & 0.014 \\
\hline CLO*Mass & 1 & 1209.4 & 1300.8 & 1300.8 & 9 & 0.003 \\
\hline Banding*CLO*Mass & 1 & 812.1 & 449.0 & 449.0 & 3.11 & 0.079 \\
\hline Cycle & 1 & 9224.0 & 9224.0 & 9224.0 & 63.8 & 0.000 \\
\hline Error & 424 & 61296.6 & 61296.6 & 144.6 & & \\
\hline Total & 451 & 110407.2 & & & & \\
\hline \multicolumn{7}{|c|}{$S=12.0236 \quad R-S q=44.48 \% \quad R-S q(a d j)=40.95 \%$} \\
\hline
\end{tabular}




\section{Interaction Plots}

Of the angles studied, the knee angles exhibited the most significant difference between the stoop and squat postures that were utilized during the experiment. For this reason, interaction plots were generated for the bilateral knee angles for each of the ensemble conditions. The main effects, two-way interactions, and three-way interactions are shown in Figure 11, Figure 12, and Figure 13, respectively. Lower values for the angles indicate a more stooped (straight-legged) posture, while higher values indicate a squatting (bent-knee) posture. Additionally, a steeper slope in the interaction plots indicates a greater deviation between the originally chosen lifting strategy and the lifting strategy used in the last experimental cycle.

For the main effects (Figure 11), Banding had smaller initial values with less of a slope, compared to the low condition, meaning that the initial posture was less of a squat and transitioned less. There also was only a slight difference in the average starting joint angles and slope for Clo. The addition of mass resulted in more of a squat posture for the first cycle, with a more pronounced transition to a more stooped posture for the last cycle. 


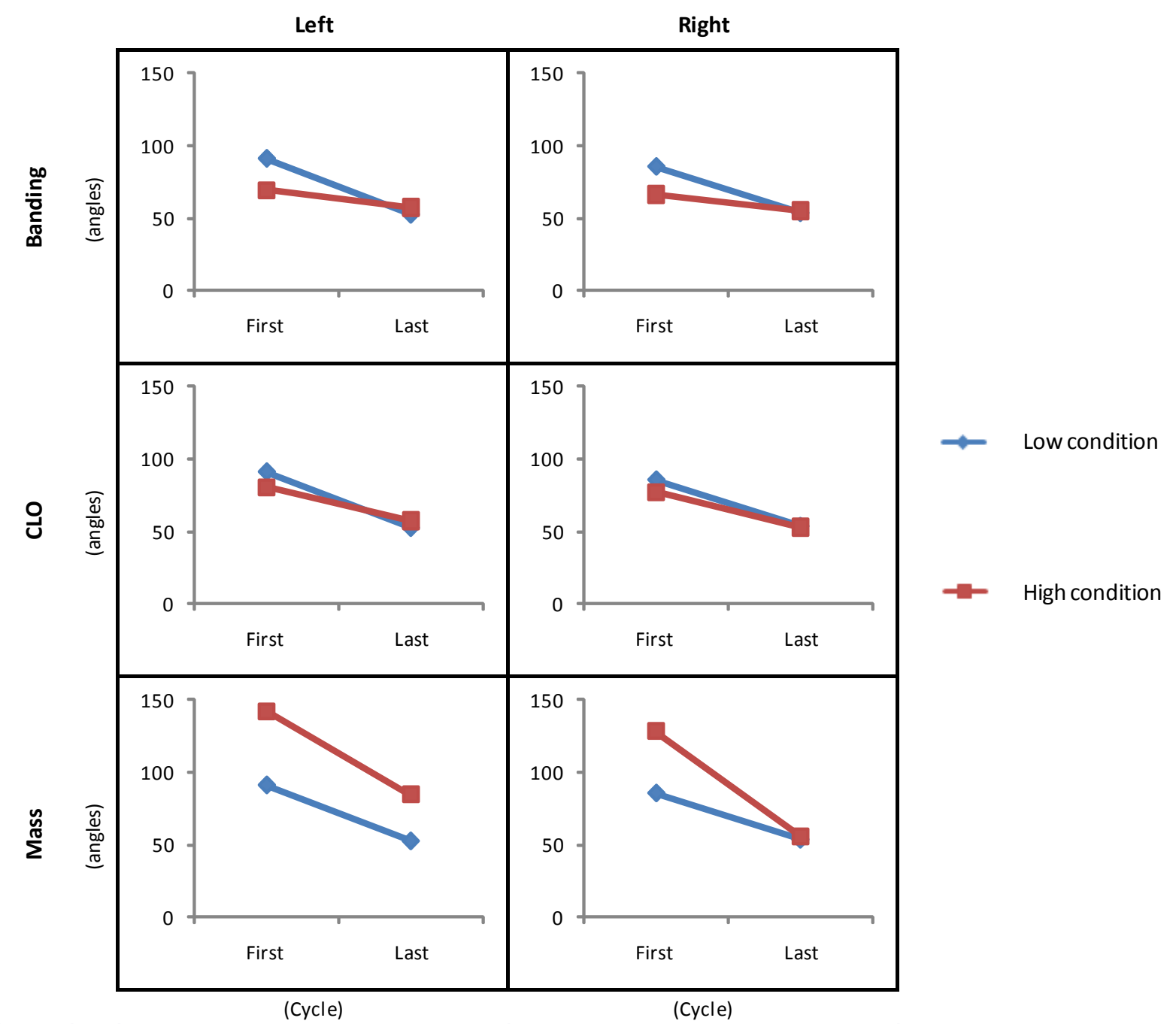

Figure 11. Interaction plots for main effects for the left and right knees.

For the two-way interactions (Figure 12), Banding and Clo exhibited a similar transition from the first cycle to the last, but with more of a squat than the low condition. The Banding and Mass interaction was similar for the high and low conditions, with only a slight difference in slope and less of a stoop posture for the left knee in the last cycle. The interaction between Clo and Mass indicated more of a squat from the first cycle, with a more significant transition to a stooped posture. 


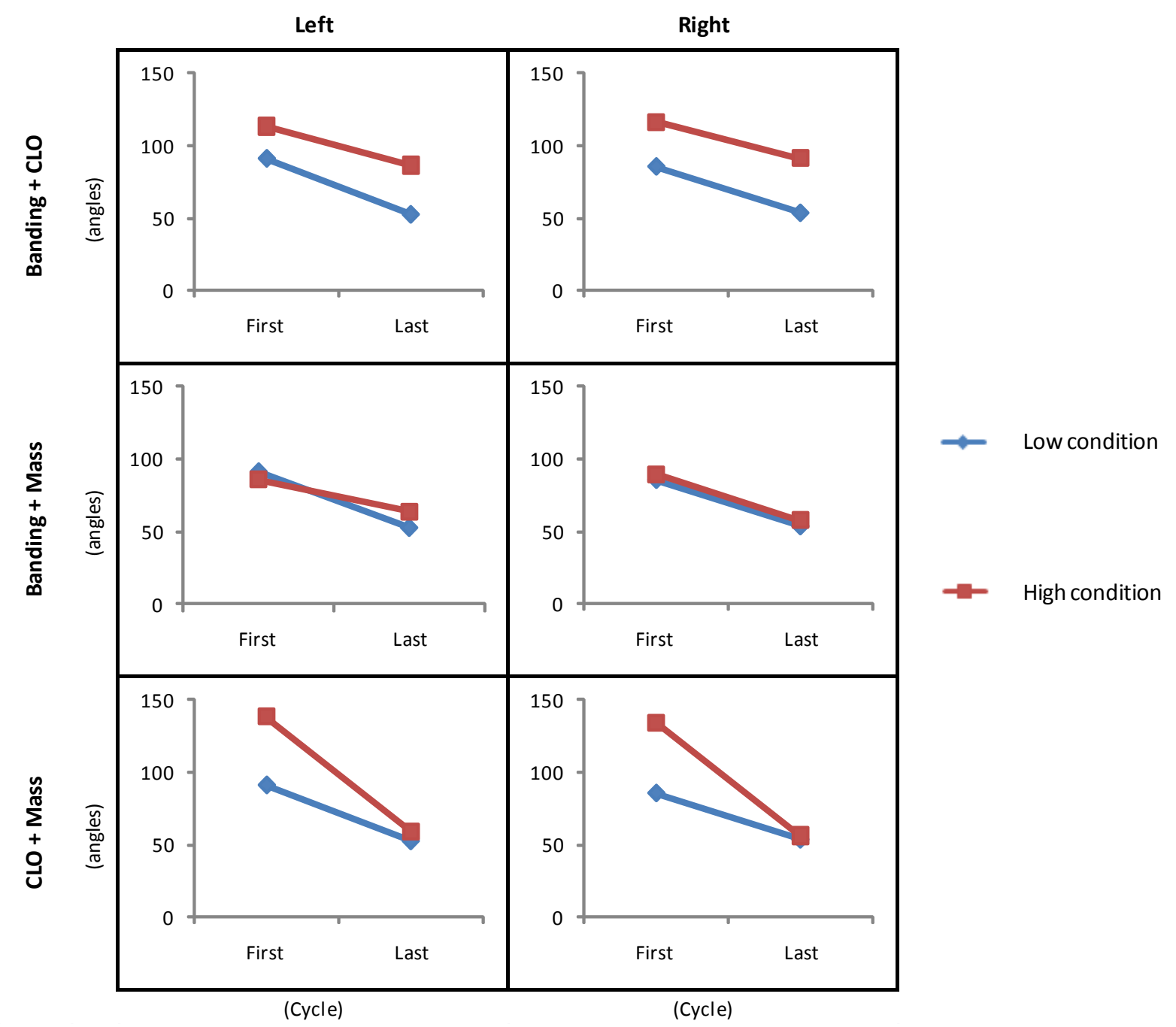

Figure 12. Interaction plots for two-way interactions for the left and right knees.

In comparison to the previous plots, the three-way interaction (Figure 13) between Banding, Clo, and Mass shows that a stoop was adopted for the first cycle, with a slower change in posture to the last cycle. 


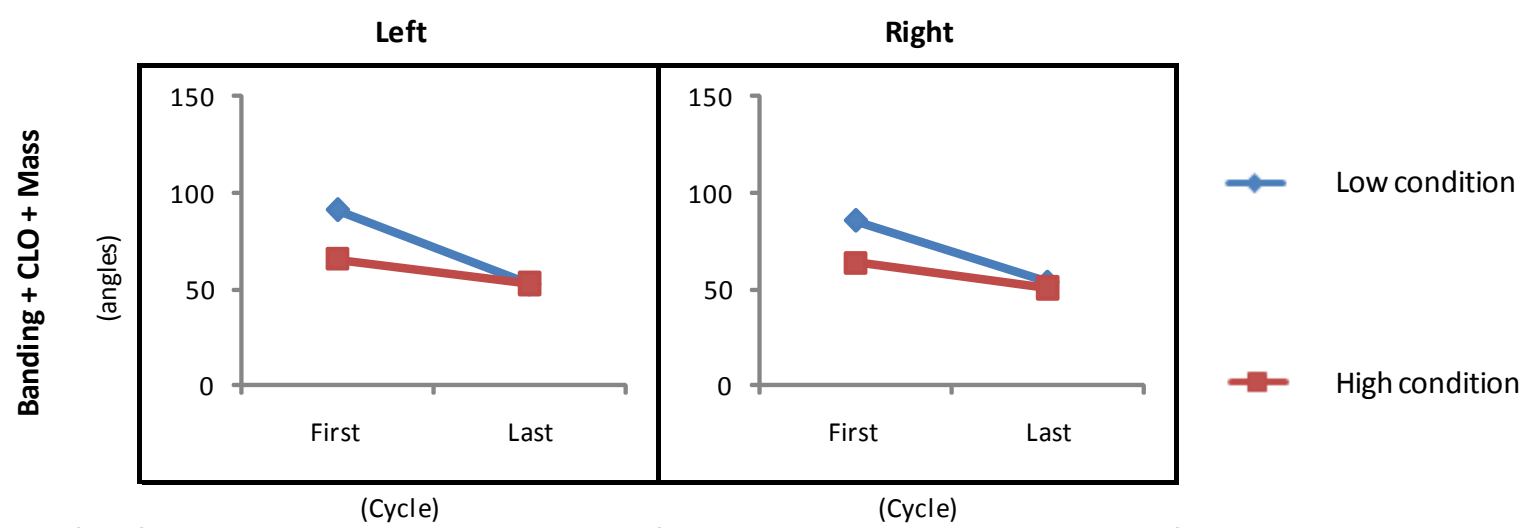

Figure 13. Interaction plots for three-way interactions for the left and right knees. 


\section{CHAPTER 5}

\section{DISCUSSION AND CONCLUSIONS}

The purpose of this study was to determine whether or not the addition of various elements, namely those of Clo, Banding, and Mass, would affect the postures associated with performing repetitive lifting tasks. While the study was designed as a full-factorial, between subjects design, subject attrition led to data loss and an unbalanced design. It was intended that the subjects complete six cycles; however, due to testing termination criteria, only 13 of the 32 subjects were able finish, with 4 subjects unable to complete the study past the first cycle. The stepwise regression identified insignificant independent variables to be removed before entering into the GLM. Angles of the hips, knees, and ankles were analyzed, as these joints provide a clear representation of the posture of the lower body in performing the floor to waist lift.

After running the GLM, the adjusted $R^{2}$ values indicated that a good fit existed between the variables in the model. For the bilateral joints of the hips, knees and ankles, the adjusted $\mathrm{R}^{2}$ values were 0.6614 and $0.5906,0.62483$ and 0.5685 , and 0.5954 and 0.4095 , respectively. Despite the highly significant inter-subject variability, these adjusted coefficients of determination adequately explain the variation of the model for each of the segments.

The main effect of Clo was found to be insignificant for the hip and ankle angles, with a significant trend for the left knee, and Banding insignificant for the right knee and ankle. A reason for the difference in the significance for the knee and ankle joints between Clo and Banding could be due to subjects standing on their toes. In order to complete a lift using the 
squat technique, subjects would most likely have their heels off of the ground to maintain balance during a low squat. Conversely, if subjects were using a stoop technique, their heels would have been touching the floor. In either posture, the angles generated would have been approximately the same, and hence an insignificant effect. With Banding having an insignificant effect on the right knee and ankle angles, it is possible that it had an effect similar to that of a compressive stocking, in which the ability to reduce discomfort and fatigue in the legs can be achieved by wearing compressive stockings (Kraemer, et al., 2000). Consequently, the Banding applied in this study could have created similar responses, preventing the subjects from fatiguing, which would explain why there was no difference in these angles over time. Instead of the Banding causing discomfort to the subjects, it is possible that the Banding actually reduced the onset of fatigue. It was hypothesized that the addition of Banding (as well as the other elements) would cause the subjects to fatigue and change posture over time. Therefore, individuals exposed to this variable would not have needed to change their posture over time. Conversely, with Banding exhibiting a highly significant effect for the hips, this could be explained by effects commonly seen when pressure bandages have been applied. Bandages of this type are capable of causing pain or discomfort to the individual (Botti, et al., 1998). The Banding in this study could have aliased these effects as well, thereby accounting for its significance for the hip angles. In order to compensate for this localized discomfort, the subjects would have adjusted their lifting style.

Mass displayed a significant effect for all joints except for the hips. The left hip displayed a significant trend $(p=0.084)$, while the right hip was insignificant $(p=0.161)$. While some of the subjects may have had prior experience in performing repetitive lifting tasks, it is highly unlikely 
that they added significant mass to their bodies during their previous lifting experiences. More effort would be required to perform the lifts with the Mass, so it is reasonable that the increased mass affects lifting posture over the course of the completed cycles. This is further supported in the interaction plots (Figure 11), where there was a steeper slope for the high condition, meaning a quicker transition in posture. The plots also demonstrated that subjects with the Mass started in more of a squat posture than the low condition, meaning that the location of the Mass on the body may also have had an impact on their lifting strategy. With some of the Mass being placed on the upper torso, leaning over in a stoop may have caused pain in the back.

Mass was found to have a significant effect on the ankle angles, in contrast to Banding (right ankle) and Clo, which were not significant. This could be explained by the idea that subjects wearing the added mass may be less likely to lower all the way to the ground in a squat. With Mass added to the upper body, it may not have been the initial lowering to the ground that the subjects found difficult, as much as rising. Not only were the subjects lifting the box, but they would also have to lift themselves, which would be inhibited by additional weight. Subjects with weaker leg muscles would have found this even more challenging, which would have led these individuals to modify their lifting technique even sooner, or drop out of the study due to fatigue. Overall, the added mass made the task more metabolically demanding, causing those subjects to fatigue more rapidly. Hence, the onset of fatigue and change in lifting posture resulted in a difference in the means between the Mass and no Mass conditions for all of the joints. The location of the mass placed on the subjects may also have been a key component in the onset of fatigue. If mass were to be placed closer to the core of the body, the 
task may have been less fatiguing. It is important to note that the four subjects who were unable to continue past the first cycle were all exposed to an ensemble with a high Mass condition. This further supports that mass is a confounding variable.

All two-way interactions between Banding, Clo, and Mass were significant for the hip, knee, and ankle angles. The significance is also supported by the interaction plots for the knees (Figure 12), with the most dramatic results being visible for the Banding and Clo, and Clo and Mass interactions. Since each of the individual components had a significant effect on the angles for at least one of the joints, these interactions demonstrate a synergistic effect of the independent variables on posture.

The three-way interaction between Banding, Clo, and Mass was significant at the hips and knees, with a significant trend for the right ankle. Interaction plots for the knee joints (Figure 13) also support this significance, showing that subjects demonstrated a change in posture from the first to the last cycle, with less of a squat in the first cycle when compared to the low condition. This difference between the high and low conditions further indicates that the added ensemble components affected the lifting strategies of those subjects. The significance of this interaction, particularly for the knees, would be able to support the theory of subjects transitioning from a squat to stoop in performing the lifts. This is supported in Figure 14 and Figure 15, where the subject started with a squat lift, and was performing stoop lifts in his last cycle. While lifting by means of a squat, the knee angles would have a noticeable change from that of standing. However, once a stoop was utilized, there would be little to no distinction between standing and lifting. Thus, the significance of this not only demonstrates a 
change in posture over time, but specifically that some subjects transitioned from a squat to stoop. There is no evidence to suggest that any subjects transitioned from a stoop posture to a squat posture during the experiment.

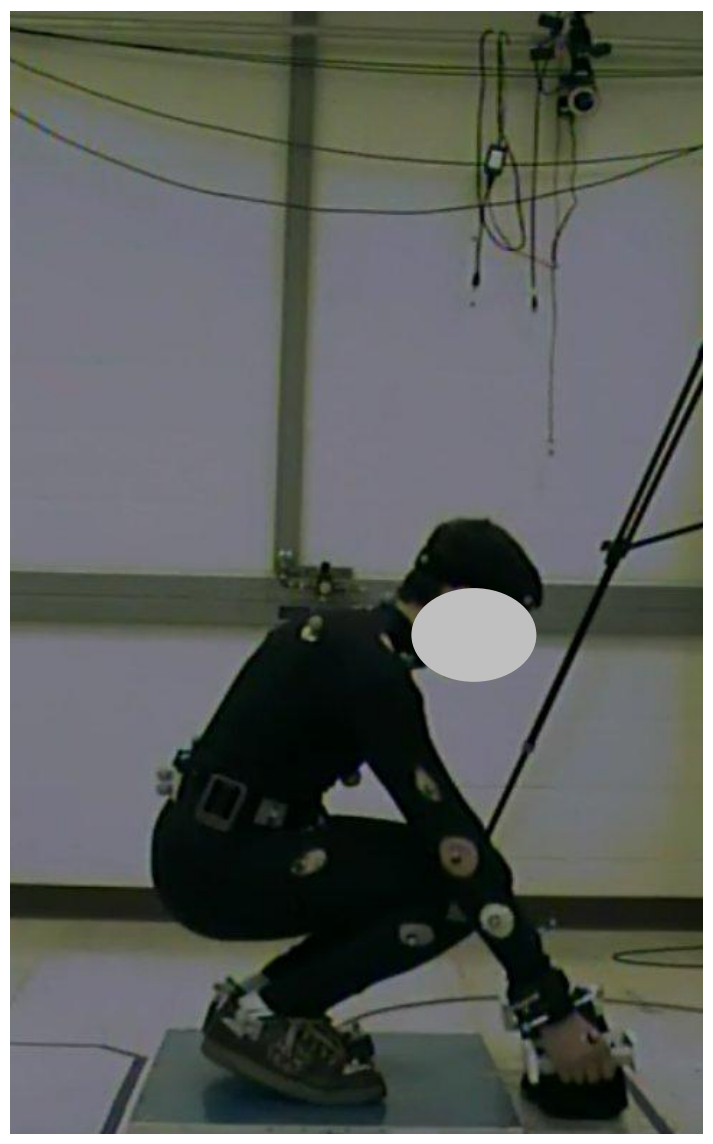

Figure 14. Subject 16 in squat for Cycle 1.

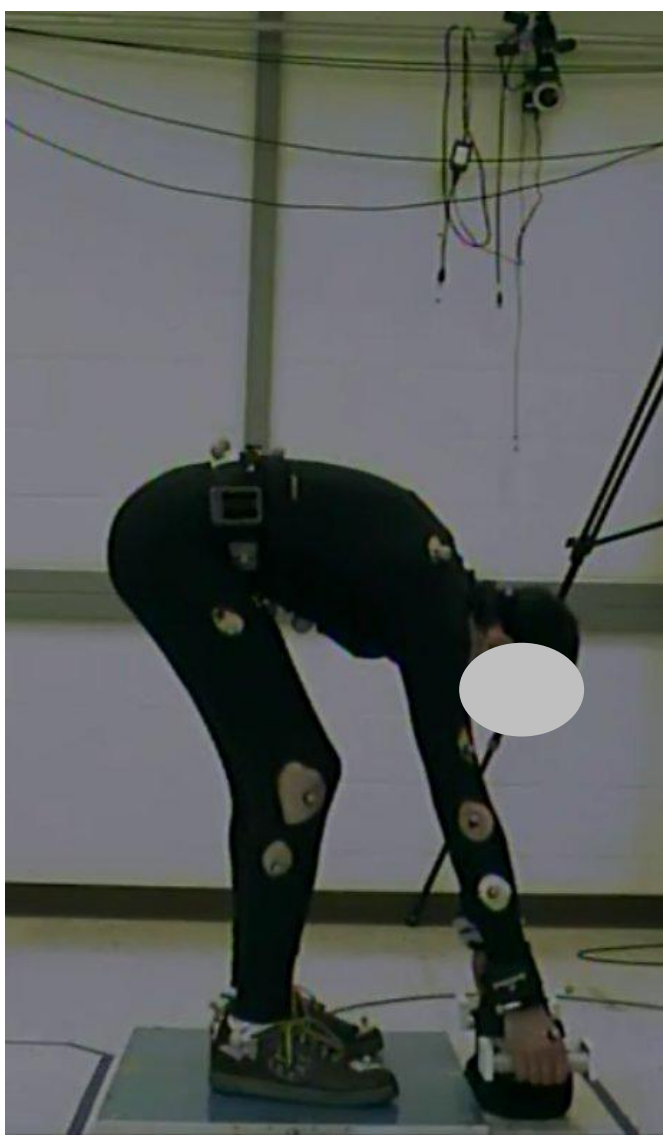

Figure 15. Subject 16 in stoop for last cycle (Cycle 3).

For those instances where the significance of a main or interaction effect was not consistent bilaterally for a joint, it is possible that an individual could have adopted a hybrid posture. Instead of strictly performing either a stoop or squat, a subject may have adopted a modified posture in order to complete the lifting task. Such a posture could be achieved by moving the feet so that they would no longer be placed together and side by side. Subjects could have widened their stance, or even staggered it (having one leg slightly behind the other). 
In the instructions given to the subjects, they were only told to lift the box from the floor, hold it at the waist, and then return it to the floor, following the recorded instructions. No technique was specified as to how to perform the lift, ensuring that the lifting technique chosen by each of the subjects was not constrained. An instance of this posture is shown in Figure 16. It is also feasible that in doing so, they could have leaned forward more in order to perform the lift. By doing this, the individual would not only be changing his lifting strategy, but also adjusting his posture to compensate for any discomfort or fatigue associated with the ensemble.

If the subject combined several of the mentioned strategies, it would be possible to maintain the same lower body posture for one side of the body, but not the other. For example, if the subject began the study by performing a squat lift, he might transition to putting one leg slightly behind the other, instead of moving into a stoop lift. In doing this, it would be probable that he would be able to maneuver the motion of a squat in one leg, but simultaneously maintain a straight-legged posture in the other. As a result, one leg would exhibit no change in posture, while the other would indicate otherwise. Hence, this could be used in explaining why a variable was shown to be significant for the right and not the left, or vice versa. Another explanation could be the asymmetrical design of the Plug-in-Gait model for marker placement. With this model, markers were not placed in the same locations bilaterally, meaning that they were not always placed on the same muscles. With the muscles activating differently for a given posture, it is possible that there was variation in the marker movement. 


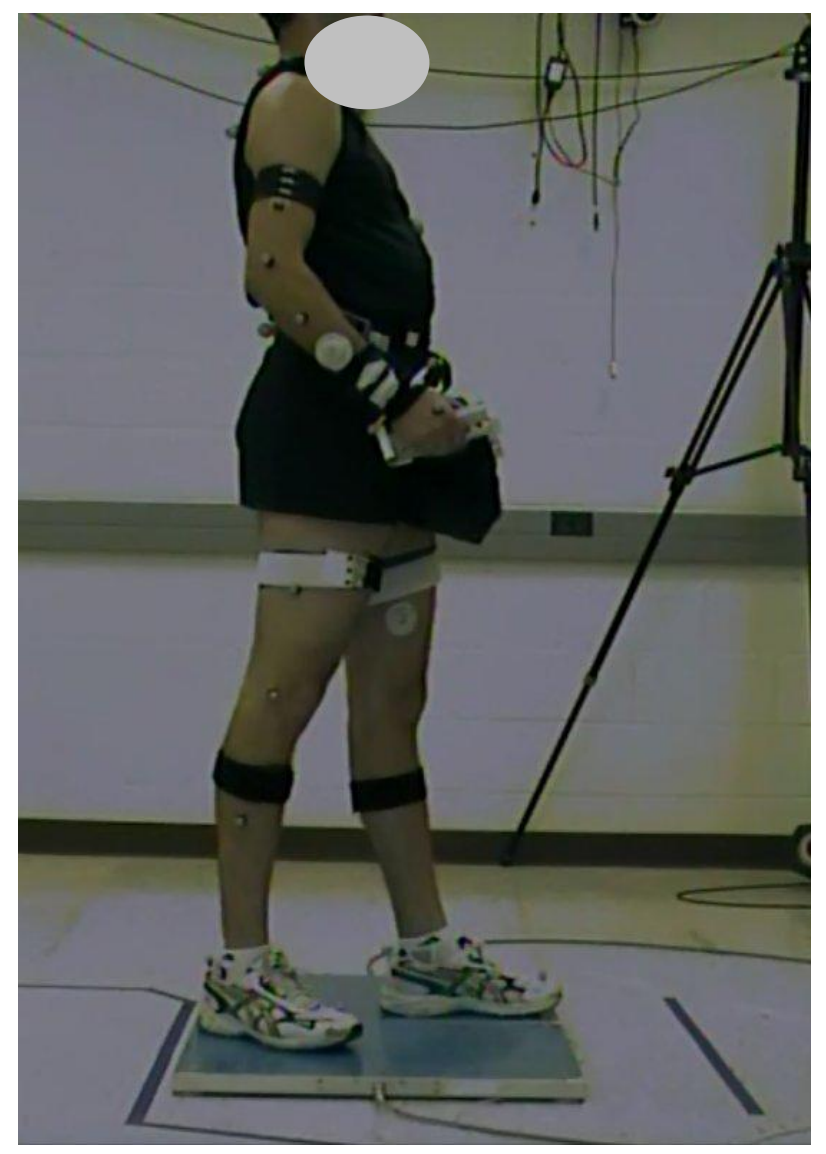

Figure 16. Staggered foot posture adopted by Subject 5.

Cycle and Subject were significant for all joints under consideration. With Cycle having a significant effect, the hypothesis that subjects exposed to the high ensembles would fatigue over time was supported. While the relationship between the first few cycles may have been insignificant, there was significance between the first cycle and the last cycle completed by the subjects, revealing that task fatigue significantly affects how the tasks are performed, and that additional constraints on the body, such as Banding, Mass, and Clo may adversely affect the subjects' endurance. This relationship was further supported by the interaction plots generated for the knees, showing that systemic fatigue occurred with all ensembles. The hypothesis was also able to be supported by these plots, since the angles produced during the first and last cycles for the high ensemble conditions were different from those of the all low condition. 
Since a between-subjects design was used for this study, it was not surprising for Subject to be a significant variable for all bilateral joint angles. Both men and women participated in the study, which was further diversified by age and body types. Each person also had different tolerance thresholds, and while one may have been able to withstand more fatigue and discomfort, a different individual may have perceived that same level as being intolerable. This alone would have caused a difference between the number of cycles completed by one subject, versus that of another. Physical fitness also varied among the subjects, as some could perform the assigned tasks with ease while others visibly struggled.

Recommendations for future studies would be to include additional populations, based on fitness and experience, such as athletes and industrial workers. With subjects reaching the termination criteria within only a few cycles, it brought into consideration that these individuals may be lacking in physical fitness. Changes to the criteria, within safe limits, may have been necessary to extend the number of cycles that the subjects were capable of completing. By recruiting subjects that would be more physically capable of performing the tasks, it is likely that they would be able to survive all six cycles. Furthermore, if more of these subjects were attained, there would be a larger sample size. It is also suggested that, in the future, subjects be chosen based upon a set of physical standards; this could be done by first having the subjects complete several exercises, and rate their performance. Besides looking at a fit population, another that may be valuable to consider is that of experienced individuals. This would consist of individuals who utilize Clo, Banding, and Mass outside of the scope of study, and are exposed to these conditions on a regular basis (e.g., construction workers, electricians, miners, etc.). By exposing individuals who regularly wear ensembles similar to the test conditions, it would be 
feasible to observe a higher survival rate, as well as possibly unmodified postures. If an experienced individual were exposed to the ensemble with all high variables, it is conceivable that he has already learned to adapt to the conditions, and would therefore not exhibit any significant changes between cycles.

Another suggestion for future research would be to utilize a within-subjects design. For this study, a between-subjects design was used, which exposed subjects to only one ensemble. If the same protocol was followed, but instead using a within-subjects design, each subject would be exposed to all ensembles. While a disadvantage to this approach would be that subjects could learn strategies between each of the tests, it would be beneficial to see the results for one person for each of the eight ensembles. This would also result in the variability between the subjects being eliminated. Although similar results would be expected, in that Clo, Banding, and Mass would have significant effects on the postures, it may be desirable to see how these changes occur for each individual. A further recommendation for future research would be to perform the same protocol without the added ensembles. It would also be beneficial to decrease the metabolic demand of the tasks being performed (i.e., box mass, number of lifts). From this study, the onset of fatigue was found to be a major factor. It would be beneficial to see how overall systemic fatigue occurs without placing these physical burdens on the subjects.

For those looking to venture into analyzing repetitive tasks using an on-body sensor suit, it is important to consider that any equipment placed on the workers may affect their posture. If additional clothing or elements are included in an individual's attire, the postures desired to 
be studied are expected to deviate from individual's preference, as well as change over time due to increased levels of fatigue. Despite the significance in the independent variables in this experiment, fatigue was a significant factor in the experiment due to the intense physiological demands placed on the subjects. The findings of this study demonstrated that workers adjusted their movement strategies over the course of the experiment. It is important to note that the findings reported here are only immediately applicable to the ensemble conditions tested and limited in extrapolation by the uncontrolled environmental conditions of the laboratory. Despite the limitations discussed herein, the author contends that the results are valid to the extent that the experimental methods and conditions are representative of actual work environments. It is recommended that companies consider the aforementioned effects prior to placing additional physical burdens on their workers. Based on the findings of this study, it is suggested that if battery packs, bandaging to secure sensors, and clothing are the only options available to perform a study, that they be used with caution. 


\section{REFERENCES}

Abitbol, M. M. (1988). Effect of posture and locomotion on energy expenditure. American Journal of Physical Anthropology, 77(2), 191-199.

ASHRAE. (2003). Thermal Environmental Conditions for Human Occupancy, BSR/ASHRAE Standard 55P (pp. 33-39). Atlanta, Georgia.

Bale, S., \& Harding, K. (1985). Accident and emergency: Dressing down the discomfort. Nursing Times, 81(39), 42.

Balogun, J. A. (1988). Prediction of energy expenditure during load carriage on the head and by yoke. Indian Journal of Medical Sciences, 42(10), 235-241.

Bell, A. L., Pedersen, D. R., \& Brand, R. A. (1990). A comparison of the accuracy of several hip center location prediction methods. Journal of Biomechanics, 23(6), 617-621.

Blackburn, M. W., \& Calloway, D. H. (1985). Heart rate and energy expenditure of pregnant and lactating women. The American Journal of Clinical Nutrition, 42, 1161-1169.

Botti, M., Williamson, B., Steen, K., McTaggart, J., \& Reid, E. (1998). The effect of pressure bandaging on complications and comfort in patients undergoing coronary aniography: A multicenter randomized trial. Heart Lung, 27(6), 360-373.

Bouten, C. V. C., Koekkoek, K. T. M., Verduin, M., Kodde, R., \& Janssen, J. D. (1997). A triaxial accelerometer and portable data processing unit for the assessment of daily physical activity. IEEE Transactions on Biomedical Engineering 44(3), 136-147.

Brabant, C., Bédard, S., \& Mergler, D. (1989). Cardiac strain among women workers in an industrial laundry. Ergonomics, 32(6), 615-628.

Brady, D., Raingruber, B., Peterson, J., Varnau, W., Denman, J., Resuello, R., et al. (2007). The use of knee-length versus thigh-length compression stockings and sequential compression devices. Critical Care Nursing Quarterly, 30(3), 255-262.

Buchanan, J. J., \& Horak, F. B. (2001). Transitions in a postural task: Do the recruitment and suppression of degrees of freedom stabilize posture? Experimental Brain Research, 139, 482-494.

Byrne, C., \& Lim, C. L. (2007). The ingestible telemetric body core temperature sensor: A review of validity and exercise applications. Brittish Journal of Sports Medicine, 41(3), 126-133.

Byrne, C., Lim, C. L., Chew, S. A. N., \& Ming, E. T. Y. (2005). Water versus carbohydrateelectrolyte fluid replacement during loaded marching under heat stress. Military Medicine, 170(8), 715-721.

Candas, V., Libert, J.-P., Brandenberger, G., Sagot, J.-C., \& Kahn, J.-M. (1988). Thermal and circulatory responses during prolonged exercise at different levels of hydration. Journal of Physiology, 83, 11-18.

Carter, J. E. L. (2002). The Heath-Carter Anthropometric Somatotype - Instruction Manual (pp. 1-26). San Diego, CA: San Diego State University.

Cattaneo, C. G., Frank, S. M., Hesel, T. W., El-Rahmany, H. K., Kim, L. J., \& Tran, K. M. (2000). The accuracy and precision of body temperature monitoring methods during regional and general anesthesia. Anesthesia and Analgesia, 90, 938-945.

Coris, E. E., Ramirez, A. M., \& Van Durme, D. J. (2004). Heat illness in athletes: The dangerous combination of heat, humidity, and exercise. Sports Medicine, 34(1), 9-16. 
Davis, R., Ounpuu, S., Tyburski, D., \& Gage, J. (1991). A gait analysis data collection and reduction technique. Human Movement Science, 10, 575-587.

Derave, W., Tombeux, N., Cottyn, J., Pannier, J. L., \& De Clercq, D. (2002). Treadmill exercise negatively affects visual contribution to static postural stability. International Journal of Sports Medicine, 23, 44-49.

Eagen, M., SooHoo, N., \& Cracchiolo, A., III. (2006). A cotton batting compression dressing and fiberglass cast used safely in the immediate postoperative period after hindfoot or ankle surgery. Foot and Ankle International, 27(9), 706-710.

Edwards, R. J., Belyavin, A. J., \& Harrison, M. H. (1978). Core temperature measurement in man. Aviation, Space, and Environmental Medicine, 49(11), 1289-1294.

Fanger, P. O. (1967). Calculation of thermal comfort: Introduction of a basic comfort equation. ASHRAE Transactions, 73(2), III4.1-III4.20.

Fanger, P. O. (1970). Thermal Comfort Danish Technical Press, Copenhagen.

Freyschuss, U., \& Melcher, A. (1978). Exercise energy expenditure in extreme obesity: Influence of ergonometry type and weight loss. Scandinavian Journal of Clinical and Laboratory Investigation, 38, 753-759.

Gagnon, M., Plamondon, A., Gravel, D., \& Lortie, M. (1996). Knee movement strategies differentiate expert from novice workers in asymmetrical manual materials handling. Journal of Biomechanics, 29(11), 1445-1453.

Gallagher, S. (1991). Acceptable weights and physiological cost of performing combined manual handling tasks in restricted postures. Ergonomics, 34(7), 939-952.

Garg, A., Chaffin, D. B., \& Herrin, G. D. (1978). Prediction of metabolic rates for manual materials handling jobs. American Industrial Hygiene Association Journal, 39(8), 661674.

Garg, A., \& Herrin, G. D. (1979). Stoop or squat: A biomechanical and metabolic evaluation. AlIE Transactions, 11(4), 293-302.

Garg, A., \& Saxena, U. (1979). Effects of lifting frequency and technique on physical fatigue with special reference to psychophysical methodology and metabolic rate. American Industrial Hygiene Association Journal, 40(10), 894-903.

Gilleard, W., \& Smith, T. (2007). Effect of obesity on posture and hip joint moments during a standing task, and trunk forward flexion motion. International Journal of Obesity, 31(2), 267-271.

Gisolfi, C. V. (1983). Temperature regulation during exercise: Directions - 1983. Medicine and Science in Sports and Exercise, 15(1), 15-20.

Godek, S. F., Godek, J. J., \& Bartolozzi, A. R. (2005). Hydration status in college football players during consecutive days of twice-a-day preseason practices American Journal of Sports Medicine, 33(6), 843-851.

Haagen, C. H. (1949). Synonymity, vividness, familiarity, and association value ratings of 400 pairs of common adjectives. Journal of Psychology, 27, 453-463.

Hagenouw, R. R. P. M., Bridenbaugh, P. O., van Egmond, J., \& Stuebing, R. (1986). Tourniquet pain: A volunteer study. Anesthesia and Analgesia, 65, 1175-1180.

Hattori, Y., Ono, Y., Shimaoka, M., Hiruta, S., Kamijima, M., Ichihara, G., et al. (1996). Effects of asymmetric dynamic and isometric liftings on strength/force and rating of perceived exertion. Ergonomics, 39(6), 862-876. 
Henry, S. M., Fung, J., \& Horak, F. B. (2001). Effect of stance width on multidirectional postural response. Journal of Neurophysiology, 85, 559-570.

Howe, A. S., \& Boden, B. P. (2007). Heat-related illness in athletes. American Journal of Sports Medicine, 35(8), 1384-1395.

Jackson, B. S., \& Kinney, M. R. (1978). Energy expenditure, heart rate, rhythm, and blood pressure in normal female subjects engaged in common hospitalized patient positions and modes of patient transfer. International Journal of Nursing Studies, 15, 115-128.

Jaegers, S. M. H. J., Vos, L. D. W., Rispens, P., \& Hof, A. L. (1993). The relationship between comfortable and most metabolically efficient walking speed in persons with unilateral above-knee amputation. Archives of Physical Medicine and Rehabilitation, 74, 521-525.

Jensen, R. K., Doucet, S., \& Treitz, T. (1996). Changes in segment mass and mass distribution during pregnancy. Journal of Biomechanics, 29(2), 251-256.

Jorgensen, M. J., Marras, W. S., \& Gupta, P. (2003). Cross-sectional area of the lumbar back muscles as a function of torso flexion. Clinical Biomechanics, 18, 280-286.

Jorgensen, M. J., Marras, W. S., Gupta, P., \& Waters, T. R. (2003). Effect of torso flexion on the lumbar torso extensor muscle sagittal plane moment arms. The Spine Journal, 3, 363369.

Judelson, D. A., Maresh, C. M., Farrell, M. J., Yamamoto, L. M., Armstrong, L. E., Kraemer, W. J., et al. (2007). Effect of hydration state on strength, power, and resistance exercise performance. Medicine and Science in Sports and Exercise, 39(10), 1817-1824.

Kawabata, H., Obara, K., Komiyama, H., \& Narumi, T. (2001). The effects of skirt lining properties on comfort and movement. International Journal of Consumer Studies, 25(4), 271-278.

Kielblock, A. J., Van Rensburg, J. P., \& Franz, R. M. (1986). Body cooling as a method for reducing hyperthermia: An evaluation of techniques. South African Medical Journal, 69(6), 378-380.

Knapik, J. J., Reynolds, K. L., \& Harman, E. (2004). Soldier load carriage: Historical, physiological, biomechanical, and medical aspects. Military Medicine, 169(1), 45-56.

Kraemer, W. J., Volek, J. S., Bush, J. A., Gotshalk, L. A., Wagner, P. R., Gómez, A. L., et al. (2000). Influence of compression hosiery on physiological responses to standing fatigue in women. Medicine and Science in Sports and Exercise, 32(11), 1849-1858.

Laursen, P. B., Suriano, R., Quod, M. J., Lee, H., Abbiss, C. R., Nosaka, K., et al. (2006). Core temperature and hydration status during an Ironman triathlon. British Journal of Sports Medicine, 40, 320-325.

Lavender, S. A., Li, Y. C., Andersson, G. B. J., \& Natarajan, R. N. (1999). The effects of lifting speed on the peak external forward bending, lateral bending, and twisting spine moments. Ergonomics, 42(1), 111-125.

Lenhardt, R. (2003). Monitoring and thermal management. Best Practice and Research Clinical Anaesthesiology, 17(4), 569-581.

Liu, W., Lian, Z., \& Liu, Y. (2008). Heart rate variability at different thermal comfort levels. European Journal of Applied Physiology, 103(3), 261-366.

McGibbon, C. A., \& Krebs, D. E. (2001). Age-related changes in lower trunk coordination and energy transfer during gait. Journal of Neurophysiology, 85(5), 1923-1931. 
Menze, R., McMullen, M. J., White, L. J., \& Dougherty, J. M. (1996). Core temperature monitoring of firefighters during hazardous materials training sessions. Prehospital and Disaster Medicine, 11(2), 108-111.

Meyers, L. G., Horrigan, D. J., \& Lotz, W. G. (1995). Effects of three hydration beverages on exercise performance during 60 hours of heat exposure. Aviation, Space, and Environmental Medicine, 66(11), 1052-1057.

Mitchell, D., \& Wyndham, C. H. (1969). Comparison of weighting formulas for calculating mean skin temperature Journal of Applied Physiology, 26(5), 616-622.

Mitchell, D., \& Wyndham, C. H. (1969). Comparison of weighting formulas for calculating mean skin temperature. Journal of Applied Physiology, 26(5), 616-622.

Montain, S. J., Sawka, M. N., Latzka, W. A., \& Valeri, C. R. (1998). Thermal and cardiovascular strain from hypohydration: Influence of exercise intensity. International Journal of Sports Medicine, 19(87-91), 87.

Morante, S. M., \& Brotherhood, J. R. (2007). Air temperature and physiological and subjective responses during competitive singles tennis. British Journal of Sports Medicine, 41, 773778.

Nadel, E. R., Fortney, S. M., \& Wenger, C. B. (1980). Effect of hydration state on circulatory and thermal regulations. Journal of Applied Physiology, 49(4), 715-721.

Neal, C. (1997). The assessment of knowledge and application of proper body mechanics in the workplace Orthopaedic Nursing, 16(1), 66-69.

Nevala, N., Holopainen, J., Kinnunen, O., \& Hänninen, O. (2003). Reducing the physical work load and strain of personal helpers through clothing redesign. Applied Ergonomics, 34, 557-563.

Newsom, D. M., Bolgos, G. L., Colby, L., \& Nemzek, J. A. (2004). Comparison of body surface temperature measurement and conventional methods for measuring temperature in the mouse. Contemporary Topics in Laboratory Animal Science, 43(5), 13-18.

Nicholson, A. S. (1989). A comparative study of methods for establishing load handling capabilities. Ergonomics, 32(9), 1125-1144.

Paul, J. A., Sallé, H., \& Frings-Dresen, M. H. W. (1996). Effect of posture on hip joint moment during pregnancy while performing a standing task. Clinical Biomechanics, 11(2), 111115.

Puhakka, K., Anttonen, H., Niskanen, J., \& Ryhänen, P. (1994). Calculation of mean skin temperature and changes in body heat content during paediatric anaesthesia. British Journal of Anaesthesia, 72, 548-553.

Ramakrishnan, H. K., Kadaba, M. P., \& Wootten, M. E. (1987). Lower extremity joint moments and ground reaction torque in adult gait. Biomechanics of Normal and Prosthetic Gait, 4, 87-92.

Ramakrishnan, H. K., Masiello, G., \& Kadaba, M. P. (1991). On the estimation of the three dimensional joint moments in gait. Biomechanics Symposium, 120, 333-339.

Ramakrishnan, H. K., Wootten, M. E., \& Kadaba, M. P. (1989, February 6-9). On the estimation of three dimensional joint angular motion in gait analysis. Paper presented at the 35th Annual Meeting, Orthopaedic Society, Las Vegas, Nevada.

Ramanathan, N. L. (1964). A new weighting system for mean surface temperature of the human body. Journal of Applied Physiology, 19(3), 531-533. 
Rico-Sanz, J., Frontera, W. R., Rivera, M. A., Rivera-Brown, A., Molé, P. A., \& Meredith, C. N. (1996). Effects of hyperhydration on total body water, temperature regulation, and performance of elite young soccer players in a warm climate. International Journal of Sports Medicine, 17(2), 85-91.

Roefs, J. P. A., Schrama, J. W., \& Schouten, W. G. P. (1996). Effect of temperature and food intake on metabolic rate and posture of preruminant calves Physiology and Behavior, 60(1), 203-209.

Rowland, T., Garrison, A., \& Pober, D. (2007). Determinants of endurance exercise capcity in the heat in prepubertal boys. International Journal of Sports Medicine, 28, 26-32.

Saha, D., Gard, S., Fatone, S., \& Ondra, S. (2007). The effect of trunk-flexed postures on balance and metabolic energy expenditure during standing. Spine, 32(15), 1605-1611.

Salokhe, V. M., \& Mamansari, D. U. (1995). Physical workload during selected farm operations. Journal of Human Ergology, 24(2), 161-169.

Sanchez, D., \& Grieve, D. W. (1992). The measurement and prediction of isometric lifting strength in symmetrical and asymmetrical postures. Ergonomics, 35(1), 49-64.

Sarton-Miller, I. (2006). Noninvasive assessment of energy expenditure in children. American Journal of Human Biology, 18, 600-609.

Savage, P. D., Toth, M. J., \& Ades, P. A. (2007). A re-examination of the metabolic equivalent concept in individuals with coronary heart disease. Journal of Cardiopulmonary Rehabilitation and Prevention, 27(3), 143-148.

Schenk, R. J., Doran, R. L., \& Stachura, J. J. (1996). Learning effects of a back education program. Spine, 21(19), 2183-2189.

Schiffman, J. M., Bensel, C. K., Hasselquist, L., Gregorczyk, K. N., \& Piscitelle, L. (2006). Effects of carried weight on random motion and traditional measures of postural sway. Applied Ergonomics, 37, 607-614.

Shellock, F. G., \& Rubin, S. A. (1982). Simplified and highly accurate core temperature measurements. Medical Progress through Technology, 8, 187-188.

Sookram, S. M., Barker, S., Kelly, K. D., Patton, W., Sosnowski, T., Neilson, K., et al. (2004). Can body temperature be maintained during aeromedical transport? Canadian Journal of Emergency Medicine, 4(3), 172-177.

Steptoe, A. (2000). Stress, social support, and cardiovascular activity over the working day. International Journal of Psychophysiology, 37(3), 299-308.

van Hoof, J. (2008). Forty years of Fanger's model of thermal comfort: comfort for all? Indoor Air, 18(3), 182-201.

Vezina, N., Tierney, D., \& Messing, K. (1992). When is light work heavy? Components of the physical workload of sewing machine operators working at piecework rates. Applied Ergonomics, 23(4), 268-276.

Watanuki, S., \& Murata, H. (1994). Effects of wearing compression stockings on cardiovascular responses. Annals of Physiological Anthropology, 13(3), 121-127.

Wendt, D., van Loon, L. J. C., \& van Marken Lichtenbelt, W. D. (2007). Thermoregulation during exercise in the heat. Sports Medicine, 37(8), 669-682.

Wiker, S. F., Chaffin, D. B., \& Langolf, G. D. (1989). Shoulder posture and localized muscle fatigue and discomfort. Ergonomics, 32(2), 211-237. 
Woodrow, P., May, V., Buras-Rees, S., Higgs, D., Hendrick, J., Lewis, T., et al. (2006). Comparing no-touch and tympanic thermometer temperature recordings British Journal of Nursing, 15(18), 1012-1016. 


\section{APPENDICES}

APPENDIX A. Institutional Review Board human research subjects protocol statement

Institutional Review Board

for the Protection of

Human Research Subjects

\section{Protocol Statement}

West Virginia University

Revised August 2006

Return to: IRB, PO Box 6845

Telephone: (304) 293-7073

E-Mail: irb@mail.wvu.edu 
Instructions - Guidelines Currently Under Revision (Links NOT Applicable)!

All of the protocol statement elements are explained in Chapter III of the IRB Guidelines on the Research Compliance website: http://www.wvu.edu/ rc.

For information on training and certification please see http://www.wvu.edu/ rc/irb/ethi_tra.htm.

The elements must be submitted in the order given below. All pages must be one-sided. Number all pages consecutively in pencil by section (e.g. A1 to An; B1 to Bn). All submissions must be legible and suitable for photocopying. IRB staff may return any materials that are not sufficiently legible. Do not staple or paper clip documents. Use colored paper to separate sections and multiple copies of the protocol.

For both expedited and quorum review studies, a protocol consists of:

\section{A. Protocol Statement}

The attached "Protocol Statement" constitutes the first section of a protocol. For instructions see Chapter III, Section A of the Guidelines.

\section{B. Abstract}

The abstract should include the title and a summary of the purposes and procedures. Because of the multidisciplinary nature of the Board, the abstract must be written in non-technical lay terminology.

C. Consent Forms, Assent Forms, Cover Letters, Recruitment Ads, \& HIPPA Forms Provide a copy of each consent form, assent form, cover letter(s), recruitment ad(s), and HIPAA Authorization or HIPAA Waiver form(s) intended for subjects or legal representatives. If requesting any waiver or alteration of the consent, assent or HIPAA process (see Chapter IV,

\section{Discussion}

The discussion section must give complete information (in the boxes provided) concerning each of the items described in Chapter III, Section D of the Guidelines. If any item does not apply to the proposed activity, type N/A in the box provided.

\section{E. Attachments}

See Chapter III, Section E of the Guidelines for a full description of attachments to a protocol. These include telephone texts; a copy of surveys or other test instruments that will be used; letter(s) granting permission to use facilities or resources from other institution(s) or organization(s) on their letterhead; consultant agreements; approval letters from radiation safety and biohazards committees; or any external protocol.

\section{Submission Procedures and Dates - Do not staple or paper clip documents; use colored paper to separate sections and multiple copies of the protocol.}

\section{Expedited Review}

In order to qualify for expedited review, the protocol must fit exactly in one of categories 1-6 AND be indicated on the Protocol Statement (see Chapter II, Section B of the Guidelines). If the research qualifies for expedited review, submit the original protocol to the IRB office. There are no submission deadlines for this category.

\section{Quorum Review}

Protocols requiring quorum review (see Chapter II, Section C of the Guidelines) will be reviewed at regularly scheduled IRB meetings (which occur on the 2nd and 4th Wednesdays of each month - Meeting Dates and Submission Deadlines). In order for a protocol to be considered, submit the original plus 21 copies for all new submissions (includes protocol statement, abstract, consent and assent forms [if applicable], discussion, and attachments) and the original plus 2 copies of 
all material provided by a sponsor (this could include a sponsor protocol, addendum material, investigator brochure, IND safety reports, etc.). All material must reach the compliance office by the deadline date.

For amendments requiring quorum review (see Chapter V, Section A of the Guidelines), submit the original plus 21 copies of the amendment and attachments (original plus 2 of the sponsor materials [when applicable]). 


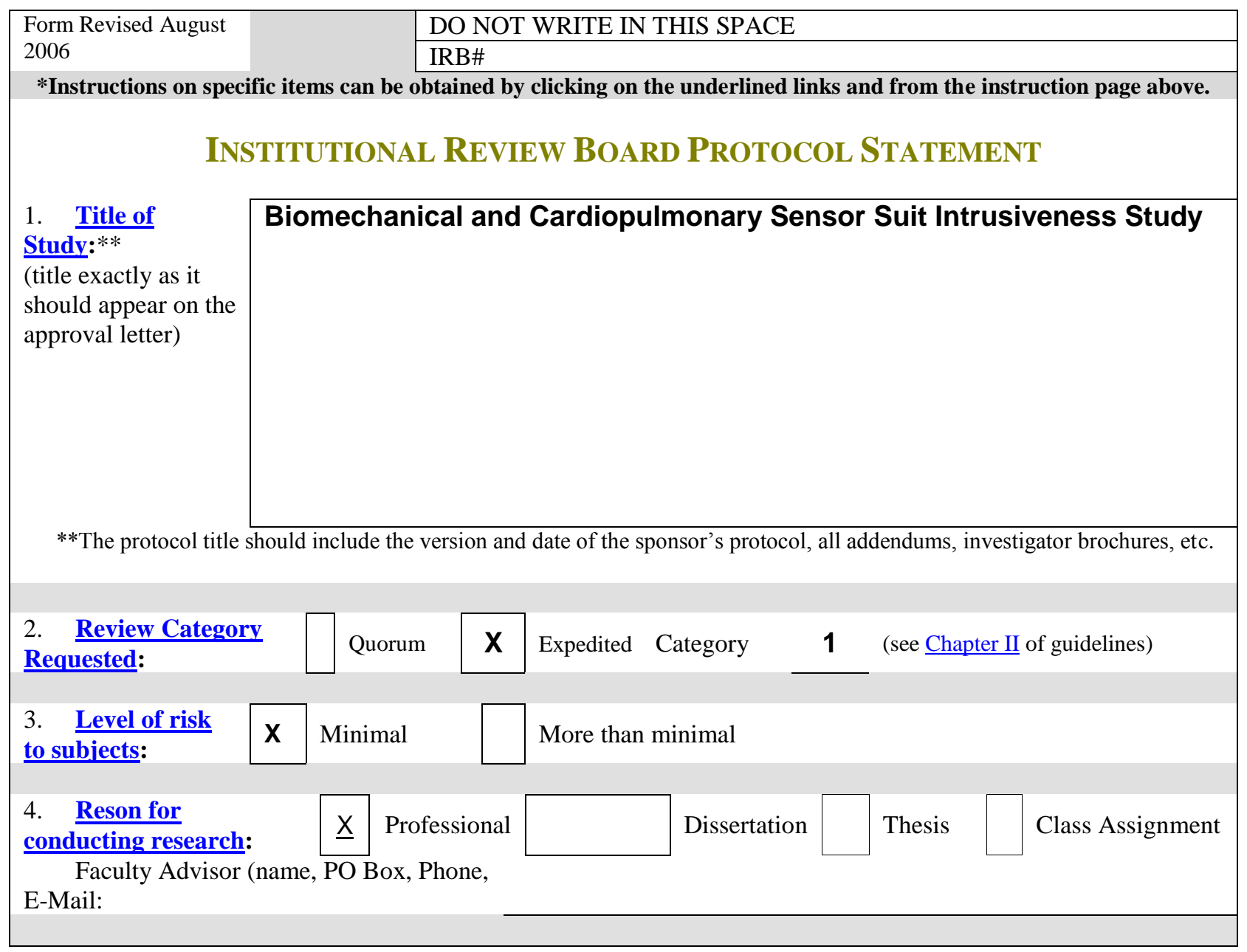

\begin{tabular}{|l|l|l|l|l|l|}
\hline \multicolumn{7}{|l|}{ 5. Investigators: (list all key personnel, principal investigator first; attach additional sheets if necessary) } \\
\hline $\begin{array}{l}\text { Name (type or print FULL } \\
\text { name) }\end{array}$ & Signature & $\begin{array}{l}\text { College/School \& } \\
\text { Department }\end{array}$ & PO Box & E-Mail \\
\hline PI & $\begin{array}{l}\text { Steven F. Wiker, } \\
\text { Ph.D. }\end{array}$ & & CEMR & 6070 & sfwiker@mail.wvu.edu \\
\hline Co-I & Mark Jackson & & CEMR & 6070 & mjackson@cdc.gov \\
\hline Co-I & Anu Maduri & & CEMR & 6070 & amaduri@cdc.gov \\
\hline Co-I & Brad Westfall & & CEMR & 6070 & bwestfall@cdc.gov \\
\hline Co-I & Amie King & & CEMR & 6070 & aking@mix.wvu.edu \\
\hline Co-I & & & & 6070 & jmcferron@gmail.com \\
\hline Other & Joe McFerron & & CEMR & 6 & \\
\hline
\end{tabular}




\begin{tabular}{|l|l|l|l|l|l|}
\hline Other & Erik Sinsel & & CEMR & 6070 & eriksinsel@excite.com \\
\hline & Name/Initials & E-Mail & Phone & Fax & \\
\hline PI & $\begin{array}{l}\text { Steven F. } \\
\text { Wiker }\end{array}$ & sfwiker@mail.wvu & $293-4607 \times 3733$ & $\begin{array}{l}293- \\
4907\end{array}$ & \\
\hline
\end{tabular}

6. Contact Person: (if different from principal investigator)

\begin{tabular}{|l|l|l|l|l|l|l|}
\hline Name & \multicolumn{3}{|l|}{$\begin{array}{l}\text { College/ } \\
\text { School }\end{array}$} & Dept. & \\
\hline PO Box & Phone & Fax & E-Mail & \\
\hline
\end{tabular}

7. Drug/Device Study Details:

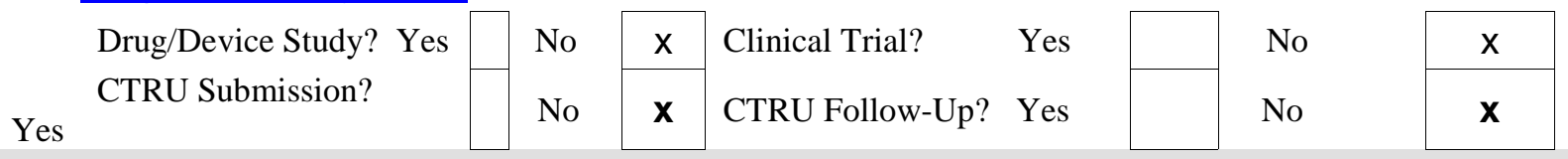

8. Will Investigational Drugs or Devices Be Used? Yes

$$
\begin{array}{ll}
\text { If Yes: } & \text { IND\# } \\
& \text { IDE\# }
\end{array}
$$

Manufacturer:

Manufacturer:

Will a Humanitarian Device Exemption (HDE) Be Used? Yes

If Yes: HDE\#

Manufacturer: No $x \quad \begin{aligned} & \text { See WVU Hospital Pharmacy } \\ & \text { Policy for drug use. }\end{aligned}$

9. Source of Funding Support:

Is funding applied for?

Yes

$x$ No $\square$

Federal Name: National Institutes for Occupational Safety and Health

State Name:

Private Name:

Yes

Has the IRB Fee been paid:

No

See IRB FEE Memo for Industry

Sponsored Protocols.

Internal Name:

10. Human Subject Details: Total number of subjects to be enrolled

nationwide

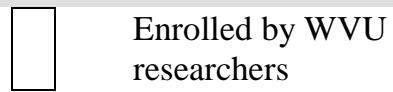

researchers

Setting for interaction with subjects (provide name of institution, address, and brief description):

Research will be conducted within the Ergonomics Laboratory, 109 ERB, West Virginia University. 


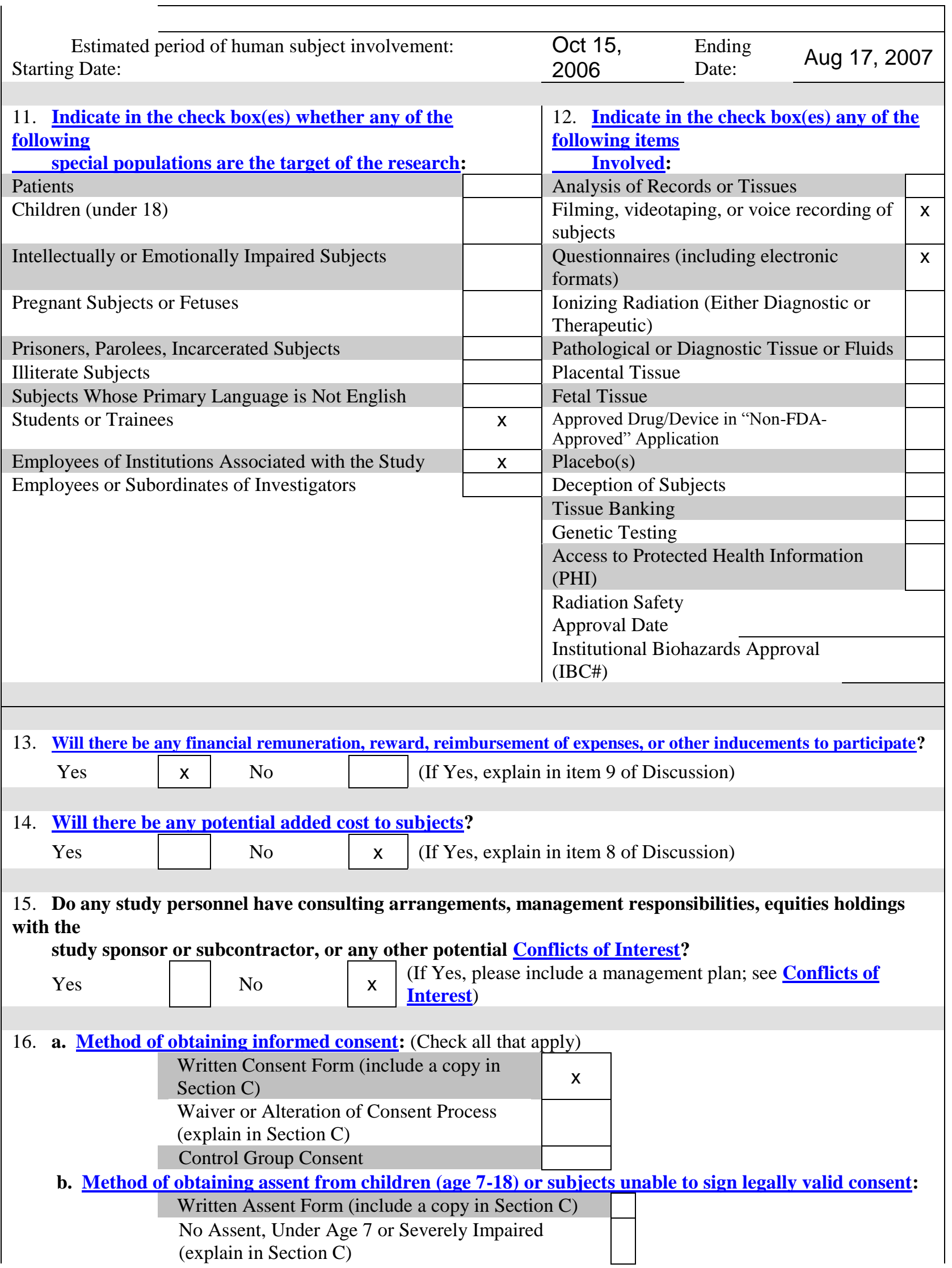




\section{Waiver or Alteration of Assent Process (explain in \\ Section C) \\ Control Group Assent}

17. Method of obtaining HIPAA Authorization: (See http://www.wvu.edu/ rc/irb/irb_guid/append_g.htm)

Individual Authorization

Waiver of Individual

Authorization

18. Will you use a cover letter?

Yes

No

$\mathrm{x}$ (If Yes, include a copy in Section C)

19. Will you use a recruitment ad?

Yes

$x$ No

(If Yes, include a copy in Section C)

20. Signatures: (The Board will not review the protocol without the signature of the departmental chair, dean and if appropriate, hospital administration, faculty advisor, or others. No sign off from the dean of the School of Medicine is necessary due to interdepartmental policies. By signing, department chairs acknowledge approval of this study on the basis of scientific merit and compliance with applicable professional standards; deans and other administrators signify their approval of the use of resources and faculty and student effort on the study. Multi-Unit protocols require the signatures of each chair and dean)

\begin{tabular}{|l|l|l|l|l|}
\hline & Name (Type or Print) & Title & Signature & Date \\
\hline Dean & Eugene Cilento & Dean, CEMR & & \\
\hline Dean & & & & \\
\hline Dept. Chair & Wafik Iskander & Chair, IMSE & & \\
\hline Dept. Chair & & & & \\
\hline Hospital Admin. & & & & \\
\hline Faculty Advisor & Steven Wiker & PI and Lab Director & & \\
\hline Other & & & & \\
\hline
\end{tabular}




\section{B. ABSTRACT}

The Ergonomics Laboratory and the National Institutes of Occupational Safety and Health (NIOSH) are developing a sensor suit that will measure biomechanical and physiological stresses that are experienced by workers when they perform their jobs. This information has been difficult to measure in the past. If the sensor suit can be developed, workers will be able to wear it while working. The suit will record important information needed to assess whether jobs are safe or not. The suit will provide information needed by engineers for development of better design of jobs to improve human performance and to reduce risk of developing muscle, bone, heart and lung injuries and illnesses in the workplace. This study is designed to measure the intrusiveness of the sensor suit. Pilot tests showed that current sensor suits developed by the ABACAS team can produce material changes in kinematics and kinetics, and reported tolerance levels (e.g., cross-modal matching of reports of fatigue and discomfort). Wearers may have altered movement patterns when possible to: a) control metabolic heat production in response to ensembles that added Clo (clothing insulation unit) factors, b) reduce energy expenditure rates to extend endurance, c) to instrumentation induced changes in joint stiffness, d) changes in centers of mass with added instrumentation mass, or e) reduce mechanical irritation or chaffing of skin by ensembles. If intrusions are a result of one or more of these factors, we need to understand the bases early on. This information will feed into design recommendations and selection criteria for various combinations of ensembles, and used to prevent intrusion in the design of future suits. Subjects will wear all 4 prototype sensor suits and one sham suit that allows us to achieve a balanced exposure to design features across suits. Subjects will perform four standard industrial tasks (lift, carry, lower, walk) in a cyclic manner while a computerized video analysis of posture, is performed along with recording body core and skin temperatures, heart rate, and we collect self-reports of fatigue and discomfort are made. The subject will continue to perform the simulated industrial work tasks for up to 4 hours if no stopping criteria are met. Subjects will be given a few days to rest between the next replication of the experimental trial in which they wear another prototype suit. Each subject will undergo five test days/periods. Data obtained will be analyzed to determine what sensor configuration characteristics avoid posture intrusion and alteration of physiological responses.

\section{CONSENT FORMS, ASSENT FORMS, COVER LETTERS, HIPAA FORMS, RECRUITMENT ADS}

See Attached.

\section{Discussion}

Provide complete information concerning each of the following items, numbered as indicated. If any item does not apply to the proposed activity, type NA (Not Applicable) by that item number. Number all pages consecutively D1-Dn. Pages may be numbered by hand.

\section{1. "Purposes"}

The National Institutes of Occupational Safety and Health (NIOSH) has recognized the need to develop and to deploy technology that allows accurate, reliable, feasible and sustained measurement of biomechanical and physiological stress/strain exposures in structured and unstructured work environs. The Advanced Biomechanical and Cardiopulmonary Assessment Suit (ABACAS) project aims to develop, validate, deploy and use a wearable instrumentation suite that permits measurement, recording and assessment of environmental and job stressors and resultant human physiological, biomechanical and psychometric response in laboratory and workplace environments.

Pilot tests showed that current sensor suits developed by the ABACAS team can produce material changes in kinematics and kinetics, and reported tolerance levels (e.g., cross-modal matching of reports of fatigue and discomfort). Wearers may have altered movement patterns when possible to: a) control metabolic heat production in response to ensembles that added Clo factors, b) reduce energy expenditure rates to extend endurance, c) to instrumentation induced changes in joint stiffness, d) changes in centers of mass with added instrumentation mass, or e) 
reduce mechanical irritation of skin by ensembles. If intrusions are a result of one or more of these factors, we need to understand the bases early on. This information will feed into design recommendations and selection criteria for various combinations of ensembles, and used to prevent intrusion in the design of future suits.

Results from this study will help us to design sensor suits that are comfortable and that can be worn without affecting postures and cardiopulmonary burdens when working in industrial environments. 
Subjects will be asked to don five sensor-based prototype suits that possess different designs. One sensor configuration will serve as a control condition in which subjects will wear a set of light-weight light-reflective markers (referred to as the Qualysis configuration). With the control sensor suite, we will have subjects wear standard shorts and sleeveless shirts made of lightweight cotton.

Every sensor suits configuration will require subjects wear the a arm band heart rate monitor, skin temperature sensor, and swallow a disposable telemetry pill for transmitting core or gut temperature to a receiver in the lab. The other ensembles worn will be the commerciallyavailable Measurerand $^{\mathrm{TM}}$ Tape Posture Recording System, the commercially-available K4B2 ${ }^{\mathrm{TM}}$ cardio-pulmonary recording system, the commercially-available Lifevest ${ }^{\mathrm{TM}}$ cardio-pulmonary recording system, and a "sham" sensor suit that will distribute cloth and weight about the body to permit use to have a balanced exposure to variations in ensemble Clo value, center of mass locus, mass distribution on the torso and arms, and presence and absence of bands used to hold sensors in place.

Pretesting Subjects:

Anthropometric measurements will be made (e.g., body mass, stature, link lengths) to enable us to design their lifting and carriage tasks to not exceed 30 percent of their estimated exertion capacity, to insure that the National Institutes of Occupational Safety and Health recommended safe load lifting and aerobic power demands are not exceeded (e.g., $3.5 \mathrm{Kcal} / \mathrm{min}$ and 5.0 $\mathrm{Kcal} / \mathrm{min}$ for women and men, respectively). The computations will be made and all tasks will be specified for that subject. The subject will leave after the initial informed consent briefing and preliminary acceptance testing. They will return at scheduled time to perform the tasks while wearing a randomly ordered sensor suit configuration.

All workloads experienced will be adjusted to meet subject capacities. Prior to conduct of the first trial, subjects will observe a videotape presentation of a demonstration of the task performance. They will be introduced to every ensemble that they will be asked to wear (they will see one of the team members wearing each of the ensembles in the videotape), and they can ask questions about any of the apparatus or procedures. We will then ask the subject to perform a series of progressively loaded box lifts and carries to estimate their strength capacity. A series of three or four progressively heavier boxes will be lifted and lowered and then carried to determine their estimate of the percent of their strength capacity that is estimated by subjects. Subjects will rate the level of difficult from 0 (No Effort) to 10 (Maximum Effort). No test exertions will exceed a rating of 5 or 50 percent of their tolerance level. The exertion levels will fall below recommended physical activity limits that are used by NIOSH to avoid onset of systemic fatigue in the American workforce.

Testing:

Instrumentation ensembles will be classified or scored dimensionally using: magnitude of Clo (unit of clothing insulation) and Clo distribution, total mass added to the body and its distribution, mounting strategies (e.g., elastic banding, etc.), and impact upon joint stiffness as shown in Table 1. Introducing dummy ensembles that provide sufficient orthogonalization to permit feature main and interaction effect analysis to determine which combinations of feature degrade wearer tolerance and endurance.

Table 1. Design feature states for ABACAS prototype ensembles that will be tested.

\begin{tabular}{|l|l|l|l|l|l|}
\hline \multirow{2}{*}{ Design Feature } & \multicolumn{5}{|c|}{ Ensemble } \\
\cline { 2 - 6 } & $\begin{array}{c}\text { Qualisys \& } \\
\text { HR Monitor }\end{array}$ & $\begin{array}{c}\text { Measurand } \\
\text { Tape }\end{array}$ & K4B $^{2}$ & LifeShirt & Sham \\
\hline
\end{tabular}




\begin{tabular}{|l|l|c|c|c|c|c|}
\hline Banding & - & + & - & - & + \\
\cline { 2 - 6 } Clo & - & + & - & + & - \\
\hline COM & - & + & 0 & 0 & - \\
\hline $\begin{array}{l}\text { Clothing Tension } \\
\text { About Joints }\end{array}$ & - & + & - & - & + \\
\hline Mass & - & + & 0 & 0 & 0 \\
\hline Clo Region & & Torso & & Torso & \\
\hline Mass Region & & Torso & Waist & Torso & Arms \\
\hline
\end{tabular}

Using the ensembles noted in Table 1, wearers will perform a representative ensemble of industrial manual materials handling tasks. The tasks will differ in energy expenditure demands and ranges of motion, while the dependent kinematic and physiological metrics, shown in Figure 1 , are continuously sampled at a minimum of $60 \mathrm{~Hz}$ or greater. Borg effort and discomfort ratings, obtained every 30 minutes following the method of Wiker et al. (1989), will serve as dependent metrics along with posture (joint angles) and heart rate and/or oxygen consumption.

\begin{tabular}{|c|c|}
\hline Dependent Metrics & Ensemble Factor \\
\hline $\begin{array}{l}\text { Joint Angles } \\
\text { Heart Rate/Oxygen Consumption } \\
\text { Borg Scale Rating } \\
\text { Global Discomfort } \\
\text { Regional Discomfort }\end{array}$ & $=\mid \begin{array}{l}\text { Added Mass } \\
\text { CLO Magnitude } \\
\text { Cross-Joint Resistance } \\
\text { COM Change } \\
\text { Constriction Bands }\end{array}$ \\
\hline
\end{tabular}

\begin{tabular}{|l|l|}
\hline Task Factor \\
\hline \hline Duration \\
Kcal/Min \\
Ranges of Motion \\
Exertion Frequency
\end{tabular}$+$\begin{tabular}{l}
\hline Subject Fact \\
\hline \hline Age \\
Gender \\
\hline
\end{tabular}

Figure 1. General paradigm for developing descriptive multiple regression models to determine the basis and magnitude intrusion response to ABACAS ensemble design features.

Five ensembles will be tested in four cyclic tasks in which range of motion and energy expenditure demands differ materially but fall within design safe design guidelines based upon NIOSH WPG and energy expenditure models. Each ensemble will be tested on one day, with at least two days of rest between test sessions.

During test sessions, subjects will continue the cycles of lift, carry, lower and walk with periodic seated rest of 5 minutes for every 15 minutes of task performance. The activity will continue until subjects reach one of the trial stopping criteria (e.g., report of exertion or discomfort at 6 out of 10 , physiological metrics exceed approved limits (e.g., HR $>40$ percent of cardiac reserve ((220-Age)-resting HR), core temperatures exceeding 39 deg $\mathrm{C}$, or task duration reaches 2 hours). Each subject will be randomly assigned an ensemble and task to perform and will wear each ensemble once.

All subjects will be tested across 5 days to be scheduled across a one-month period. Each experimental trial session will take at least $1 \mathrm{hr}$ but no more than four hours to complete.

3. "Radiation"

No exposure to radiation.

4. "Infectious Agents or Biohazards"

No exposure to infectious agents or biohazards. 


\section{5. "Deception and Debriefing"}

No deception is involved.

6. "Subjects"

Approximately 30 currently working males and females of working age (18 to 55 years of age) who report that they are healthy and have no current history of back or joint injury or disease will be invited to participate in this study. Subjects will be recruited by advertisements that will be posted at West Virginia University and about the local community.

7. "Costs of Participation"

We will collect data during periods that do not require that subjects miss work. There are no other costs associated with participation.

8. "Payments to Subjects"

Subjects will be paid $\$ 10$ for an initial informed consent briefing and anthropometry measurement session of 1 hour. Thereafter, they will receive $\$ 40$ for each test session and $\$ 210$ for the entire study if they participate in all five test sessions and the initial hour briefing and anthropometry measurement session.

9. "Benefits"

This research will benefit society, but will not provide any immediate benefit to the subject.

10. "Risks and Discomforts"

Risk of musculoskeletal injury or discomfort is no greater than that experienced when performing well-designed and safe industrial work tasks. Some subjects, who do not perform physical work or who do not exercise regularly, may experience some detectable muscular sensations or either tightness or soreness a day or two after completing the trial.

11. "Alternatives to Participation"

There are no alternative forms of participation.

12. "Intervention (Researcher Response to Unanticipated Adverse Events)"

Dr. Wiker will report any unanticipated adverse events. There is no intervention protocol beyond referring the subject to their personal physician.

13. "Questionnaires or Surveys with 'Sensitive Questions"'

The subject survey or participation questionnaire asks subjects to report whether or not they have experienced a significant low-back injury or injury to any joint or bone, or have received a diagnosis of any bone or joint disease. Prospective subjects will be informed that they will complete a health screening questionnaire to determine if they have any of the aforementioned medical histories, and that if they do, they will not be allowed to participate in the study. This usually allows candidate subjects to withdraw before reporting to the lab and completing any questions that they may find sensitive. 


\section{4. "Confidentiality"}

All data recordings will be maintained in our computer network that is password protected and resides in a constantly locked Ergonomics Laboratory. All subjects will be assigned codenames for identification of their datasets. Digital video records are captured and stored until we can confirm that the computerized analysis of subject postures is correct. Once data integrity checks are completed, the video record is destroyed. At that point, the data cannot be linked to any particular subject, nor will anyone be able to link an individual to this study.

\section{5. "Principal Investigator"}

Dr. Wiker has conducted invasive and noninvasive human physiological and biomechanical studies like that proposed for over 20 years without any subject injury or adverse effects.

Dr. Wiker conducts basic and applied research, consults and teaches in the fields of ergonomics/human factors, occupational biomechanics, work/stress physiology and safety engineering. The objectives of the research are to improve worker health, safety, and performance by improving working environment, equipment, and job design. His efforts have focused upon the identification and control of performance, safety and health problems that arise from biomechanical, physiological, or perceptual-cognition-motor problems that are encountered in the industrial workplace, aboard aerospace and marine vehicles, or when using products. Efforts are often incorporated into: a) design guidelines, specifications or standards, b) computer-models for hazard identification and implementation of administrative or engineering controls of biomechanical, physiological, and perceptual-motor related performance, health, and safety problems, or c) improved design and usability of equipment or products.

Dr. Wiker's education and degrees are provided below:

\begin{tabular}{|l|l|l|}
\hline Year & Degree & Institution \\
\hline 1986 & $\begin{array}{l}\text { Ph.D. Industrial \& Operations } \\
\text { Engineering }\end{array}$ & University of Michigan, Ann Arbor \\
\hline 1982 & $\begin{array}{l}\text { M.S. Industrial \& Operations } \\
\text { Engineering }\end{array}$ & University of Michigan, Ann Arbor \\
\hline 1981 & M.S. Biological Sciences (physiology) & George Washington University \\
\hline 1975 & B.S. Physiology & University of California, Davis \\
\hline
\end{tabular}

Representative Recent Publications:

1. Schwerha, D. J., Wiker, S.F. and Jaraiedi, M. Aging and distraction resistance during psychomotor task learning. Submitted.

2. Zhou, W., Duffie, N.A. and Wiker, S.F. Impact of grasp posture, force and fatigue upon human-controlled force-reflective master-controller model dynamics. Submitted.

3. McDowell, T. W., Wiker, S. F., Dong, R. G. and Welcome, D. E. Effects of vibration on grip and push force-recall accuracy. Submitted.

4. McDowell, T. W., Wiker, S. F., Dong, R. G., Welcome, D. E. and Schopper, A. W. (2006) Evaluation of psychometric estimates of vibratory hand-tool grip and push forces. Proceedings of the First American Conference on Human Vibration. Morgantown, WV, 10-12 June.

5. Wiker, S. F., Schwerha, D. J. and Jaraiedi, M. (2006) Impact of auditory and visual distractors upon manual assembly task: learning among older workers with different levels of spatial reasoning and field dependence. Proceedings of the $50^{\text {th }}$ Annual Meeting of the Human Factors and Ergonomics Society. San Francisco, California, October 16-20. 
6. Schwerha, D. J., Wiker, S. F. and Jaraiedi, M. (2006) Impact of age and distractors upon learning a manual assembly task. Proceedings of the International Ergonomics Association. 10-15 July, Maastricht, Netherlands.

7. McDowell, T. W., Wiker, S. F., Dong, R. G., Welcome, D. E. and Schopper, A. W. (2006) Evaluation of psychometric estimates of vibratory hand-tool grip and push forces. Intl $\mathbf{J}$ Industrial Ergonomics 36(2):119-128.

8. Wiker, S. F. (2005) Ergonomic risks, interventions and economic gains. Proceedings of the Eighth Conference and Exhibition on Occupational Safety and Health. 28-29 June, Kuala Lumpur, Malaysia.

9. Wiker, S. F. (2005) Impact of design features upon perceived tool usability and safety. Proceedings of International Society for Optical Engineering: Intelligent Systems in Design and Manufacturing VI 5999(OE05-SA108-29):R1-R14.

10. Wiker, S. F. (2005) Challenges facing developers of CAD/CAM models that seek to predict human working postures. Proceedings of International Society for Optical Engineering: Intelligent Systems in Design and Manufacturing VI 5999(OE05-SA108-28):Q1-Q7.

11. Wiker, S. F. (2003) Statistical challenges facing development of epidemiologically-validated low-back injury risk predictive and descriptive models using biomechanical, anthropometric or consensus-based ergonomic risk prevention guidelines. Seminars in Spine Surgery 15(1): 3-15.

12. Myers, A., Baker, S. P., Li, G., Smith, G., Wiker, S.F., Liang, K. and Johnson, J. (1999) Back injury in municipal workers: A case-control study. Am J Public Health 89(7): 1036-1041.

\section{6. "Other Key Personnel"}

Joe McFerron holds a BSE in Bioengineering from University of Pittsburgh (2005), is a graduate student studying under Dr. Wiker. Erik Sinsel is a doctoral student studying under Dr. Wiker and holds a Masters degree (WVU, 2006) in computer science with focus on neural network development. Both students have taken biomechanics, physiological and ergonomics graduate coursework taught by Dr. Wiker. Both of these students have built the experimental apparatus, have programmed trial control protocols, and are intimately familiar with the instrumentation involved in the study. LCDR Mark Jackson, USPHS, Anu Maduri, and Brad Westfall from $\mathrm{NIOSH}$, Morgantown have appointments as Research Associates in the Department of Industrial and Management Engineering, College of Engineering and Mineral Resources, West Virginia University. Each of the NIOSH personnel have undergone Human Subjects Training and have experience in conducting this study protocol.

17. "Consultants, Collaborators, and Non-WVU Personnel"

$\mathrm{NIOSH}$ investigators will be assisting in the experiments; each as an appointment as a Research Associate within the College of Engineering and Mineral Resources.

\section{E. ATTACHMENTS}

Page Topic

E1. Subject data and screening questionnaire.

E2. Subject Recruiting Advertisement

E3. Borg Exertion Rating and Regional Discomfort Questionnaire.

E4. Stretching exercises

E5. Memorandum of support 
APPENDIX B. Subject screening questionnaire

\section{ABACAS Subject Screening Questionnaire}

\section{Instructions}

Please answer the following questions about your health status and physical activity.

We will use this information to determine if your health status or level of daily physical activity match requirements for participation in this study.

All information provided is confidential and cannot be linked to you personally.

\section{Subject Anthropometry and Demographic Information}

You have been given a random code so that any information provided cannot be linked to you. Please complete the following questions.

\section{Your Code Number is:}

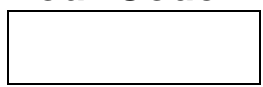

2. Gender:

O Male

O Female

3. Your age (years):

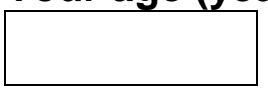

4. Highest degree of education:

$O$ Less than high school

$\bigcirc$ High School Diploma

O Some College

O College Graduate

$\bigcirc$ Graduate/Professional Degree

5. Your standing height in inches:

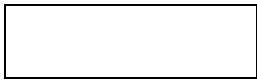

6. Your body weight in pounds: 


\section{Screening Health History}

7. Have you ever been diagnosed with a heart condition?

O Yes

O No

8. Do you ever feel pain in your chest during physical activity?

O Yes

O No

9. Have you experienced chest pains when not doing physical activity?

O Yes

O No

10. Have you sensed periods of skipped or irregular heart beats?

O Yes

O No

11. Do you experience dizziness or fainting?

$\mathrm{O}$ Yes

O No

12. Have you ever been told you have high blood pressure or are you taking medication for blood pressure or any other heart condition?

O Yes

O No

13. Do you have any existing bone or joint problem that could be made worse by physical activity?

O Yes

O No

14. Do you experience shortness of breath during only mild exertion?

O Yes

O No 
15. Do you have either Asthma or Diabetes Mellitus?

O Yes

O No

16. Are you currently taking any prescribed medication that has an impact on your physical activity?

$\mathrm{O}$ Yes

O No

Drug:

17. Are you pregnant or have you given birth in the last 6 weeks?

O Yes

O No

18. Have you recently undergone surgery or are you carrying any injury?

O Yes

O No

19. Have you been diagnosed or told that you have high blood pressure?

O Yes

O No

20. Have you been diagnosed or told that you have a bone or joint disease?

O Yes

O No

\section{Current Exercise and Work History}

Please use the following questions to describe your weekly work or exercise activities.

21. How often do you exercise during the week?

O None

$O$ Less than 30 minutes per week

O Between 31 and 90 minutes a week

O More than 90 minutes per week 


\section{Are you retired?}

O No

$\bigcirc$ Yes (Skip to question 26)

\section{What is your current occupation?}

\section{How many hours a week do you work?}

Less than 20 hours per week

O 21 to 40 hours per week

More than 40 hours per week

\section{Please rate the level of physical work activity that is required in your job:}

$O$ Office work or seated activity for the majority of the day

A combination of seated and standing work without significant physical work

O Standing for the majority of day but no significant physical work

Perform physical work that increases your rate of breathing or sweating from time to time

Perform physical work in which you breath heavy from time to time, sweat, or choose to take short rest breaks

$\bigcirc$ Perform physical work that requires greater than typical strength, physical effort, and endurance

\section{Submitting Questionnaire}

This is a general screening questionnaire. We are conducting a series of experiments which involve different levels of subject participation. Please let us know the level of participation that would be acceptable to you. We will match you to a study that will fit into your free time.

Please note that most experiments will involve a 3-4 hour test session. If multiple sessions are required, they will be scheduled throughout a couple of week or monthlong periods. Test sessions will be scheduled with you to make participation convenient and to provide sufficient separation between study sessions. 
26. If you are able to participate in this study, at what level of participation is acceptable to you?

O None

Part of one day

O One day

3-4 hours per session for 3 to 5 days schedules throughout a month

$\bigcirc$ More time if test sessions are schedules to avoid conflicts with my personal schedule

27. Preferred times to participate in this study (you can give more than one answer):

$\square$ Weekday mornings

Weekday afternoons

$\square$ Weekday evenings

$\square$ Saturday mornings

$\square$ Saturday afternoons

$\square$ Saturday evenings

\section{Any scheduling considerations you want us to consider?}

- END - 
APPENDIX C. Discomfort, fatigue, and temperature questionnaire

$\mid$
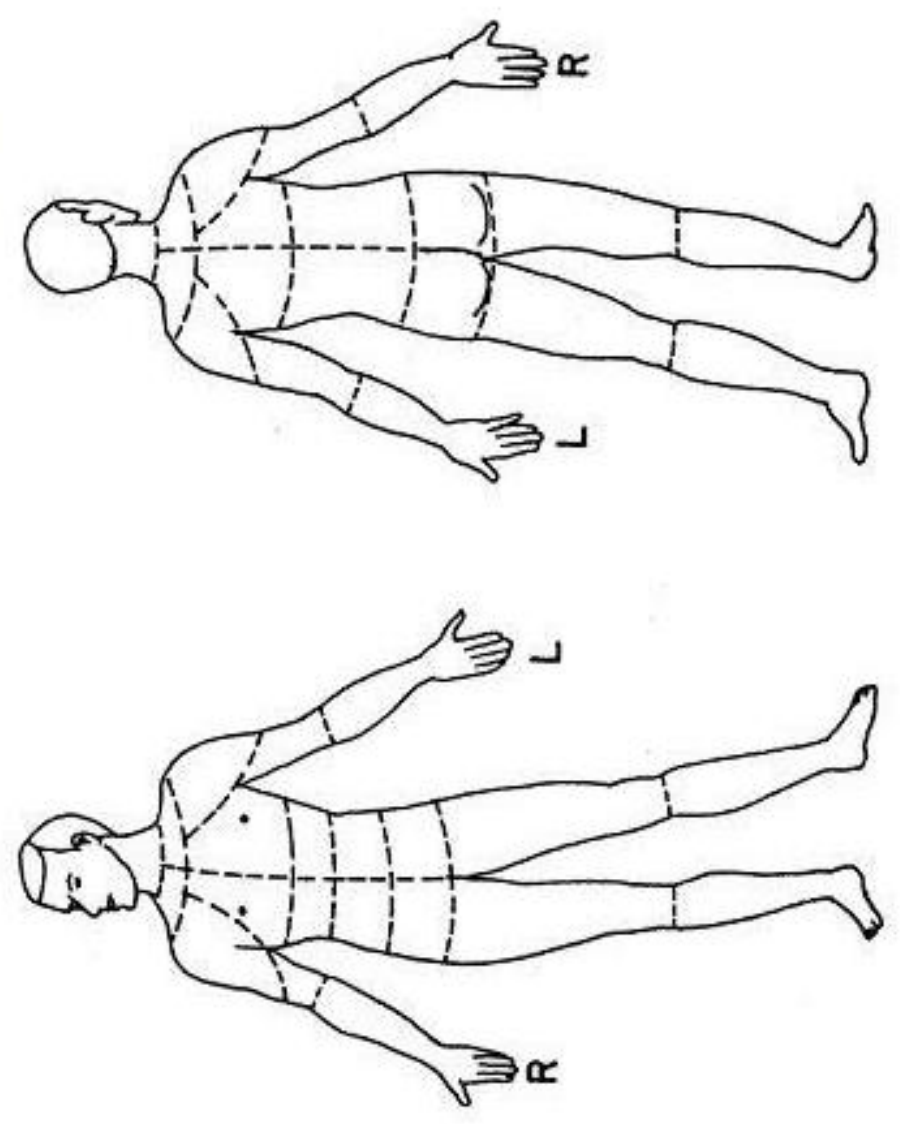

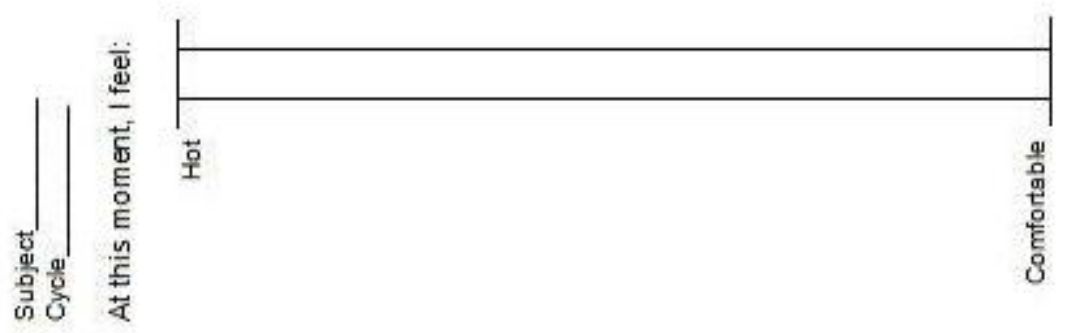

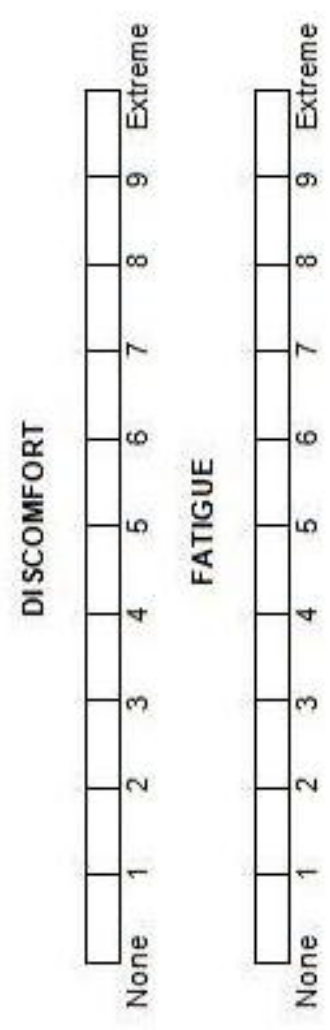




\section{Plug-in-Gait Marker Placement}

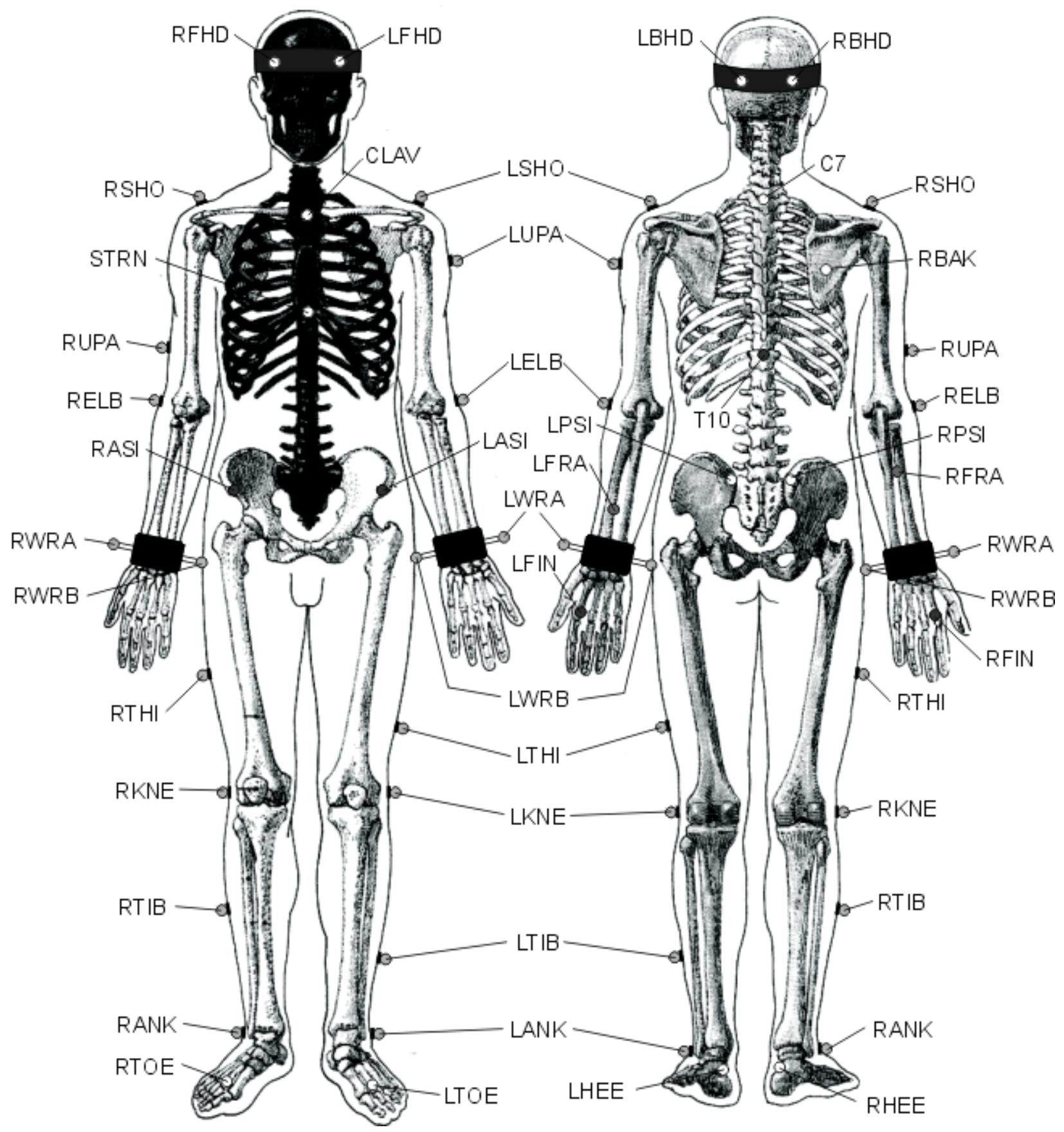

The following describes in detail where the Plug-in-Gait markers should be placed on the subject. Where left side markers only are listed, the positioning is identical for the right side. 


\section{Upper Body}

Head Markers

\begin{tabular}{|l|l|l|}
\hline LFHD & $\begin{array}{l}\text { Left front } \\
\text { head }\end{array}$ & Located approximately over the left temple \\
\hline RFHD & $\begin{array}{l}\text { Right front } \\
\text { head }\end{array}$ & Located approximately over the right temple \\
\hline LBHD & $\begin{array}{l}\text { Left back } \\
\text { head }\end{array}$ & $\begin{array}{l}\text { Placed on the back of the head, roughly in a horizontal plane of the } \\
\text { front head markers }\end{array}$ \\
\hline RBHD & $\begin{array}{l}\text { Right back } \\
\text { head }\end{array}$ & $\begin{array}{l}\text { Placed on the back of the head, roughly in a horizontal plane of the } \\
\text { front head markers }\end{array}$ \\
\hline
\end{tabular}

The markers over the temples define the origin, and the scale of the head. The rear markers define its orientation. If they cannot be placed level with the front markers, and the head is level in the static trial, tick the "Head Level" check box under options on "Run static model" in the pipeline when processing the static trial. Many users buy a headband and permanently attach markers to it.

\section{Torso Markers}

\begin{tabular}{|l|l|l|}
\hline C7 & $\begin{array}{l}7^{\text {th }} \text { Cervical } \\
\text { Vertebrae }\end{array}$ & Spinous process of the 7th cervical vertebrae \\
\hline T10 & $\begin{array}{l}10^{\text {th }} \text { Thoracic } \\
\text { Vertebrae }\end{array}$ & Spinous Process of the 10th thoracic vertebrae \\
\hline CLAV & Clavicle & Jugular Notch where the clavicles meet the sternum \\
\hline STRN & Sternum & Xiphoid process of the Sternum \\
\hline RBAK & Right Back & $\begin{array}{l}\text { Placed in the middle of the right scapula. This marker has no } \\
\text { symmetrical marker on the left side. This asymmetry helps the auto- } \\
\text { labeling routine determine right from left on the subject. }\end{array}$ \\
\hline
\end{tabular}

C7, T10, CLAV, STRN define a plane hence their lateral positioning is most important.

\section{Arm Markers}

\begin{tabular}{|l|l|l|}
\hline LSHO & $\begin{array}{l}\text { Left shoulder } \\
\text { marker }\end{array}$ & Placed on the Acromio-clavicular joint \\
\hline LUPA & $\begin{array}{l}\text { Left upper } \\
\text { arm marker }\end{array}$ & $\begin{array}{l}\text { Placed on the upper arm between the elbow and shoulder markers. } \\
\text { Should be placed asymmetrically with RUPA }\end{array}$ \\
\hline LELB & Left elbow & Placed on lateral epicondyle approximating elbow joint axis \\
\hline LFRA & $\begin{array}{l}\text { Left forearm } \\
\text { marker }\end{array}$ & $\begin{array}{l}\text { Placed on the lower arm between the wrist and elbow markers. Should } \\
\text { be placed asymmetrically with RFRA }\end{array}$ \\
\hline LWRA & $\begin{array}{l}\text { Left wrist } \\
\text { marker A }\end{array}$ & Left wrist bar thumb side \\
\hline LWRB & $\begin{array}{l}\text { Left wrist } \\
\text { marker B }\end{array}$ & Left wrist bar pinkie side \\
\hline
\end{tabular}


The wrist markers are placed at the ends of a bar attached symmetrically with a wristband on the posterior of the wrist, as close to the wrist joint center as possible.

\begin{tabular}{|l|l|l|}
\hline LFIN & Left fingers & $\begin{array}{l}\text { Actually placed on the dorsum of the hand just below the head of the } \\
\text { second metacarpal }\end{array}$ \\
\hline
\end{tabular}

\section{Lower Body}

\section{Pelvis}

\begin{tabular}{|l|l|l|}
\hline LASI & Left ASIS & Placed directly over the left anterior superior iliac spine \\
\hline RASI & Right ASIS & Placed directly over the right anterior superior iliac spine \\
\hline
\end{tabular}

The above markers may need to be placed medially to the ASIS to get the marker to the correct position due to the curvature of the abdomen. In some patients, especially those who are obese, the markers either can't be placed exactly anterior to the ASIS, or are invisible in this position to cameras. In these cases, move each marker laterally by an equal amount, along the ASIS-ASIS axis. The true inter-ASIS Distance must then be recorded and entered on the subject parameters form. These markers, together with the sacral marker or LPSI and RPSI markers, define the pelvic axes.

\begin{tabular}{|l|l|l|}
\hline LPSI & Left PSIS & Placed directly over the left posterior superior iliac spine \\
\hline RPSI & Right PSIS & Placed directly over the right posterior superior iliac spine \\
\hline
\end{tabular}

LPSI and RPSI markers are placed on the slight bony prominences that can be felt immediately below the dimples (sacro-iliac joints), at the point where the spine joins the pelvis.

\begin{tabular}{|l|l|l|}
\hline SACR & $\begin{array}{l}\text { Sacral wand } \\
\text { marker }\end{array}$ & $\begin{array}{l}\text { Placed on the skin mid-way between the posterior superior iliac spines } \\
\text { (PSIS). An alternative to LPSI and RPSI. }\end{array}$ \\
\hline
\end{tabular}

SACR may be used as an alternative to the LPSI and RPSI markers to overcome the problem of losing visibility of the sacral marker (if this occurs), the standard marker kit contains a base plate and selection of short "sticks" or "wands" to allow the marker to be extended away from the body, if necessary. In this case it must be positioned to lie in the plane formed by the ASIS and PSIS points.

\section{Leg Markers}

\begin{tabular}{|l|l|l|}
\hline LKNE & Left knee & Placed on the lateral epicondyle of the left knee \\
\hline
\end{tabular}

To locate the "precise" point for the knee marker placement, passively flex and extend the knee a little while watching the skin surface on the lateral aspect of the knee joint. Identify where knee joint axis passes through the lateral side of the knee by finding the lateral skin surface that comes closest to remaining fixed in the thigh. This landmark should also be the point about which the lower leg appears to rotate. Mark this point with a pen. With an adult patient standing, this pen mark should be about $1.5 \mathrm{~cm}$ above the joint line, mid-way between the front and back of the joint. Attach the marker at this point. 


\begin{tabular}{|l|l|l|}
\hline LTHI & Left thigh & $\begin{array}{l}\text { Place the marker over the lower lateral 1/3 surface of the thigh, just } \\
\text { below the swing of the hand, although the height is not critical. }\end{array}$ \\
\hline
\end{tabular}

The thigh markers are used to calculate the knee flexion axis location and orientation. Place the marker over the lower lateral 1/3 surface of the thigh, just below the swing of the hand, although the height is not critical. The antero-posterior placement of the marker is critical for correct alignment of the knee flexion axis. Try to keep the thigh marker off the belly of the muscle, but place the thigh marker at least two marker diameters proximal of the knee marker. Adjust the position of the marker so that it is aligned in the plane that contains the hip and knee joint centers and the knee flexion/extension axis. There is also another method that uses a mirror to align this marker, allowing the operator to better judge the positioning.

\begin{tabular}{|l|l|l|}
\hline LANK & Left ankle & $\begin{array}{l}\text { Placed on the lateral malleolus along an imaginary line that passes } \\
\text { through the transmalleolar axis }\end{array}$ \\
\hline LTIB & $\begin{array}{l}\text { Left tibial } \\
\text { wand } \\
\text { marker }\end{array}$ & $\begin{array}{l}\text { Similar to the thigh markers, these are placed over the lower } 1 / 3 \text { of the } \\
\text { shank to determine the alignment of the ankle flexion axis }\end{array}$ \\
\hline
\end{tabular}

The tibial marker should lie in the plane that contains the knee and ankle joint centers and the ankle flexion/extension axis. In a normal subject the ankle joint axis, between the medial and lateral malleoli, is externally rotated by between 5 and 15 degrees with respect to the knee flexion axis. The placements of the shank markers should reflect this.

\section{Foot Markers}

\begin{tabular}{|l|l|l|}
\hline LTOE & Left toe & $\begin{array}{l}\text { Placed over the second metatarsal head, on the mid-foot side of the } \\
\text { equinus break between fore-foot and mid-foot }\end{array}$ \\
\hline LHEE & Left heel & $\begin{array}{l}\text { Placed on the calcaneous at the same height above the plantar surface } \\
\text { of the foot as the toe marker }\end{array}$ \\
\hline
\end{tabular}


APPENDIX E. Plug-in-Gait subject measurements

Subject Measurements

Mass (required): This is the mass of the subject in kilograms. $(1 \mathrm{lb}=2.2 \mathrm{~kg})$

Height (required): This is the height of the subject in millimeters. $(1 \mathrm{inch}=2.54 \mathrm{~cm})$

Inter-ASIS distance (optional): If this is not entered the model will calculate this distance based on the position of the LASI and RASI markers (recommended). If you are collecting data on an obese patient and can not properly place the ASIS markers, place those markers laterally and preserve the level of the ASIS. Palpate the LASI and RASI points and manually measure this distance. This measurement should be entered here for this scenario.

Head Angle (Calculated): This is the absolute angle of the head with the global coordinate system.

Leg Length (required): This is the true leg length measurement. It is measured from the ASIS to the medial malleolus. In the case of a patient who cannot straighten their legs, the measurement should be taken in two pieces. The leg length will be the sum of the length from the ASIS to the knee and from the knee to the medial malleolus.

Knee Width (required): This is the measurement of the knee width, about the flexion axis, in centimetres.

Ankle Width (required): This is the measurement of the ankle width, about the medial and lateral malleoli, in centimetres.

ASIS-Trochanter Distance (optional): This is the perpendicular distance from the trochanter to the ASIS point. If this value is not entered, then a regression formula is used to calculate the hip joint centre. This value will be calculated as part of this process. If this value is entered, it will be factored into an equation which represents the hip joint centre. For more details on this, please refer to the paper by Davis, et. al in the reference section. It is recommended that this value not be entered when processing the model.

Tibial Torsion (optional): Tibial torsion is the angle between the ankle flexion axis and the knee flexion axis. The sign convention is that if a negative value of tibial torsion is entered, the ankle flexion axis is rotated externally with respect to the knee flexion axis. If tibial torsion is entered while using a KAD, the ankle flexion/extension axis will be adjusted from the KAD's defined position to a position dictated by the tibial torsion value.

Thigh Rotation Offset (calculated): When a KAD is used, this value is calculated to account for the position of the thigh wand (marker). By using the KAD, placement of the thigh wand in the plane of the hip joint centre and the knee joint centre is not crucial. Please note that if you do not use a KAD, this value will be reported as zero because the model is assuming that the thigh wand has been placed exactly in the plane of the hip joint centre and the knee joint centre.

Shank Rotation Offset (calculated): The shank rotation offset is similar to the thigh rotation offset. This value is calculated if a KAD is present and removes the importance of placing the shank wand in the exact plane of the knee joint centre and the ankle joint centre. As above, if you do not use a KAD, then these values will be zero. 
${ }^{*}$ A note about the foot: VCM previously treated the foot as a vector, using only two markers during dynamic trials. Plug in Gait will process the foot as a vector if the heel marker is missing during dynamic trials. If all three markers are present then Plug in Gait will treat the foot as a three dimensional segment and NOT as a vector. The following parameters are used only if the foot is treated as a vector.

Foot Plantar Flexion Offset (calculated): Calculated as a rotation about the ankle flexion axis. This angle is measured between the line joining the heel and toe markers and the line joining the heel marker and the toe marker. This is one of the rotations performed in establishing the foot vector.

Foot Rotation Offset (calculated): This is a rotation about the foot rotation axis, which is perpendicular to the foot vector (after applying the foot plantar flexion offset) and the ankle flexion axis. This angle is measured between the line joining the heel and the toe markers and the line joining the ankle joint centre and the toe marker. This is the final rotation performed in establishing the orientation of the foot vector.

Shoulder Offset (required): This is the vertical distance from the centre of the glenohumeral joint to the marker on the acromion calivicular joint. Some researchers have used the (anterior/posterior girth)/2 to establish a guideline for the parameter.

Elbow Width (required): This is the distance between the medial and lateral epicondyles of the humerus.

Wrist Width (required): This is the distance between the ulnar and radial styloids.

Hand Thickness (required): This is the distance between the dorsal and palmar surfaces of the hand.

On a normal monitor the required, optional, and calculated fields will all have different coloured backgrounds. Some laptops may not display these colour differences.

Required fields will have a yellow hue background, optional fields will be white, and fields that are calculated by the model will be gray. 


\section{APPENDIX F. Anthropometric and demographic data of subjects}

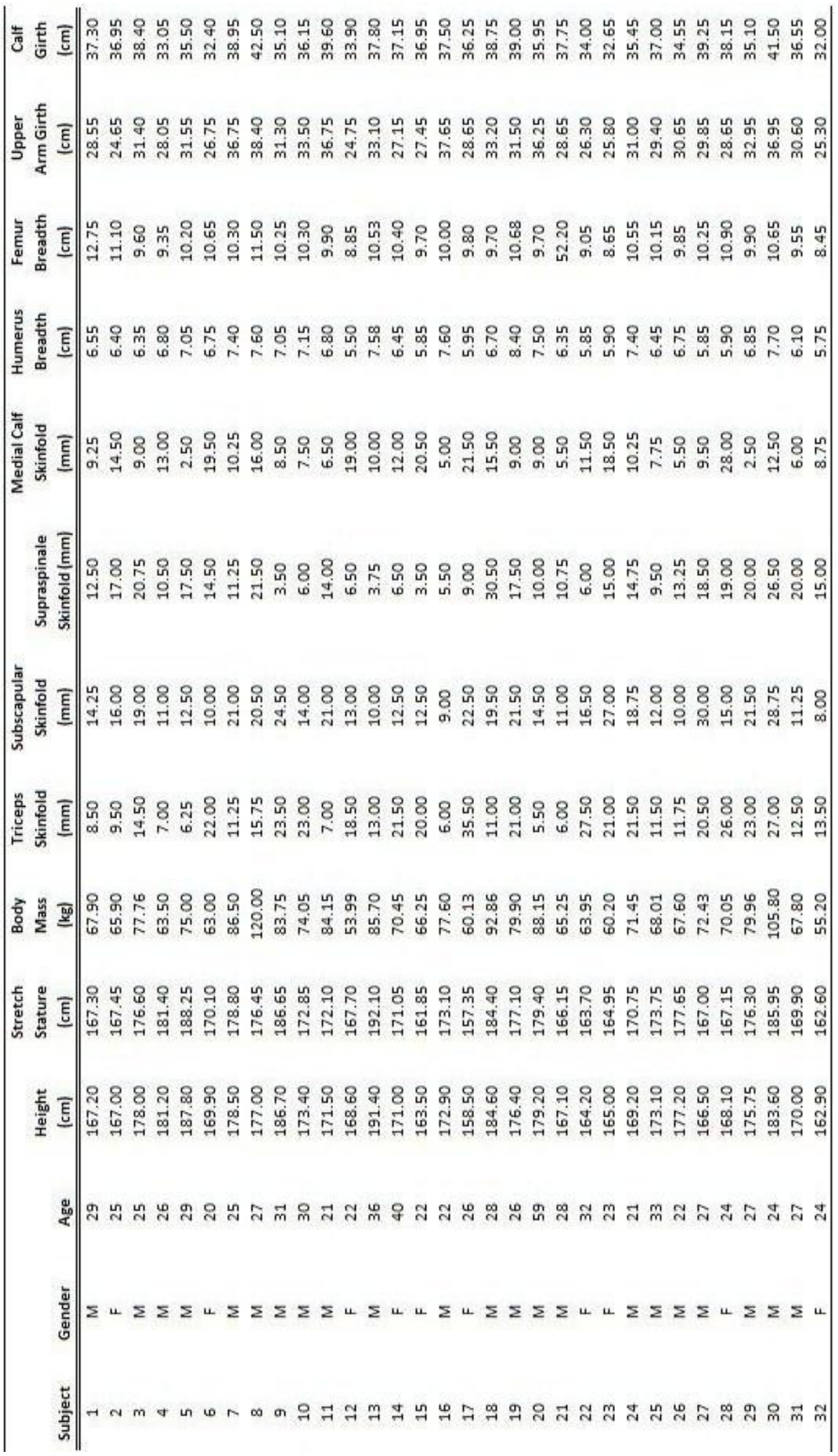


APPENDIX G. Calculated subject somatotypes

\begin{tabular}{|c|c|c|c|c|c|c|c|}
\hline Subject & Gender & Age & Endomorphy & Mesomorphy & Ectomorphy & HWR & BMI \\
\hline 1 & $M$ & 29 & 3.67 & 6.93 & 1.44 & 41.01 & 24.32 \\
\hline 2 & $\mathrm{~F}$ & 25 & 4.39 & 4.90 & 1.77 & 41.46 & 23.63 \\
\hline 3 & M & 25 & 5.21 & 4.25 & 1.71 & 41.38 & 24.54 \\
\hline 4 & M & 26 & 2.70 & 2.44 & 4.70 & 45.47 & 19.34 \\
\hline 5 & M & 29 & 3.36 & 3.51 & 4.10 & 44.64 & 21.27 \\
\hline 6 & $\mathrm{~F}$ & 20 & 4.70 & 3.93 & 2.71 & 42.75 & 21.82 \\
\hline 7 & M & 25 & 4.22 & 6.42 & 1.09 & 40.43 & 27.31 \\
\hline 8 & M & 27 & 5.50 & 8.33 & 0.10 & 35.77 & 38.30 \\
\hline 9 & M & 31 & 4.74 & 3.21 & 2.65 & 42.66 & 24.10 \\
\hline 10 & M & 30 & 4.31 & 5.75 & 1.55 & 41.16 & 24.71 \\
\hline 11 & M & 21 & 4.24 & 6.79 & 0.55 & 39.27 & 28.63 \\
\hline 12 & $\mathrm{~F}$ & 22 & 3.95 & 2.03 & 3.90 & 44.37 & 18.99 \\
\hline 13 & M & 36 & 2.36 & 4.06 & 3.31 & 43.57 & 23.34 \\
\hline 14 & $\mathrm{~F}$ & 40 & 4.12 & 4.36 & 1.74 & 41.42 & 24.01 \\
\hline 15 & $\mathrm{~F}$ & 22 & 3.88 & 4.55 & 0.89 & 40.00 & 24.69 \\
\hline 16 & $M$ & 22 & 1.94 & 7.28 & 1.16 & 40.58 & 25.76 \\
\hline 17 & $\mathrm{~F}$ & 26 & 6.76 & 5.09 & 0.97 & 40.16 & 23.93 \\
\hline 18 & $M$ & 28 & 5.55 & 3.95 & 1.22 & 40.72 & 27.25 \\
\hline 19 & M & 26 & 5.66 & 6.58 & 1.52 & 41.12 & 25.65 \\
\hline 20 & M & 59 & 2.89 & 5.62 & 1.03 & 40.31 & 27.47 \\
\hline 21 & M & 28 & 2.89 & 30.82 & 1.63 & 41.27 & 23.28 \\
\hline 22 & $\mathrm{~F}$ & 32 & 5.18 & 3.23 & 1.38 & 40.94 & 23.59 \\
\hline 23 & $\mathrm{~F}$ & 23 & 6.22 & 2.57 & 2.23 & 42.09 & 22.11 \\
\hline 24 & $M$ & 21 & 5.42 & 5.79 & 1.54 & 41.15 & 24.97 \\
\hline 25 & M & 33 & 3.31 & 4.52 & 2.58 & 42.57 & 22.68 \\
\hline 26 & M & 22 & 3.44 & 3.95 & 3.34 & 43.61 & 21.37 \\
\hline 27 & M & 27 & 6.61 & 5.20 & 0.92 & 40.06 & 26.15 \\
\hline 28 & $\mathrm{~F}$ & 24 & 5.93 & 4.81 & 1.14 & 40.55 & 24.81 \\
\hline 29 & M & 27 & 6.02 & 4.60 & 1.38 & 40.92 & 25.89 \\
\hline 30 & M & 24 & 6.95 & 6.07 & 0.57 & 39.32 & 31.39 \\
\hline 31 & M & 27 & 4.45 & 4.52 & 1.92 & 41.67 & 23.46 \\
\hline 32 & $\mathrm{~F}$ & 24 & 3.91 & 2.73 & 2.68 & 42.70 & 20.80 \\
\hline
\end{tabular}

John $\mathrm{H}$. Virginia University Libraries, 